BULLETIN (New Series) OF THE

AMERICAN MATHEMATICAL SOCIETY

Volume 35, Number 1, January 1998, Pages 1-56

S 0273-0979(98)00737-X

\title{
SYMBOLIC DYNAMICS AND MARKOV PARTITIONS
}

\author{
ROY L. ADLER
}

\begin{abstract}
The decimal expansion of real numbers, familiar to us all, has a dramatic generalization to representation of dynamical system orbits by symbolic sequences. The natural way to associate a symbolic sequence with an orbit is to track its history through a partition. But in order to get a useful symbolism, one needs to construct a partition with special properties. In this work we develop a general theory of representing dynamical systems by symbolic systems by means of so-called Markov partitions. We apply the results to one of the more tractable examples: namely, hyperbolic automorphisms of the two dimensional torus. While there are some results in higher dimensions, this area remains a fertile one for research.
\end{abstract}

\section{INTRODUCTION}

We address the question: how and to what extent can a dynamical system be represented by a symbolic one? The first use of infinite sequences of symbols to describe orbits is attributed to a nineteenth century work of Hadamard [H]. However the present work is rooted in something very much older and familiar to us all: namely, the representation of real numbers by infinite binary expansions. As the example of Section 3.2 shows, such a representation is related to the behavior of a special partition under the action of a map. Partitions of this sort have been linked to the name Markov because of their connection to discrete time Markov processes. However, as we shall see there is a purely topological version of the probabilistic (measure theoretic) idea. Markov partitions (topological ones), though not mentioned as such, are implicit in the invariant Cantor sets of the diffeomorphisms of the sphere constructed by Smale [Sm], the simplest one of which is the famous horseshoe map (see section 2.5, also [Sm] and [S, page 23]). Berg in his Ph.D. thesis $[\mathrm{Be}]$ was the first to discover a Markov partition of a smooth domain under the action of a smooth invertible map: namely, he constructed Markov partitions for hyperbolic automorphisms acting on the two dimensional torus. Markov partitions have come to play a pervasive role in understanding the dynamics of general hyperbolic systems-e.g., Anosov diffeomorphisms, axiom A diffeomorphisms, and psuedo-Anosov diffeomorphisms. Sinai [Si1], [Si2] constructed Markov partitions for Anosov diffeomorphisms (a simpler treatment can be found in Bowen [Bo3]). This class of maps includes hyperbolic automorphisms of $n$-dimensional tori, $n \geq 2$. Bowen [Bo1], [Bo2] went further and constructed Markov partitions for Axiom A diffeomorphisms. The pseudo-Anosov were introduced by Thurston [T], and Shub and Fathi $[\mathrm{FS}]$ constructed Markov partitions for them.

Received by the editors July 8, 1997.

1991 Mathematics Subject Classification. Primary 58F03, 58F08, 34 C35.

Appeared as MSRI Preprint No. 1996-053. 
Berg and Sinai carried their work out within the framework of measure theory. The purely topological approach first appears in the work of Bowen.

Shortly after the discovery of Berg, B. Weiss and the author [AW1], [AW2] proved that hyperbolic automorphisms of the two torus are measure theoretically isomorphic if and only if they have the same entropy. Their proof was based on two ideas:

1. symbolic representations of dynamical systems by means of Markov partitions,

2. coding between symbolic systems having equal entropy.

Each of these two aspects has undergone extensive development since. The present work is concerned with a systematic treatment of the first idea within the framework of point set topology: that is, we develop the notion of a discrete time topological Markov process without any recourse to measure theory and use it to obtain symbolic representations of dynamical systems. For comprehensive treatment of the second item we refer the reader to the book by Lind and Marcus [LM].

A disquieting aspect of the work of Adler-Weiss and similarly of Bowen's treatment of Markov partitions for toral automorphisms in [Bo2, page 12 ] is a certain vagueness where one expects certainty: namely, not quite knowing how to compute the numerical entries of certain integral matrices proven to exist. Theorem 8.4, the main one of Section 8, is an improvement on the results of Berg and Adler-Weiss and does not suffer from this difficulty. The proof involves four cases: Case I, the simplest, was done by Anthony Manning many years ago, as the author learned from Peter Walters, and still may be unpublished.

Another improvement in the present work over the literature is the dropping of requirements regarding size of elements in a Markov partition. In the present work these sets need not be small.

In Section 2, we briefly introduce the concept of an abstract dynamical system and then go on to give four important concrete examples of such systems: namely, multiplication maps, toral automorphisms, symbolic shifts, and the horseshoe map.

In Section 3, we discuss symbolic representations of dynamical systems and illustrate them for the concrete systems introduced in the previous section.

In Section 4, we present some general notions needed for our theory of symbolic representation of dynamical systems.

In Section 5, we introduce the notion of topological partition and show how one gets a symbolic representation from such an object. In order to simplify notation, an improvement in the choice of elements for such partitions was suggested by D. Lind: namely, replacing "proper sets with disjoint interiors", a proper set being one that is the closure of its interior, by "disjoint open sets whose closures cover the space". There is a difference between an open set and the interior of its closure, and exploiting this seemingly slight difference leads not only to notational conveniences but also to pleasant simplifications in subsequent proofs.

In Section 6, we define topological Markov partitions and prove Theorem 6.5, the main theorem of this work. This result concerns getting by means of Markov partitions the best that can be expected as far as symbolic representations of dynamical systems is concerned. Also in this section we prove a converse to the main theorem, Theorem 6.10 , by which one gets Markov partitions from symbolic representations. This leads to the question: does one construct Markov partitions to get symbolic representations, or does one produce symbolic representations to get 
Markov partitions? The answer to this riddle as far as the current evidence seems to indicate is discussed in Section 9.

In Section 7, we provide results useful for constructing Markov partitions, especially the final theorem of the section, Theorem 7.12 , which we apply in the next section. This theorem is adequate for the treatment of pseudo-Anosov diffeomorphisms by Shub and Fathi.

In Section 8, we construct certain special Markov partitions for arbitrary hyperbolic automorphisms of the two dimensional torus, which is the content of Theorem 8.4. These partitions have the virtue that a matrix specifying a hyperbolic automorphism is also the one that specifies a directed graph from which the symbolic representation is obtained. The proof we present, though involved, is quite elementary using mainly plane geometry.

In Section 9, we discuss some unsolved problems and future directions.

The spirit of this work is to rely solely on point set topology. We avoid any measure theory in this discussion. Perhaps a course in point set topology might be spiced up by using items in this work as exercises. In addition, our style of presentation is an attempt to accommodate students as well as experts.

The research behind this work was carried out over many years, in different places, and with help from a number of colleagues, particularly Leopold Flatto and Bruce Kitchens. Work was done at the Watson Research Center, University of Warwick, and MSRI. Most of the research for Sections 5-7 was done in the MSRI 1992 program in Symbolic Dynamics.

\section{AbstraCt AND CONCREte DynAmiCAL Systems}

At its most simplistic and abstract a dynamical system is a mathematical structure capable of generating orbits which evolve in discrete time. A map of a space into itself will achieve this. Depending on one's purpose additional structure is imposed: ours requires some topology. Consequently, for us an abstract dynamical system is a pair $(X, \phi)$ where $X$ is a compact metric space with metric, say, $d(\cdot, \cdot)$ and $\phi$ is a continuous mapping of $X$ into itself. We shall refer to $X$ as the phase space of the dynamical system. The orbit of a point $\mathrm{p} \in X$ is defined to be the sequence $\left(\phi^{n} \mathrm{p}\right)_{n=0,1,2, \ldots}$. We shall consider systems where $\phi$ is onto. Also we shall be mainly, though not exclusively, interested in invertible maps-i.e. where $\phi$ is a homeomorphism-in which case the orbit of a point $\mathrm{p} \in X$ is defined to be the bilaterally infinite sequence $\left(\phi^{n} \mathrm{p}\right)_{n \in \mathbb{Z}}$. For invertible maps we can speak of past, present, or future points of an orbit depending on whether $n$ is negative, zero or positive, while for non-invertible maps there is only the present and future.

For the above category of abstract systems, we have the following notion of total topological equivalence.

Definition 2.1. Two systems $(X, \phi),(Y, \psi)$ are said to be topologically conjugate, $(X, \phi) \simeq(Y, \psi)$, if there is a homeomorphism $\theta$ of $X$ onto $Y$ which commutes with $\phi$ and $\psi$ : i.e., $\phi \theta=\theta \psi$.

We introduce some classical concrete dynamical systems. The first type is most elementary. Though non-invertible, it illustrates admirably some essential ideas which we shall discuss later. 
ROY L. ADLER

2.1 Multiplication maps. Let $(X, f)$ be the system whose phase space is the complex numbers of modulus one-i.e. elements of the unit circle-acted upon by the mapping $f: z \rightarrow z^{n}$ for some integer $n>1$.

For our purposes it is more convenient to consider a topologically and algebraically equivalent formulation. Let $X=\mathbb{R} / \mathbb{Z}$ where $\mathbb{R}$ is the real line and $\mathbb{Z}$ the subgroup of integers. Recall that elements in $X$ are cosets modulo $\mathbb{Z}$. The coset of $x \in \mathbb{R}$ modulo $\mathbb{Z}$, which we denote by $\{x\}$, is the set $\{x+z \mid z \in \mathbb{Z}\}$ of lattice translates of $x$. Invoking some standard terminology, the real line $\mathbb{R}$ can be referred to as the universal cover of the the circle $X$. In view of the fact that $\mathbb{Z}$ acts as a group of transformations on the universal cover $\mathbb{R}$, a coset is also called a $\mathbb{Z}$-orbit. Two points $x, x^{\prime}$ in the same coset or $\mathbb{Z}$-orbit are said to be equivalent $\bmod \mathbb{Z}$. The metric is given by defining the distance between pairs of cosets as the smallest Euclidean distance between pairs of members. Recall that the coset of $x+y$ depends only on the coset of $x$ and that of $y$ : that is, if $x^{\prime} \in\{x\}$ and $y^{\prime} \in\{y\}$, then $\left\{x^{\prime}+y^{\prime}\right\}=\{x+y\}$. Thus addition of cosets given by $\{x\}+\{y\} \equiv\{x+y\}$ is well defined and so is the multiplication-by-n map $f:\{x\} \mapsto\{x\}+\cdots+\{x\}$ (n-times) $=\{n x\}$. This map is continuous with respect to the metric. It is not invertible: every coset $\{x\}$ has $n$ pre-images which are $\{(x+m) / n\}, m=1, \ldots, n$.

The closed unit interval $[0,1]$ is a set referred to as a fundamental region for the action of $\mathbb{Z}$ on $\mathbb{R}$.

Definition 2.1.1. A fundamental region is defined as a closed set such that

1. it is the closure of its interior;

2. every orbit under the action has at least one member in it (this is equivalent to the statement that the translates of the unit interval by elements of $\mathbb{Z}$ tile $\mathbb{R})$;

3. no point in the interior is in the same $\mathbb{Z}$-orbit as another one in the closed region (this restriction does not apply to two boundary points- e.g. the points 0 and 1 are in the same one).

Fundamental regions are not unique: for example, the interval $[1,2]$ is also a fundamental region for the action of $\mathbb{Z}$ on $\mathbb{R}$, though not a particularly useful one.

One can give another equivalent reformulation of the phase space of these systems in terms of a fundamental region with boundary points identified. Let $X$ be the closed unit interval with 0 identified with 1 . We define a metric on $X$ by

$$
d(x, y)=\min (|x-y|,|x-y-1|,|x-y+1|) .
$$

From now on let us take the notation $\{x\}$ to mean the fractional part of a real number $x$. On $X$ the map $f$ takes the form

$$
f(x)=\{n x\}
$$

Since all numbers in a coset have the same fractional part and that number is the unique member of the intersection of the coset and $X$, this new interpretation of $\{x\}$ is consistent with the old.

More serious examples are continuous automorphisms of certain compact Abelian groups-namely, the n-dimensional tori. For simplicity we restrict the discussion to the case of dimension two, generalization to higher dimensions being quite analogous. 
2.2 Toral automorphisms. Consider the two dimensional torus $\mathbb{R}^{2} / \mathbb{Z}^{2}$ and a continuous group automorphism $\phi$. Here the universal cover of the 2 -torus is $\mathbb{R}^{2}$. The description of the action of the integers on the real line generalizes in a straightforward manner to the action of the subgroup $\mathbb{Z}^{2}$ of points with integer coordinates on the universal cover $\mathbb{R}^{2}$. The definitions of cosets, lattice translates, orbits, addition, and the metric are quite similar.

A continuous automorphism $\phi$ is specified by a $2 \times 2$ matrix A : with integer entries and determinant \pm 1 . Let

$$
\mathrm{A}=\left(\begin{array}{ll}
a & b \\
c & d
\end{array}\right)
$$

The matrix A determines an invertible linear transformation on $\mathbb{R}^{2}$. We represent the points in the plane by row vectors and the action of the linear transformation by right ${ }^{1}$ matrix multiplication. The map $\phi$ is then defined as follows: the image of the coset containing $(x, y)$ is the one containing $(a x+c y, b x+d y)$. This map is well-defined-i.e., the image does not depend on the choice of coset representative $(x, y)$ - because $\mathrm{A}$ is invertible and maps $\mathbb{Z}^{2}$ onto itself.

Some things to note. The map $\phi$ is a homeomorphism. The coset $\{0\}=\mathbb{Z}^{2}$ is a fixed point of $\phi$. There may of course be other fixed points. A pair $(x, y)$ is in a coset which is a fixed point if and only if it satisfies

$$
(x, y) \mathrm{A}=(x, y)+(m, n)
$$

for some pair of integers $(m, n)$. The only solutions are rational. In addition, a coset is periodic under $\phi$ if and only if it is fixed under some iterate $\mathrm{A}^{n}$. Furthermore, if a coset contains a point with rational coordinates, then it is periodic, which follows from the fact that the product of the denominators in a rational pair $(x, y)$ bounds the denominators in the sequence $(x, y),(x, y) \mathrm{A},(x, y) \mathrm{A}^{2}, \ldots$, which implies that the orbit of $(x, y)$ is finite.

The plane $\mathbb{R}^{2}$ is the universal cover of the 2-torus, and any closed unit square with sides parallel to the axes is a fundamental region. We shall call the one with its lower left corner at the origin the principal fundamental region. Like the one dimensional case, we can formulate the system in terms of it. Let the phase space $X$ be the closed unit square with each point on one side identified with its opposite on the other. The coset of $(x, y)$ intersects $X$ in a unique point: namely, $(\{x\},\{y\})$. On $X$ the map $\phi$ takes the form

$$
\phi(x, y)=(\{a x+c y\},\{b x+d y\}) .
$$

Unlike the case of one dimension, other fundamental regions, as we shall see, play a crucial role in studying the action of automorphisms.

Finally we come to the most basic of concrete systems. Ultimately we shall show to what extent they model others, in particular multiplication maps and hyperbolic toral automorphisms.

2.3 Symbolic shifts. Let $\mathcal{A}$, called an alphabet, denote an ordered set of $N$ symbols, often taken to be $\{0,1, \ldots, N-1\}$. The phase space of this system is the space

$$
\Sigma_{N}=\mathcal{A}^{\mathbb{Z}}=\left\{s=\left(s_{n}\right)_{n \in \mathbb{Z}} \mid s_{n} \in \mathcal{A}\right\}
$$

\footnotetext{
${ }^{1}$ Right multiplication on row vectors turns out to be a little more convenient than left multiplication on column vectors.
} 


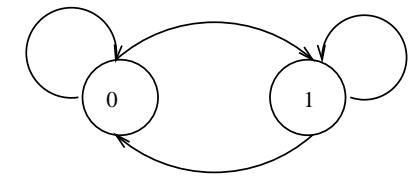

Vertex labelled

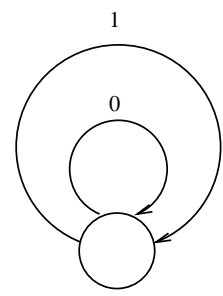

Edge labelled

Figure 1

of all bi-infinite sequences of elements from a set of $\mathrm{N}$ symbols. One can think of an element of this space as a bi-infinite walk on the complete directed graph of $N$ vertices which are distinctly labelled. Sometimes it is more convenient to label edges, in which case the picture is a single node with $N$ oriented distinctly labelled loops over which to walk. In Figure 1 we have depicted the graph for $\Sigma_{2}$ by both methods of labelling.

The shift transformation $\sigma$ is defined by shifting each bi-infinite sequence one step to the left. This is expressed by

$$
(\sigma s)_{n}=s_{n+1} .
$$

We define the distance $d(s, t)$ between two distinct sequences $s$ and $t$ as $1 /(|n|+1)$ where $\mathrm{n}$ is the coordinate of smallest absolute value where they differ. Thus if $d(s, t)<1 / n$ for $n>0$, then $s_{k}=t_{k}$ for $-n<k<n$. This metric makes the space $\Sigma_{N}$ one of the important compact ones-namely, the Cantor discontinuum- and the shift a homeomorphism. The symbolic system $\left(\Sigma_{N}, \sigma\right)$ is called the full $N$-shift.

Restricting the shift transformation of a full shift $\Sigma_{N}$ to a closed shift-invariant subspace $\Sigma$, we get a very general kind of dynamical system $(\Sigma, \sigma)$ called a subshift. Given a symbolic sequence $s=\left(s_{n}\right)_{n \in \mathbb{Z}}$ and integers $m<n$, we shall use the notation $s_{[m, n]}$ to stand for the $m-n+1$-tuple $\left(s_{m}, s_{m+1}, \ldots, s_{n}\right)$. Given a symbolic phase space $\Sigma$, we call a k-tuple an allowable $k$-block if it equals $s_{[m, m+k-1]}$ for some $s \in \Sigma$.

Returning to the realm of the more specific from our momentary excursion into the less knowable, we define shift of finite type, also called topological Markov shift, as the subshift of a full shift restricted to the set $\Sigma_{G}$ of bi-infinite paths in a finite directed graph $G$ derived from a complete one by possibly removing some edges.

Usually we denote the space $\Sigma_{G}$ by $\Sigma_{\mathrm{A}}$ where A is a matix of non-negative integers $a_{i j}$ denoting the number of edges leading from the $i$-th node to the $j$-th. The term "Markov" is derived from the resemblance to Markov chains for which the $a_{i j}$ are probabilities instead of integers. One thing to note is that the $i j$ entry of $\mathrm{A}^{n}$ is the number of paths of length $n$ beginning at $i$-th node and ending at the $j$-th. Often however $\mathrm{A}$ is an $N \times N$ matrix of zeroes and ones specifying a directed graph of $N$ nodes (edges) according to the following: the $i$-th node (edge) is connected to the $j$-th, $i \longrightarrow j$, if and only if $a_{i j}=1$. Whether dealing with nodes or edges, we call A a transition matrix and restate for zero-one matrices the above 


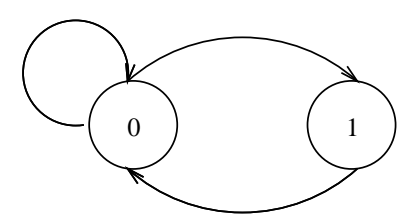

FIGURE 2. Fibonacci shift

definition by

$$
\Sigma_{G}=\Sigma_{\mathrm{A}} \equiv\left\{s=\left(\ldots, s_{n}, \ldots\right) \mid a_{s_{n}, s_{n+1}}=1, s_{n} \in \mathcal{A}, n \in \mathbb{Z}\right\} .
$$

Remark. Let $\left(\Sigma_{G}, \sigma\right)$ be a topological shift given by a node-labelled directed graph $G$. Nodes from which there is no return are called transient, the rest recurrent. A node is transient if and only if either it has no predecessor nodes or all its predecessors are transient. This statement is not as circular as it seems: for the set of predecessors of any set of transient nodes, if non-empty, is a strictly smaller set of transient nodes. The only symbols which appear in bi-infinite sequences of $\Sigma_{G}$ are labels of recurrent nodes.

Figure 2 describes the Fibonacci or golden ratio shift, so-called because the number of admissible n-blocks (paths of length $n$ ) are the Fibonacci numbersnamely, there are two 1-blocks, three 2-blocks, five 3-blocks, ... .

Here the space $\Sigma_{\mathrm{A}}$ is given by the matrix

$$
\mathrm{A}=\left(\begin{array}{ll}
1 & 1 \\
1 & 0
\end{array}\right)
$$

If we label the first node by 0 and and the second by 1 , then only sequences of 0 's and 1's with 1's separated by 0's are admissible. While other shifts of finite type can be specified by graphs with either nodes or edges labelled, there is no edge-labelled graph for the Fibonacci shift.

Given a node-labelled graph $G$, we define the edge graph $G^{(2)}$ by labelling the edges. For labels we can use the allowable 2-blocks. In general we define the higher edge graphs $G^{(n)}$ as follows. The alphabet consists of all allowable blocks $\left[a_{1}, \ldots, a_{n}\right]$ gotten from paths of length $n$ on $G$. The transitions are defined by

$$
\left[a_{1}, \ldots, a_{n}\right] \longrightarrow\left[b_{1}, \ldots, b_{n}\right]
$$

if and only if $b_{1}=a_{2}, \ldots, b_{n-1}=a_{n}$.

There is a one-side version of the full $N$-shift: namely,

$$
\Sigma_{N}^{+}=\left\{s=\left(s_{0}, s_{1}, \ldots\right) \mid s_{n} \in \mathcal{A}, n=0,1,2, \ldots\right\} .
$$

On this space the shift transformation $\sigma$ is similarly defined: namely, $(\sigma s)_{n}=s_{n+1}$ but only for non-negative $n$. It acts by shifting sequences one step to the left and dropping the first symbol. The metric on this phase space is defined the same as before but absolute value signs are not needed. We also have one-side versions of shifts of finite type. In one-side symbolic systems, the shift transformation, like a multiplication map, is continuous but not invertible.

2.4 Horseshoe map. We present here a brief informal description of Smale's horseshoe map. For more details consult [Sm], [S, page 23], [HK, page 273]. 
ROY L. ADLER

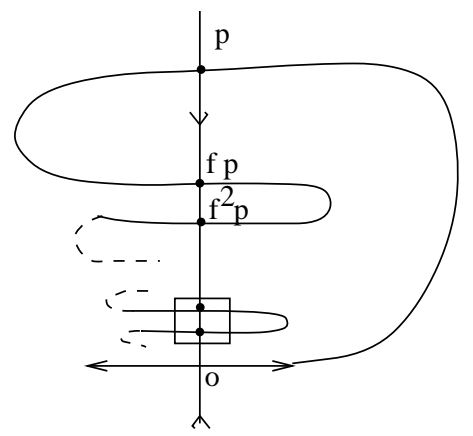

FiguRE 3. Hyperbolic fixed point with homoclinic point

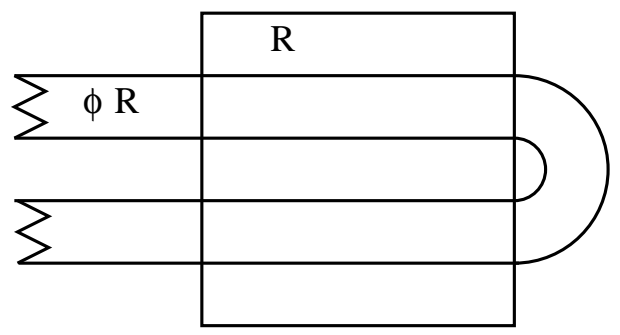

FiguRE 4. Horseshoe map

Let $f$ be a diffeomorphism of the plane with a hyperbolic fixed point at the origin. This means that there are two invariant curves through the origin such that iterates under $f$ of points on one and iterates under $f^{-1}$ of points on the other converge exponentially to the origin. The first is called the stable manifold and the second the unstable manifold. Let $p$ be a transverse homoclinic point-i.e. a point where the unstable manifold crosses the stable manifold. Then $f^{n} p$ will be a sequence of homoclinic points converging to the origin. See Figure 3. Somewhere near the origin there will be a rectangular neighborhood $R$ such that $\phi R$, where $\phi=f^{n}$ for some iterate of $f$ will intersect $R$ as in Figure 4 .

In Smale's work [Sm] more complicated diffeomorphisms than the simple horseshoe map are considered. These involve many fixed points where a stable manifold of one might cross unstable manifolds of others.

\section{Exercises.}

2.1 Prove that the canonical map $\psi: G^{(n)} \longrightarrow G$ defined by $\psi\left[s_{1}, \ldots, s_{n}\right]=s_{1}$ gives topological conjugacy of $\left(\Sigma_{G^{(n)}}, \sigma\right)$ and $\left(\Sigma_{G}, \sigma\right)$.

\section{Symbolic RePRESEntations}

Shifts of finite type contain a great deal of complexity, yet are the best understood dynamical systems. Such symbolic dynamical systems can be used to analyze general discrete time ones. For example, a good symbolic representation will show how to identify periodic orbits, almost periodic ones, dense ones, etc.

Representing a general dynamical system by a symbolic one involves a fundamental complication. We have two desires: we would like a continuous one-to-one 


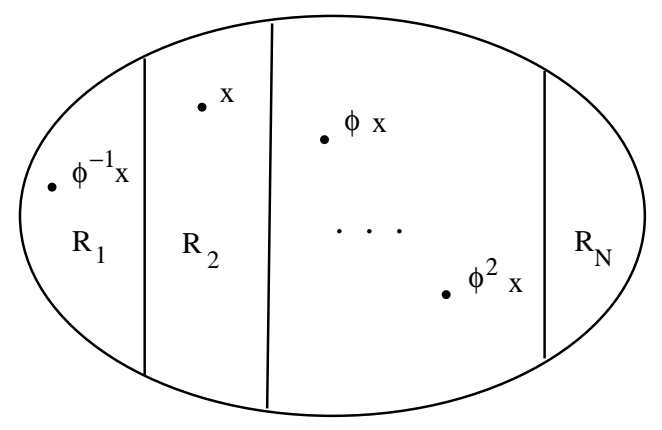

Figure 5. Partitioning a dynamical system

correspondence between orbits $\phi^{n} x$ of the first and orbits $\sigma^{n} s$ of the second, and we want the shift system to be one of finite type. Unfortunately, these two desires are usually in conflict: constraints placed by topology must be observed. On one hand a continuous one-to-one correspondence makes $X$ homeomorphic to a shift system. On the other hand a shift system is totally disconnected while $X$ is often a smooth manifold. Thus for the most part we must abandon the quest of finding a topological conjugacy between a given dynamical system and a shift of finite type. However, we shall see that by sacrificing one-to-one correspondence we can still salvage a satisfactory symbolization of orbits. We are reminded of arithmetic in which we represent real numbers symbolically by decimal expansions, unique for the most part, but must allow two expansions for certain rationals. To do otherwise would just make the instructions for arithmetical operations unnecessarily complicated. The most natural way to associate a symbolic sequence with a point in a dynamical system is to track its history as illustrated in Figure 5 through a family of sets indexed by an alphabet of symbols.

This is easy, but what is more difficult is to get a family for which each history represents just one point. It is no achievement to specify a family for which each history might represent more than one point. However, we must live with the inevitability that each point might have more than one associated history. Having found a family of sets, the orbits through which determine a unique point, we want still more: namely, we would like the totality of sequences which arise to comprise a subshift of finite type. In order to do this, we must find a family with special properties. We shall look at some examples for guidance as to what these properties ought to be, and families of sets possessing them will be called Markov partitions.

The first example is the trivial case of a dynamical system which is identical with its symbolic representation: namely, a topological Markov shift.

3.1 Cylinder set partition for symbolic sequences. Let $\left(\Sigma_{\mathrm{A}}, \sigma\right)$ be a topological Markov shift, vertex labelled by an alphabet $\mathcal{A}$. We form the partition $\mathcal{C}=\left\{C_{a}: a \in \mathcal{A}\right\}$ of elementary cylinder sets determined by fixing the 0 -th coordinate: i.e., $C_{a}=\left\{s \in \Sigma_{A}: s_{0}=a\right\}$. Tracking the history of an orbit of an element $s \in \Sigma_{A}$ through this partition means getting a sequence $\left(s_{n}\right)_{n \in \mathbb{Z}}$ such that $\sigma^{n} s \in C_{s_{n}}$. But this sequence is $s$ itself. Let us point out the salient features of this partition. 
First $^{2}$,

$$
\bigcap_{n=0}^{\infty} \bigcap_{-n}^{n} \phi^{-k} C_{s_{k}}=\{s\} .
$$

Second, if $s \in C_{a} \cap \sigma^{-1} C_{b} \neq \emptyset$, then $s \in C_{a}$ and $\sigma s \in C_{b}$ : i.e., $s_{0}=a, s_{1}=b$. In terms of the graph, this means there is an edge from $a$ to $b$. An absolutely obvious property of directed graphs is the following. If there is an edge from $a$ to $b$, and an edge from $b$ to $c$, then there is a path from $a$ to $c$ via $b$. This property can be reformulated as follows. If $C_{a} \cap \sigma^{-1} C_{b} \neq \emptyset$ and $C_{b} \cap \sigma^{-1} C_{c} \neq \emptyset$, then $C_{a} \cap \sigma^{-1} C_{b} \cap \sigma^{-2} C_{c} \neq \emptyset$. This property has a length $n$ version for arbitrary $n$ : namely, $n$ abutting edges form a path of length $n+1$, and this can be reformulated to read that $n$ pair-wise non-empty intersections lead to an $(n+1)$-fold non-empty intersection. We shall call such a countable set of conditions for $n=2,3, \ldots$ the Markov property: it turns out to be a key requirement in getting the desired symbolic representation from a partition.

Finally, there is another important feature of the partition $\mathcal{C}=\left\{C_{a}: a \in \mathcal{A}\right\}$ : namely, the sets of this partition have a product structure respected by the shift which is described as follows. Let $s \in C_{a}$-in other words, $s_{0}=a-$ and define two sets

$$
v_{a}(s) \equiv \bigcap_{0}^{\infty} \sigma^{-k} C_{s_{k}}
$$

which we shall call the vertical through $s$ and

$$
h_{a}(s) \equiv \bigcap_{-\infty}^{0} \sigma^{-k} C_{s_{k}}
$$

which we shall call the horizontal. A sequence $s \in C_{a}$ is the sole member of the intersection of its vertical and horizontal-i.e. $\{s\}=v_{a}(s) \cap h_{a}(s)$. Furthermore, for $s, t \in C_{a}$ there is a unique sequence in the intersection of the horizontal through $s$ and the vertical through $t$ : namely, $\left\{\left(\ldots s_{-2}, s_{-1}, s_{0}=t_{0}, t_{1}, t_{2}, \ldots\right)\right\}=v_{a}(s) \cap$ $h_{a}(t)$. We define a map of $C_{a} \times C_{a}$ onto $C_{a}$ by $(s, t) \mapsto h_{a}(s) \cap v_{a}(t)$, or rather the sole element of this intersection. It is easily verified that this map is continuous and its restriction to $h_{a}(s) \times v_{a}(t)$ for any $s, t \in C_{a}$ is a homeomorphism of $h_{a}(s) \times v_{a}(t)$ onto $C_{a}$. Finally $\sigma$ respects this product structure in the sense that if $s \in C_{a} \cap$ $\sigma^{-1} C_{b}$, then:

$$
\begin{aligned}
& \sigma v_{a}(s) \subset v_{b}(\sigma s), \\
& \sigma h_{a}(s) \supset h_{b}(\sigma s) .
\end{aligned}
$$

This last property is closely connected with the Markov one.

The next example is based on the binary expansions of real numbers and illustrates what one should expect of a good symbolic representation of a dynamical system.

\footnotetext{
${ }^{2}$ From now on we shall commit a convenient semantic error of confusing a set consisting of a single point with the point itself and so dispense with the surrounding braces.
} 


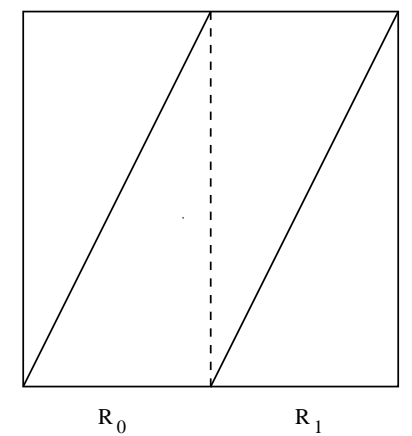

FiguRE 6 . Graph of multiplication by $2(\bmod 1)$

3.2 Symbolic representation for multiplication by two. Let $(X, f)$ be the multiplication system where and $f: x \rightarrow\{2 x\}$. See Figure 6 for the graph of $f$. Recall that the domain $\Sigma_{2}^{+}$of the one-sided full 2-shift dynamical system $\left(\Sigma_{[2]}^{+}, \sigma^{+}\right)$ is the set of one-sided infinite walks on the edge-labelled graph in Figure 1. We can equate a sequence $s=\left(s_{n}\right)_{n \in \mathbb{Z}}$ with the binary expansion $s_{1} s_{2} s_{3} \ldots$ Consider the map $\pi$ from $\Sigma_{[2]}^{+}$to $X$ defined by $\pi\left(s_{1}, s_{2}, \ldots\right)=\left\{s_{1} / 2+s_{2} / 4+\ldots\right\}$. It is readily verified that

(i) $f \pi=\pi \sigma^{+}$,

(ii) $\pi$ is continuous,

(iii) $\pi$ is onto,

(iv) there is a bound on the number of pre-images (in this case two), and

(v) there is a unique pre-image of "most" numbers (here those with binary expansions not ending in an infinite run of all zeros or all ones).

The map $\pi$ is not a homeomorphism, but we do have a satisfactory representation of the dynamical system by a one-sided 2-shift in the sense that: orbits are preserved, every point has at least one symbolic representative, there is a finite upper limit to the number of representatives of any point, and every symbolic sequence represents some point. This is a example of what is known as a factor map, which we shall formalize in Section 4.

As we have led the reader to expect, there is an alternate definition of $\pi$ in terms of a partition. Consider $\mathcal{R}=\left\{R_{0}=(0,1 / 2), R_{1}=(1 / 2,1)\right\}$. The elements of this family are disjoint open intervals whose closure covers the unit interval. The map $\pi$ which associates sequences with points has an alternate expression in terms of this family: namely,

$$
\pi\left(s_{1}, s_{2}, \ldots\right)=\bigcap_{n=0}^{\infty} \overline{R_{s_{1}} \cap f^{-1}\left(R_{s_{2}}\right) \cap \cdots \cap f^{-n}\left(R_{s_{n+1}}\right)} .
$$

Remark. The reader might wonder about defining $\pi$ by the simpler expression

$$
\pi\left(s_{1}, s_{2}, \ldots\right)=\bigcap_{n=0}^{\infty} \overline{f^{-n}\left(R_{s_{n+1}}\right)} .
$$

There are cases where this would suffice, but a difficulty can arise and does here. In $X$ the point 0 which is identified with 1 is a fixed point of $f$ which implies that 


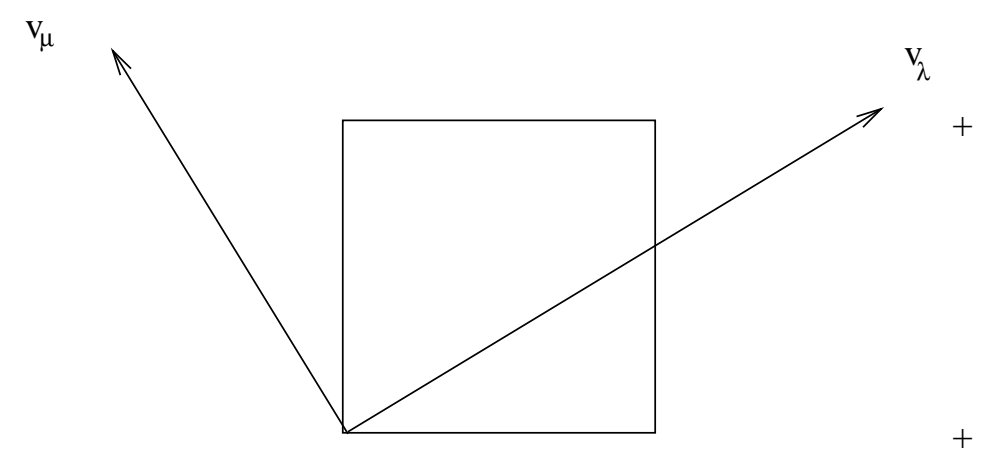

Figure 7. The torus and eigen-directions of $A$

$0 \in f^{-n} \overline{R_{i}}$ for $i=0,1$ and $n \geq 0$. Thus, except for the all 0 or all 1 sequence, $\pi\left(s_{1}, s_{2}, \ldots\right)$ is a set which does not consist of a singleton: it contains two real numbers, one of which is the fixed point 0 , and this renders $\pi$ ill-defined. The most we can say in general is that

$$
\bigcap_{n=0}^{\infty} \overline{R_{s_{1}} \cap f^{-1}\left(R_{s_{2}}\right) \cap \cdots \cap f^{-n}\left(R_{s_{n+1}}\right)} \subsetneq \bigcap_{n=0}^{\infty} \overline{f^{-n}\left(R_{s_{n+1}}\right)} .
$$

However, for the so-called expansive dynamical systems, when the size of partition elements is uniformly small enough, equality holds, in which case $\pi$ would be welldefined (see Proposition 5.8).

Next we consider hyperbolic automorphisms of the 2-torus. This was the first smooth class of invertible dynamical systems found to have Markov partitions. This discovery was made by K. Berg [Be] in 1966 in his doctoral research. A short time later R. Adler and B. Weiss [AW1] constructed some special Markov partitions in order to prove that two such systems are conjugate in the measure theoretic sense if they have the same entropy. For these systems topological conjugacy implies measure conjugacy, but not conversely. We shall give a formal development for the general two-dimensional case in a later chapter. Before making that plunge, we shall wet our toes with an informal discussion of one specific illustrative case. A rigorous proof of what we are about to describe will be achieved by Theorem 7.13.

3.3 Partition for a toral automorphism. Take the matrix

$$
\mathrm{A}=\left(\begin{array}{ll}
1 & 1 \\
1 & 0
\end{array}\right)
$$

which we have met before in quite a different context. Let the phase space $X$ be the two-torus and $\phi$ be given by $\mathrm{A}$ : that is,

$$
\phi(x, y)=(\{x+y\}, x) .
$$

The matrix A has two eigenvalues: $\lambda=(1+\sqrt{5}) / 2$ and $\mu=(1-\sqrt{5}) / 2$. Observe that $\lambda>1$ and $-1<\mu<0$. Associated with these eigenvalues are the eigenvectors $\mathrm{v}_{\lambda}$ pointing into the first quadrant and $\mathrm{v}_{\mu}$ into the second. In Figure 7 we have drawn two lines through the origin in the eigenvector directions. The action of $\mathrm{A}$ on a vector is to contract its $\mathbf{v}_{\mu}$-component by $|\mu|$ and expand its $\mathbf{v}_{\lambda}$-component by $\lambda$. Note $\mu$ is negative, which causes a direction reversal besides a contraction in 


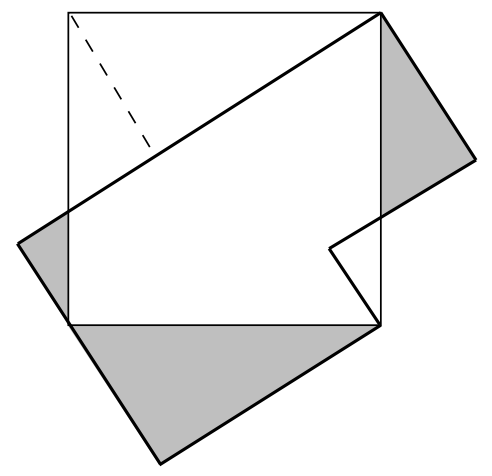

$+$

Figure 8. Another fundamental region of 2-torus

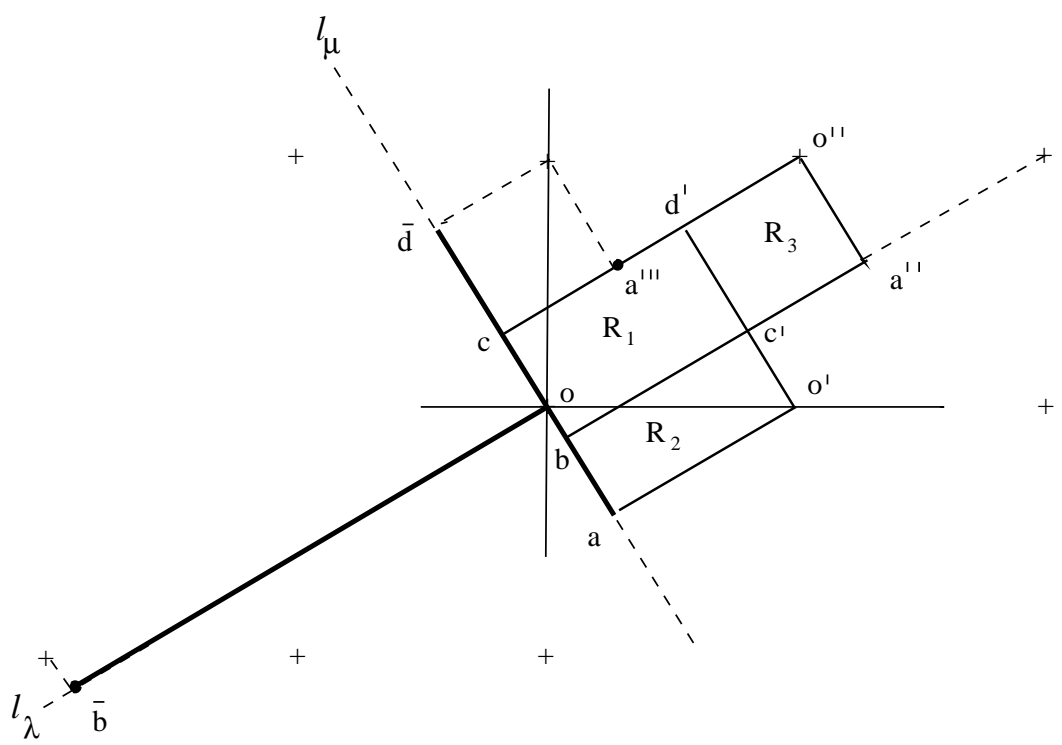

Figure 9. Partition of 2-torus

the $\mathrm{v}_{\mu}$-component. We refer to the direction of $\mathrm{v}_{\lambda}$ as the expanding direction and that of $\mathrm{v}_{\mu}$ as the contracting direction.

In Figure 8 we draw another region with sides parallel to the expanding and contracting directions. That it is a fundamental region is verified by noting that each of the three triangles sticking out of the unit square is a translation by an element of $\mathbb{Z}^{2}$ of one of the three missing triangles inside.

We call this fundamental region the principal one and draw within it the collection of open rectangles $\mathcal{R}=\left\{R_{i}: i=1,2,3\right\}$ as depicted in Figure 9 . This family is an example of a type of partition we shall later describe as Markov. We label significant points using the same letters for those which are equivalent.

The image of this partition under the linear transformation determined by A is depicted in Figure 10. In drawing it the following calculations come into play: $(1,0) \mathrm{A}=(1,1),(1,1) \mathrm{A}=(2,1),(2,1) \mathrm{A}=(3,2)$. 


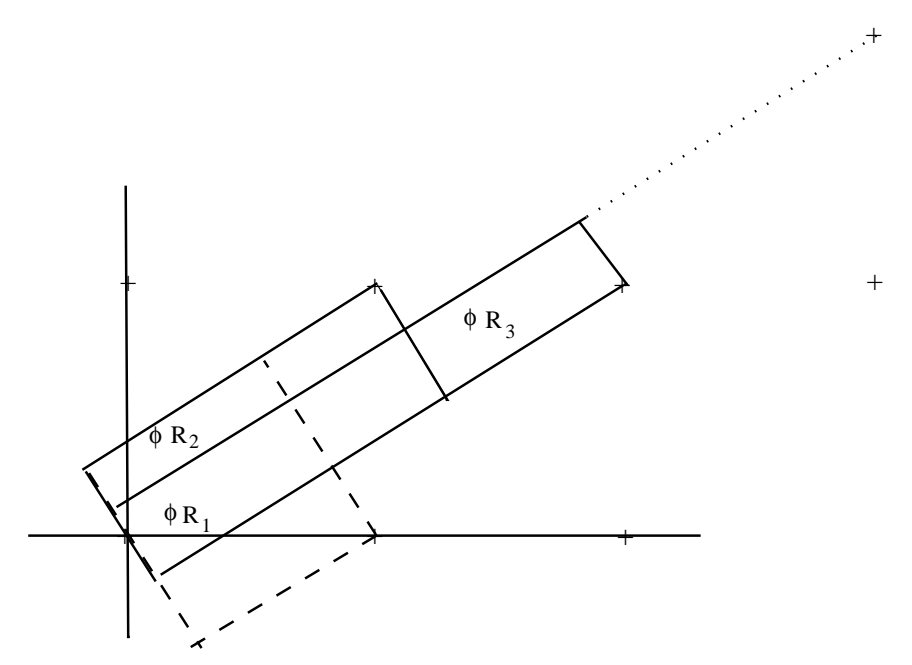

Figure 10. Its image

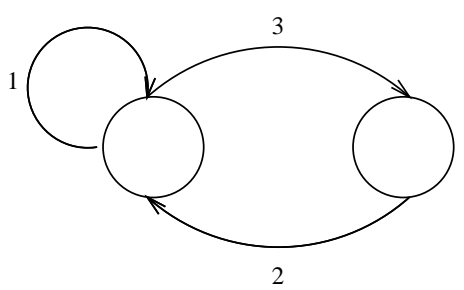

FiguRE 11. Edge graph for $\phi$ acting on $\mathcal{R}$

Along with the image we have included an outline of the original partition. Notice that $\phi R_{3}$ is actually the same as $R_{2}$. Also notice how the other $\phi R_{i}$ overlap $R_{1}$ and $R_{3}$. The manner in which the image partition intersects the original partition can be summed up as follows: $\phi R_{i} \cap R_{j} \neq \emptyset$ according to whether $j$ follows $i$ in the edge graph in Figure 11.

The boundary of the sets in $\mathcal{R}, \partial R_{i}=\overline{R_{i}}-R_{i}$, consists of various line segments in the $\mathrm{v}_{\lambda}$ and $\mathrm{v}_{\mu}$ directions. The union of those of the $R_{i}$ 's in the $\mathrm{v}_{\lambda}$-direction is called the expanding boundary of the partition and those in the $\mathrm{v}_{\mu}$-direction, the contracting boundary. By lattice translations of the various bounding segments, we can reassemble their union into two intersecting line segments through the origin, $\mathrm{o} \overline{\mathrm{b}}$ and $\mathrm{a} \overline{\mathrm{d}}$, as shown in Figure 10.

The behavior of the boundary under the action A leads to a topological Markov shift representation. The essential properties are that ad contains its image under $\mathrm{A}$; whereas ob is contained in its image, or equivalently ob contains its inverse image. Because A preserves eigen-directions and keeps the origin fixed, it is easy to see that ob gets stretched over itself; but because there is a reflection involved, it is not enough to know that the length of ad is contracted by A. We must show that the points a and $\overline{\mathrm{d}}$ on the line segment a $\overline{\mathrm{d}}$ have their images within that segment. These points are the projections to $l_{\mu}$ in the $\mathbf{v}_{\lambda}$-eigen-direction from $(0,1)$ and $(1,0)$ respectively: so their images are the projections from the images of these lattice 


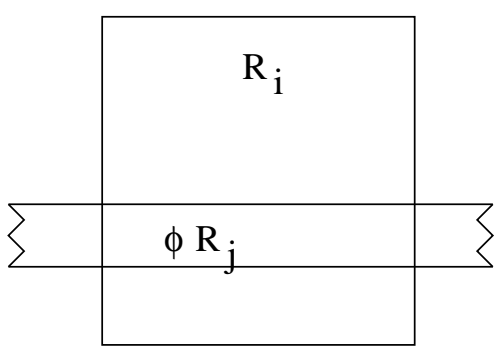

Possible

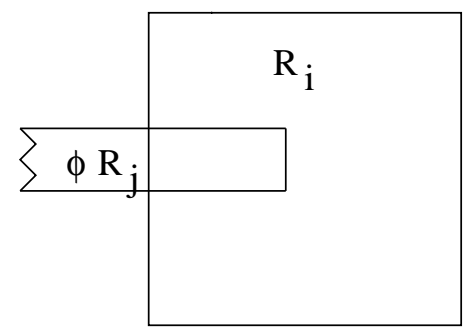

Impossible

FiguRE 12. Intersections

points which are $(1,0)$ and $(1,1)$ respectively. Thus the image of $\bar{d}$ is a, and the image of $\mathrm{a}$ is $\mathrm{c}$. These facts about the expanding and contracting boundaries imply that refinements of the original partition under positive iterates of $\phi$ do not have any new boundary segments in the $\mathrm{v}_{\mu}$-direction that are not already contained in $\mathrm{a} \overline{\mathrm{d}}$, while under negative iterates of $\phi$ there are no new ones in the $\mathrm{v}_{\lambda}$-direction not already in ob. From this we obtain that for $n \in \mathbb{N}$ a set $\phi^{n} R_{i} \cap R_{j}$, if non-empty, is a union of rectangles, each stretching in the expanding direction all the way across $R_{j}$. Similarly, a non-empty $R_{i} \cap \phi^{-n} R_{j}$ is a union of rectangles, each stretching in the contracting direction all the way across $R_{i}$. When $n=1$, it can be seen that each of these unions consists of a single rectangle (see Figure 12). This implies that if $\bigcap_{k=-n}^{0} \phi^{-k} R_{s_{k}} \neq \emptyset$, then this intersection is a single rectangle stretching all the way across $R_{s_{0}}$ in the expanding direction. Similarly, if $\bigcap_{k=0}^{n} \phi^{-k} R_{s_{k}} \neq \emptyset$, then this set is a single rectangle stretching all the way across $R_{s_{0}}$ in the contracting direction.

Combining these two results we have that a non-empty closed set of the form $\overline{\bigcap_{k=-n}^{n} \phi^{-k} R_{s_{k}}}$ is a closed rectangle. The diameter of these sets is uniformly bounded by constant $\times|\mu|^{n}$. Thus as $n \rightarrow \infty$, a sequence of such sets decreases to a point in $X$. Consequently, such a point can be represented by a sequence $s=\left(s_{n}\right)_{n \in \mathbb{Z}}$. If fact, all points of the torus can be so represented.

If $\bigcap_{k=-n}^{n} \phi^{-k} R_{s_{k}} \neq \emptyset$, then it is clear that $R_{s_{i}} \cap \phi^{-1} R_{s_{i+1}} \neq \emptyset$ for $-n \leq i \leq n-1$. The converse which is the Markov property is really the main one we are extracting from the geometry of this example. As we have seen $R_{s_{i}} \cap \phi^{-1} R_{s_{i+1}} \neq \emptyset$ if and only if edge $s_{i+1}$ follows edge $s_{i}$ according to the the graph of Figure 11. This means that the sequences $s=\left(s_{n}\right)_{n \in \mathbb{Z}}$ are elements is a topological Markov shift.

Once again sets $R_{i}$ have an obvious product structure. For $\mathrm{p} \in R_{i}$ we call the segment $h_{i}(\mathrm{p})$ specified by intersection of $R_{i}$ and the line through $p$ in the expanding direction the horizontal through p. Similarly, we refer to $v_{i}(\mathrm{p})$ given by the intersection of $R_{i}$ and the line through $p$ in the contraction direction as the vertical. Each rectangle is homeomorphic to the Cartesian product of any one of its horizontals with any one of its verticals. Just as for topological Markov shifts, the toral automorphism respects this structure: namely, for $\mathrm{p} \in R_{i} \cap \phi^{-1} R_{j}$ the 


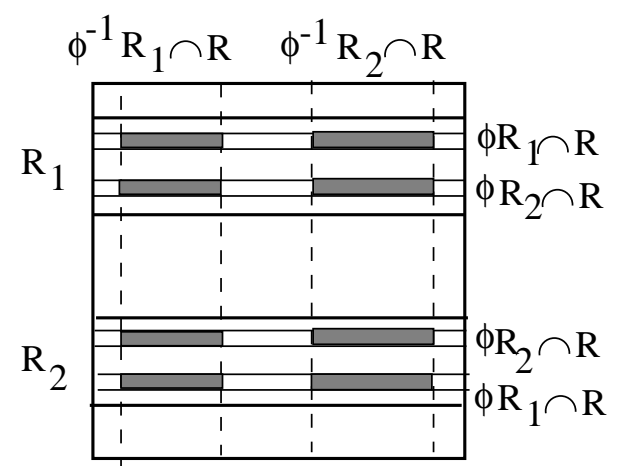

FIGURE 13. Sets containing the invariant set of the horseshoe map

following holds:

$$
\begin{aligned}
& \phi v_{i}(\mathrm{p}) \subset v_{j}(\phi \mathrm{p}), \\
& \phi h_{i}(\mathrm{p}) \supset h_{j}(\phi \mathrm{p}) .
\end{aligned}
$$

We shall incorporate what we have just described in a comprehensive theory.

3.4 Symbolism for the horseshoe map. The map $\phi$ indicated in Figure 4 has an invariant set $X=\bigcap_{n=-\infty}^{\infty} \phi^{n} R$.

As shown in Figure 13, let $R_{1}, R_{2}$ be the disconnected components of $\phi R \cap R$.

It can be proved that $X$ is homeomorphic to $\Sigma_{2}$ from which follows that $(X, \phi)$ and $\left(\Sigma_{2}, \sigma\right)$ are topologically conjugate.

For versions of horseshoe-like maps arising from cases where there are many homoclinic points linking various fixed points, the invariant sets can be specified by shifts of finite type. For the dynamical systems where the map is restricted to an invariant subset of its domain we have the exceptional situation where there is actually a one-to-one correspondence between their orbits and the symbolic sequences of a shift of finite type. Here the price paid is that not all of the domain of the map is represented by symbolic sequences but rather only an invariant subset.

\section{More on ABStract DyNAMiCAL Systems}

Definition 4.1. A dynamical system $(X, \phi)$ is said to be irreducible if for every pair of open sets $U, V$ there exists $n \geq 0$ such that $\phi^{n} U \cap V \neq \emptyset$.

Another concept we need is the following.

Definition 4.2. A point $\mathrm{p}$ is said to be bilaterally transitive if the forward orbit $\left\{\phi^{n} \mathrm{p} \mid n \geq 0\right\}$ and the backward orbit $\left\{\phi^{n} \mathrm{p} \mid n<0\right\}$ are both dense in $X$.

Remark. A symbolic sequence in a topological Markov shift is bilaterally transitive if every admissible block appears in both directions and infinitely often.

We use the notation $B L T(A)$ to denote the subset of bilaterally transitive points in $A \subseteq X$.

In an irreducible system the bilaterally transitive points turn out to be everywhere dense. To prove this, we recall the following theorem of point set topology. The theorem is more general, but can be slightly simplified in the case where the space $X$ is a compact metric space. 
Baire Category Theorem 4.3. Let $\left\{U_{n}\right\}$ be a countable collection of open dense subsets of $X$. Then $\bigcap U_{n}$ is non-empty. In fact $\cap U_{n}$ is dense in $X$. Equivalently, a compact metric space in not the union of a countable collection of nowhere dense sets.

Proof. Choose inductively balls $B_{n}$ such that $B_{n} \subset \overline{B_{n}} \subset U_{n}$, and $\overline{B_{n}} \subset B_{n-1}$. The first property is easily achieved in a metric space; the second because $U_{n}$ is dense, which implies that $B_{n-1} \cap U_{n}$ is a non-empty open set. The sequence $\left(\overline{B_{n}}\right)_{n \in \mathbb{N}}$ has the finite intersection property: so by compactness $\bigcap \overline{B_{n}}$ is non-empty. But $\bigcap \overline{B_{n}} \subset \bigcap B_{n} \subset \bigcap U_{n}$. Thus the intersection $\bigcap U_{n}$ is not empty. It is also dense, which is a consequence of replacing $U_{n}$ in the above argument by $U_{n} \cap B$ and $X$ by $\bar{B}$ where $B$ is any ball.

Proposition 4.4. If $(X, \phi)$ is irreducible, then the set of bilaterally transitive points is dense in $X$.

Proof. Let $\left\{U_{n}\right\}$ be a countable basis for $X$. Since $X$ is irreducible, $\bigcup_{k<0} \phi^{-k} U_{n}$, as well as $\bigcup_{k \geq 0} \phi^{-k} U_{n}$, is open dense in $X$ for each integer $n$. The set $B L T(X)$ of bilaterally transitive points can be expressed as

$$
B L T(X)=\bigcap_{n}\left(\bigcup_{k<0} \phi^{-k} U_{n} \cap \bigcup_{k \geq 0} \phi^{-k} U_{n}\right) .
$$

We apply the Baire Category Theorem to get $B L T(X) \neq \emptyset$. But $B L T(X)$ contains the whole orbit of any of its points, and the orbit of any of its points is dense in $X$.

The three examples we have discussed exhibit a certain property which is easily verified for them. It concerns the divergence of orbits and is defined as follows.

Definition 4.5. A homeomorphism $\phi$ is said to be expansive if there exists a real number $c>0$ such that if $d\left(\phi^{n} \mathrm{p}, \phi^{n} \mathrm{q}\right)<c$ for all $n \in \mathbb{Z}$, then $\mathrm{p}=\mathrm{q}$.

In the theory of Markov partitions this property plays a key role. Representation of dynamical systems by shifts of finite type is possible for certain non-expansive systems, but really seems natural only for expansive ones.

Next we formalize a property of mappings between dynamical systems previously alluded to in connection with binary expansions.

Definition 4.6. For two general dynamical systems $(X, \phi)$ and $(Y, \psi)$ we call the second a factor of the first and the first an extension of the second if there exists a map $\pi$ of $X$ into $Y$, which we call a factor map, such that

(i) $\psi \pi=\pi \phi$ (see Figure 14),

(ii) $\pi$ is continuous,

(iii) $\pi$ is onto.

Furthermore, we say $\pi$ is a finite factor map or that it is bounded-to-one if

(iv) there is a bound on the number of pre-images;

and essentially ${ }^{3}$ one-to-one if

(v) every doubly transitive point has a unique pre-image.

${ }^{3}$ This term is used because in irreducible systems the non-doubly transitive points are negligible in both the sense of category and measure. 
ROY L. ADLER

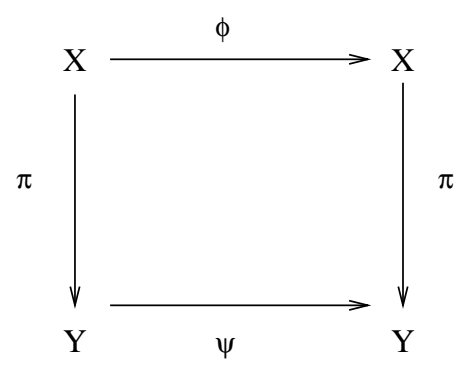

Figure 14. Commutative diagram illustrating a factor map

We remark that a topological conjugacy ${ }^{4}$ is a finite factor map where the bound on the number of pre-images is one in condition 1.2(iv). As we shall see, the seemingly slight weakening of the chains of topological conjugacy, which is what the definition of an essentially one-to-one finite factor map is meant to do, allows the necessary freedom to get symbolic representations for smooth dynamical systems.

Proposition 4.7. Let $\pi$ be a factor map of $(X, \phi)$ and $(Y, \psi)$ : i.e. $\pi$ satisfies properties (i), (ii), and (iii) of Definition 4.6. If $(X, \phi)$ is irreducible, then so is $(Y, \psi) ;$ and $Y=\overline{B L T(Y)}$.

Proof. Let $U, V$ be non-empty open subsets of $Y$. By properties (ii) and (iii) of factor maps, $\pi^{-1} U, \pi^{-1} V$ are also non-empty and open. Since $(X, \phi)$ is irreducible, there exists an integer $n>0$ such that $\phi^{n}\left(\pi^{-1} U\right) \cap \pi^{-1} V \neq \emptyset$. By 4.6(i),

$$
\emptyset \neq \pi\left[\phi^{n}\left(\pi^{-1} U\right) \cap \pi^{-1} V\right]=\pi\left[\pi^{-1}\left(\psi^{n} U \cap V\right)\right]=\psi^{n} U \cap V .
$$

Thus $(Y, \psi)$ is irreducible, and from Proposition 4.4 it follows that $Y=\overline{B L T(Y)}$.

Proposition 4.8. Let $(X, \phi)$ be irreducible and $\pi$ an essentially one-to-one factor map of $(X, \phi)$ onto $(Y, \psi)$ : i.e. $\pi$ satisfies (i),(ii),(iii), and (v) of Definition 4.6. Then $\pi$ maps BLT $(U)$ homeomorphically onto $B L T(\pi(U))$ for any open subset $U$ of $X$.

Proof. From the properties of $\pi$, if the forward orbit of $x$ hits every non-empty open subset of $X$, then the forward orbit of $\pi(x)$ hits every non-empty open subset of $Y$. Thus $\pi B L T(U) \subset B L T(\pi(U))$.

We have that $\pi$ is a continuous one-to-one map of $B L T(U)$ into $B L T(\pi(U))$. We prove next that its inverse is continuous also. The proof is a standard compactness argument which goes as follows. Suppose $y_{n} \rightarrow y$, where $y_{n}, y \in B L T(\pi(U))$. We shall prove that $\pi^{-1} y_{n} \rightarrow \pi^{-1} y$. By compactness the sequence $\left(\pi^{-1} y_{n}\right)_{n \in \mathbb{N}}$ has limit points in $X$. Let $x$ be any one of these limit points. By continuity $\pi x=y$. But the pre-image of $y$ is unique: so the sequence $\left(\pi^{-1} y_{n}\right)_{n \in \mathbb{N}}$, having only one limit point, has a limit which is $\pi^{-1} y$.

Now let $x \in \pi^{-1} B L T(\pi(U)) \subset U$, and let $V$ be any non-empty open subset of $X$. Choose $v \in B L T(V)$. Then by what we have already shown, $\pi(v) \in B L T(\pi(V))$.

${ }^{4}$ The term derives from the group theory notion of conjugate elements and its usage is standard in the subject. In the sense we are using it, better terms would have been homomorphism for factor map and isomorphism for topological conjugacy. These are the terms which denote the property of preserving structure. 
Then there exists a sequence of positive integers $k_{n}$ such that $\psi^{k_{n}} \pi(x) \rightarrow \pi(v)$. Thus

$$
\phi^{k_{n}}(x)=\pi^{-1} \psi^{k_{n}} \pi(x) \rightarrow \pi^{-1} \pi(v)=v .
$$

Thus $x \in B L T(U)$. We have therefore established $\pi^{-1} B L T(\pi(U)) \subset B L T(U):$ in other words, $B L T(\pi(U)) \subset \pi B L T(U)$.

Proposition 4.9. Under the hypothesis of Proposition 4.8, if $U$ is an open subset of $X$, then $B L T(\pi U)=B L T[\pi U]^{\circ}$ and $\pi \bar{U}=\overline{[\pi U]^{\circ}}$.

Proof. Let $y \in B L T(\pi U)$. Then the unique pre-image of $y$ lies in $B L T(U)$, and $\pi y$ is not therefore in the closed set $\pi(X-U)$. Hence, $\pi y \in Y-\pi(X-U) \subset[\pi U]^{\circ}$. Therefore, $B L T(\pi U)=B L T[\pi U]^{\circ}$.

From the continuity properties of $\pi$, Proposition 4.8, and what was just proven, we get the following string of equalities: $\pi(\bar{U})=\pi(\overline{B L T(U)})=\overline{B L T(\pi U)}=$ $\overline{B L T[\pi U]^{\circ}}=\overline{[\pi U]^{\circ}}$.

\section{Exercises.}

4.1 We call a directed graph $G$ irreducible if given any pair of nodes $i, j$ there is a directed path from $i$ to $j$. Show that if $G$ is irreducible as a graph, then the dynamical system $\left(\Sigma_{G}, \sigma\right)$ is irreducible. Conversely, show that if the dynamical system $\left(\Sigma_{G}, \sigma\right)$ is irreducible, then there is an irreducible subgraph $G^{\prime}$ such that $\Sigma_{G}=\Sigma_{G^{\prime}}$.

4.2 Given any topological Markov shift system $\left(\Sigma_{G}\right)$, there exist irreducible subgraphs $G_{1}, \ldots, G_{M}$ such that

$$
\Sigma_{G}=\Sigma_{G_{1}} \cup \cdots \cup \Sigma_{G_{M}}(\text { disjoint }) .
$$

\section{TOPOLOGICAL PARTITIONS}

Definition 5.1. We call a finite family of sets $\mathcal{R}=\left\{R_{0}, R_{1}, \ldots, R_{N-1}\right\}$ a topological partition for a compact metric space $X$ if:

(1) each $R_{i}$ is open ${ }^{5}$;

(2) $R_{i} \cap R_{i}=\emptyset, i \neq j$;

(3) $X=\overline{R_{0}} \cup \overline{R_{1}} \cup \cdots \cup \overline{R_{N-1}}$.

Remark. For open sets $U, V, \bar{U} \cap V \neq \emptyset \Rightarrow U \cap V \neq \emptyset$. So for members of a topological partition we get the following string of implications: $R_{i} \cap R_{j}=\emptyset \Rightarrow$ $\overline{R_{i}} \cap R_{j}=\emptyset \Rightarrow{\overline{R_{i}}}^{o} \cap R_{j}=\emptyset \Rightarrow{\overline{R_{i}}}^{o} \cap \overline{R_{j}}=\emptyset \Rightarrow{\overline{R_{i}}}^{o} \cap{\overline{R_{j}}}^{\circ}=\emptyset$. Thus ${\overline{R_{i}}}^{\circ} \cap{\overline{R_{j}}}^{\circ}=\emptyset$ for $i \neq j$.

Definition 5.2. Given two topological partitions $\mathcal{R}=\left\{R_{0}, R_{1}, \ldots, R_{N-1}\right\}$ and $\mathcal{S}=\left\{S_{0}, S_{1}, \ldots, S_{M-1}\right\}$, we define their common topological refinement $\mathcal{R} \vee \mathcal{S}$ as

$$
\mathcal{R} \vee \mathcal{S}=\left\{R_{i} \cap S_{j}: R_{i} \in \mathcal{R}, S_{j} \in \mathcal{S}\right\} .
$$

Proposition 5.3. The common topological refinement of two topological partitions is a topological partition.

\footnotetext{
${ }^{5}$ Previous authors have taken these sets to be closed sets with the property that each is the closure of its interior. The present variation is slightly more general, just enough to make some notation and certain arguments simpler. In fact, an important example is presented in Section 9 of a partition whose elements are not the interiors of their closures.
} 
ROY L. ADLER

Proof. Let $\mathcal{R}$ and $\mathcal{S}$ be the two partitions in question. First of all, it is clear that the elements of $\mathcal{R} \vee \mathcal{S}$ are disjoint. We show that the closure of elements of $\mathcal{R} \vee \mathcal{S}$ cover $X$. Let $\mathrm{p} \in X$. We have that $\mathrm{p} \in \overline{R_{i}}$ for some $i$. Thus there exists a sequence of points $\mathrm{p}_{n} \in R_{i}$ such that $d\left(\mathrm{p}_{n}, \mathrm{p}\right)<1 / n$. Since $\mathcal{S}$ is a topological partition, for each $n$ there exists $S_{j_{n}} \in \mathcal{S}$ such that $\mathrm{p}_{n} \in \overline{S_{j_{n}}}$. Since $\mathcal{S}$ is finite, there exists an index $j$ such that $j_{n}=j$ for an infinite number of $n$ so that we can assume that the $\mathrm{p}_{n}$ were chosen in the first place such that each $j_{n}=j$. Since $\mathrm{p}_{n} \in R_{i} \cap \overline{S_{j}}$ we can choose a sequence of points $\mathrm{q}_{m, n} \in R_{i} \cap S_{j}$ such that $d\left(\mathrm{q}_{m, n}, \mathrm{p}_{n}\right)<1 / m$. Thus $d\left(\mathrm{q}_{n, n}, \mathrm{p}\right)<2 / n$; whence $\mathrm{q}_{n, n} \rightarrow \mathrm{p}$ as $n \rightarrow \infty$. Therefore $\mathrm{p} \in \overline{R_{i} \cap S_{j}}$.

Proposition 5.4. For dynamical system $(X, \phi)$ with topological partition $\mathcal{R}$ of $X$, the set $\phi^{n} \mathcal{R}$ defined by $\phi^{n} \mathcal{R}=\left\{\phi^{n} R_{1}, \ldots, \phi^{n} R_{N-1}\right\}$ is again a topological partition.

Proof. This is an immediate consequence of the following: (1) the image of a union is the union of images for any map, (2) a homeomorphism commutes with the operation of taking closures, (3) the image of an intersection is the intersection of images for a one-one map.

From Proposition 5.3 and 5.4 we have that for $m \leq n, \bigvee_{m}^{n} \phi^{k} \mathcal{R}=\phi^{m} \mathcal{R} \vee$ $\phi^{m-1} \mathcal{R} \vee \cdots \vee \phi^{n} \mathcal{R}$ is again a topological partition. We shall use the notation

$$
\mathcal{R}^{(n)} \equiv \bigvee_{k=0}^{n-1} \phi^{-k} \mathcal{R}
$$

Thus $\mathcal{R}^{(2)}=\mathcal{R} \vee \phi^{-1} \mathcal{R}=\left\{R_{i} \cap \phi^{-1} R_{j}: R_{i}, R_{j} \in \mathcal{R}\right\}$. Observe that $\left(\mathcal{R}^{(2)}\right)^{(2)}=\mathcal{R}^{(3)}$, or more generally $\left(\mathcal{R}^{(n)}\right)^{(m)}=\mathcal{R}^{(n+m-1)}$.

The collection $\bigcup \phi^{n} \mathcal{R}: n \in \mathbb{Z}$ is a collection of open dense sets to which we can apply the Baire theorem, but due to its special nature we can achieve a slightly stronger result with the same sort of proof.

Proposition 5.5. Let $\mathcal{R}$ be a topological partition for dynamical system $(X, \phi)$. For every $\mathrm{p} \in X$ there exists a sequence $\left(R_{s_{k}}\right)_{k \in \mathbb{Z}}$ of sets in $\mathcal{R}$ such that $\mathrm{p} \in$ $\bigcap_{n=0}^{\infty} \overline{\bigcap_{-n}^{n} \phi^{-k} R_{s_{k}}}$.

Proof. Since $\bigvee_{m}^{n} \phi^{k} \mathcal{R}, m \leq n$, is a topological partition, there is a set in it whose closure contains p, say $\overline{\bigcap_{m}^{n} \phi^{-k} R_{s_{k}}}$. We next show that in the refinement $\bigvee_{m-1}^{n+1} \phi^{k} \mathcal{R}$ the elements of the form $\bigcap_{m-1}^{n+1} \phi^{-k} R_{t_{k}}$ where $t_{k}=s_{k}$ for $m \leq k \leq n$ comprise a subfamily which is a topological partition of $\overline{\bigcap_{m}^{n} \phi^{-k} R_{s_{k}}}$. Because $\bigvee_{m-1}^{n+1} \phi^{k} \mathcal{R}$ satisfies 5.1(1) and (2), so does any subfamily. Condition 5.1(3) is a consequence of

$$
\bigcup_{\substack{0 \leq t_{m-1} \leq N-1 \\ 0 \leq t_{n+1} \leq N-1 \\ t_{k}=s_{k}, m \leq k \leq n}} \bigcap_{m-1}^{n+1} \phi^{-k} R_{t_{k}}=\bigcap_{m}^{n} \phi^{-k} R_{s_{k}}
$$

and the fact that the closure of a union is the union of closures. Thus we can choose by induction the sets $R_{s_{k}}$ as follows. Once having specified sets $R_{s_{-n}}, \ldots, R_{s_{n}}$ such that $\mathrm{p} \in \overline{\bigcap_{-n}^{n} \phi^{-k} R_{s_{k}}}$, we can find sets $R_{s_{-n-1}}$ and $R_{s_{n+1}}$ such that $\mathrm{p} \in$ $\overline{\bigcap_{-n-1}^{n+1} \phi^{-k} R_{s_{k}}}$. Hence there exists a sequence $\left(R_{s_{k}}\right)_{k \in \mathbb{Z}}$ of sets in $\mathcal{R}$ such that $\mathrm{p} \in \bigcap_{n=0}^{\infty} \overline{\bigcap_{-n}^{n} \phi^{-k} R_{s_{k}}}$. 
Remark. With a slight modification of this proof somewhat more can be established: namely, a finite sequence of sets $R_{s_{m}}, R_{s_{m+1}}, \ldots, R_{s_{n}}$ can be extended to a bi-infinite sequence $\left(R_{s_{n}}\right)_{n \in \mathbb{Z}}$ such that if $\mathrm{p} \in \overline{\bigcap_{m}^{n} \phi^{-k} R_{s_{k}}}, m \leq n$, then $\mathrm{p} \in \bigcap_{n=0}^{\infty} \overline{\bigcap_{-n}^{n} \phi^{-k} R_{s_{k}}}$. We can even go further and make the same claim about extending a one-sided infinite sequence $R_{s_{m}}, R_{s_{m+1}}, \ldots$ to a bi-infinite one.

Definition 5.6. We define the diameter $d(\mathcal{R})$ of a partition $\mathcal{R}$ by

$$
d(\mathcal{R})=\max _{R_{i} \in \mathcal{R}} d\left(R_{i}\right)
$$

where $d\left(R_{i}\right) \equiv \sup _{x, y \in R_{i}} d(x, y)$.

Definition 5.7. We call a topological partition a generator for a dynamical system $(X, \phi)$ if $\lim _{n \rightarrow \infty} d\left(\bigvee_{-n}^{n} \phi^{k} \mathcal{R}\right)=0$.

If $\mathcal{R}$ is a generator, then clearly $\lim _{n \rightarrow \infty} d\left(\bigcap_{-n}^{n} \phi^{-k} R_{s_{k}}\right)=0$ for any sequence of symbols $\left(s_{i}\right)_{i \in \mathbb{Z}} \in\{0, \ldots, N-1\}^{\mathbb{Z}}$. The converse is also true (see Exercise 5.1). In addition $d\left(\bigcap_{n=0}^{\infty} \overline{\bigcap_{-n}^{n} \phi^{-k} R_{s_{k}}}\right)=0$. Hence in Proposition 5.5, if $\mathcal{R}$ is a generator and $\mathrm{p} \in \bigcap_{n=0}^{\infty} \overline{\bigcap_{-n}^{n} \phi^{-k} R_{s_{k}}}$, then $\mathrm{p}=\bigcap_{n=0}^{\infty} \overline{\bigcap_{-n}^{n} \phi^{-k} R_{s_{k}}}$.

The following proposition gives sufficient conditions on a topological partition in terms of its diameter for it to be a generator.

Proposition 5.8. Let $(X, \phi)$ be expansive and $\mathcal{R}$ be a topological partition such that $d(\mathcal{R})<c$ where $c$ is the expansive constant. Then $\mathcal{R}$ is a generator.

Proof. The set $\bigcap_{-\infty}^{\infty} \phi^{-k} \overline{R_{s_{k}}}$ contains at most one point and thus has zero diameter: for if there exists $\mathrm{p}, \mathrm{q} \in \bigcap_{-\infty}^{\infty} \phi^{-k} \overline{R_{s_{k}}}$, then $d\left(\phi^{n} \mathrm{p}, \phi^{n} \mathrm{q}\right)<c$ for $n \in \mathbb{Z}$ implying p $=$ q. Since $\bigcap_{-n}^{n} \phi^{-k} R_{s_{k}} \subset \bigcap_{-n}^{n} \phi^{-k} \overline{R_{s_{k}}}, d\left(\lim _{n \rightarrow \infty} \bigcap_{-n}^{n} \phi^{-k} R_{s_{k}}\right)=$ $d\left(\bigcap_{-\infty}^{\infty} \phi^{-k} \overline{R_{s_{k}}}\right)=0$. From Exercise 5.1 we get that $\mathcal{R}$ is a generator.

Remarks. Generally we merely have the inclusion relation

$$
\bigcap_{n=0}^{\infty} \overline{\bigcap_{-n}^{n} \phi^{-k} R_{s_{k}}} \subset \bigcap_{-\infty}^{\infty} \phi^{-k} \overline{R_{s_{k}}}
$$

but not equality. However, when the sets of the partition are small enough-namely, when the hypothesis of Proposition 5.8 is satisfied- we do have equality: that is, if $\bigcap_{n=0}^{\infty} \overline{\bigcap_{-n}^{n} \phi^{-k} R_{s_{k}}} \neq \emptyset$, then $\bigcap_{n=0}^{\infty} \overline{\bigcap_{-n}^{n} \phi^{-k} R_{s_{k}}}=\bigcap_{-\infty}^{\infty} \phi^{-k} \overline{R_{s_{k}}}$.

From the inclusion relation (5.9) we see that if $\pi(s)=\mathrm{p}$, then $\mathrm{p} \in \overline{R_{s_{0}}}$. Thus if $x$ belongs only to $\overline{R_{i}}$, then $s_{0}=i$. In particular, by the remark following Definition 5.1 , if $\mathrm{p} \in R_{i}$ or $\mathrm{p} \in{\overline{R_{i}}}^{\circ}$, then $s_{0}=i$. In addition, if there exist sequences $s, t$ such that $\pi(s)=\pi(t)=\mathrm{p}$ and $s_{0}=i \neq j=t_{0}$, then $\mathrm{p} \in \overline{R_{i}} \cap \overline{R_{j}}, i \neq j$, and conversely. In which case $\mathrm{p} \in\left(\overline{R_{i}}-{\overline{R_{i}}}^{\circ}\right) \cap\left(\overline{R_{j}}-{\overline{R_{j}}}^{o}\right)=\partial R_{i} \cap \partial R_{j}$ : i.e. p belongs to the boundary of partition elements.

Let $\mathcal{R}=\left\{R_{1}, \ldots, R_{N}\right\}$ be a generator for a dynamical system $(X, \phi)$. Let $\Sigma$ be the subset of the full $N$-shift defined by

$$
\Sigma \equiv\left\{s=\left(\ldots, s_{n}, \ldots\right): \bigcap_{n=0}^{\infty} \overline{\bigcap_{-n}^{n} \phi^{-k} R_{s_{k}}} \neq \emptyset\right\} .
$$




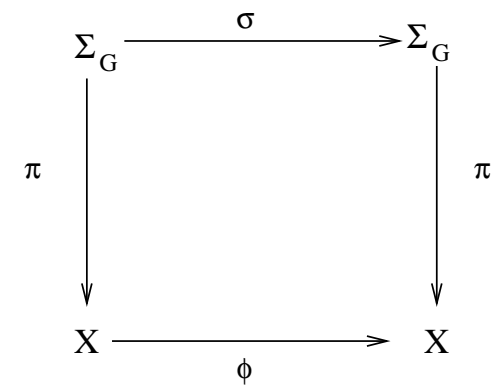

FiguRE 15. Commutative diagram for symbolic representation

Because the topological partition $\mathcal{R}$ is a generator, the non-empty infinite intersection $\bigcap_{n=0}^{\infty} \overline{\bigcap_{k=-n}^{n} \phi^{-k} R_{s_{k}}}$ consists of a single point. Therefore, we can define a $\operatorname{map} \pi: \Sigma \longrightarrow X$ by

$$
\pi(s)=\bigcap_{n=0}^{\infty} \frac{\phi^{n} R_{s_{-n}} \cap \phi^{n-1} R_{s_{-n+1}} \cap \cdots \cap \phi^{-n} R_{s_{n}}}{} .
$$

Figure 15 helps us to keep in mind what is being mapped to what.

Proposition 5.12. Let the dynamical system $(X, \phi)$ have a topological partition $\mathcal{R}$ which is a generator. Then $\Sigma$ as defined by (5.10) is a closed shift-invariant subset of $\Sigma_{N}$ and the map $\pi$ given by (5.11) is a factor map of the dynamical system $(\Sigma, \sigma)$ onto $(X, \phi)--$ i.e., $\pi$ satisfies the following items of Definition 4.6:

(i) $\sigma \pi=\pi \phi$,

(ii) $\pi$ is continuous,

(iii) $\pi$ is onto.

Proof. To prove $\Sigma$ is closed we must show that if $s=\left(\ldots, s_{k}, \ldots\right) \in \bar{\Sigma}$, then

$$
\bigcap_{n=0}^{\infty} \overline{\bigcap_{-n}^{n} \phi^{-k} R_{s_{k}}} \neq \emptyset
$$

which then implies that $s \in \Sigma$. For each $n>1$ there is a sequence $t=\left(\ldots, t_{k}, \ldots\right) \in$ $\Sigma$ such that $d(t, s)<1 / n$. This means that $t_{k}=s_{k},-n \leq k \leq n$ so that

$$
\overline{\bigcap_{-n}^{n} \phi^{-k} R_{t_{k}}}=\overline{\bigcap_{-n}^{n} \phi^{-k} R_{s_{k}}}
$$

Because $t \in \Sigma$, this set is non-empty. Since this is so for arbitrary $n$ and these sets form a decreasing sequence of non-empty closed sets, applying compactness we get 5.13 .

To prove $\Sigma$ is $\sigma$-invariant we must show that if $s \in \Sigma$, then $\sigma s \in \Sigma$ : in other words, for $n \geq 0$, if $\bigcap_{-n}^{n} \phi^{-k} R_{s_{k}} \neq \emptyset$, then $\bigcap_{-n}^{n} \phi^{-k} R_{s_{k+1}} \neq \emptyset$. This follows from using the distributive property of $\phi^{-1}$ with respect to intersections and reindexing: i.e.

$$
\phi^{-1}\left(\bigcap_{-n}^{n} \phi^{-k} R_{s_{k+1}}\right)=\bigcap_{-n+1}^{n+1} \phi^{-k} R_{s_{k}} \supset \bigcap_{-n+1}^{n-1} \phi^{-k} R_{s_{k}} .
$$

We now turn our attention to the properties of $\pi$. 
(i) $\pi$ satisfies $\pi \sigma=\phi \pi$. This follows from reindexing after applying the property that a homeomorphism commutes with the closure operation and preserves intersections: to wit,

$$
\begin{aligned}
\phi \pi(s) & =\phi \bigcap_{n=0-n}^{\infty} \overline{\bigcap^{-k} R_{s_{k}}} \\
& =\bigcap_{n=0}^{\infty} \overline{\bigcap_{n}^{n} \phi^{-k+1} R_{s_{k}}} \\
& =\bigcap_{n=0}^{\infty} \overline{\bigcap_{-n-1}^{n-1} \phi^{-k} R_{s_{k+1}}} \\
& =\bigcap_{n=0}^{\infty} \overline{\bigcap_{-n+1}^{n-1} \phi^{-k} R_{s_{k+1}}}=\pi(\sigma s) .
\end{aligned}
$$

(ii) $\pi$ is continuous. From the generating property of $\mathcal{R}$, given $\epsilon>0$ there is a positive integer $n$ such that $d\left(\overline{\bigcap_{-n}^{n} \phi^{-k} R_{s_{k}}}\right)<\epsilon$. Thus, for $s, t \in \Sigma_{G}$, there is a $\delta>0$, namely $\delta=1 /(n+1)$, such that if $d(s, t)<\delta$, then $\pi(s), \pi(t) \in \overline{\bigcap_{-n}^{n} \phi^{-k} R_{s_{k}}}$.

(iii) $\pi$ is onto. This follows immediately from Proposition 5.5.

\section{Exercises.}

5.1 Let $\mathcal{R}$ be a topological partition for a dynamical system $(X, \phi)$. Prove that if $\lim _{n \rightarrow \infty} d\left(\bigcap_{-n}^{n} \phi^{-k} R_{s_{k}}\right)=0$ for any sequence of symbols $\left(s_{i}\right)_{i \in \mathbb{Z}} \in\{0, \ldots$, $N-1\}^{\mathbb{Z}}$, then $\lim _{n \rightarrow \infty} d\left(\bigvee_{-n}^{n} \phi^{k} \mathcal{R}\right)=0$.

\section{MARKOV PARTITIONS AND SYMBOLIC EXTENSIONS}

Definition 6.1. We say that a topological partition $\mathcal{R}$ for a dynamical system $(X, \phi)$ satisfies the $n$-fold intersection property for a positive integer $n \geq 3$ if

$$
R_{s_{k}} \cap \phi^{-1} R_{s_{k+1}} \neq \emptyset, 1 \leq k \leq n-1 \Rightarrow \bigcap_{k=1}^{n} \phi^{-k} R_{s_{k}} \neq \emptyset .
$$

Furthermore, we call a topological partition Markov if it satisfies the $n$-fold intersection property for all $n \geq 3$.

Remark. In Section 3.1 and before the term "Markov" topological generator was defined, we considered the partition $\mathcal{C}=\left\{C_{a}: a \in A\right\}$ consisting of the elementary cylinder sets $C_{a}=\left\{s \in \Sigma_{G}: s_{0}=a\right\}$ for a dynamical system $\left(\Sigma_{G}, \sigma\right)$ where $\Sigma_{G}$ is a shift of finite type base on an alphabet $A$. As one might have guessed this partition is the prototype of a topological Markov generator.

Proposition 6.2. If $\mathcal{R}$ is a Markov partition, then so is $\bigvee_{m}^{n} \phi^{k} \mathcal{R}$ for any $m \leq n$.

Proof. We leave the proof as an exercise.

If a topological partition $\mathcal{R}$ satisfies the $n$-fold intersection property, then it satisfies $k$-fold ones for all smaller $k$. To increase the order we shall utilize the following.

Bootstrap Lemma 6.3. If $\mathcal{R}$ satisfies the 3-fold and $\mathcal{R}^{(2)}$ satisfies the $n$-fold intersection properties, $n \geq 3$, then $\mathcal{R}$ satisfies the $(n+1)$-fold intersection property. 
ROY L. ADLER

Proof. Suppose $R_{i_{k}} \cap \phi^{-1} R_{i_{k+1}} \neq \emptyset, 1 \leq k \leq n$. Because $\mathcal{R}$ satisfies the 3 -fold intersection property, we have

$$
R_{i_{k}} \cap \phi^{-1} R_{i_{k+1}} \cap \phi^{-2} R_{i_{k+2}} \neq \emptyset, 1 \leq k \leq n-1 .
$$

In other words,

$$
\left(R_{i_{k}} \cap \phi^{-1} R_{i_{k+1}}\right) \cap \phi^{-1}\left(R_{i_{k+1}} \cap \phi^{-1} R_{i_{k+2}}\right) \neq \emptyset, 1 \leq k \leq n-1 .
$$

Because $\mathcal{R}^{(2)}$ satisfies the $n$-fold intersection property, we obtain

$$
\bigcap_{1}^{n+1} \phi^{-k} R_{i_{k}}=\bigcap_{1}^{n} \phi^{-k}\left(R_{i_{k}} \cap \phi^{-1} R_{i_{k+1}}\right) \neq \emptyset .
$$

Suppose a dynamical system $(X, \phi)$ has a Markov generator $\mathcal{R}=\left\{R_{0}, \ldots, R_{N-1}\right\}$. We define an associated topological Markov shift given by the directed graph $G$ whose vertices are labelled by $\mathcal{A}=\{0,1, \ldots, N-1\}$ and in which the $i$-th vertex is connected to the $j$-th, $i \rightarrow j$, iff $R_{i} \cap \phi^{-1} R_{j} \neq \emptyset$. So by definition of the Markov shift associated with a transition matrix of a directed graph,

$$
\Sigma_{G}=\left\{s=\left(s_{n}\right)_{n \in \mathbb{Z}}: R_{s_{n-1}} \cap \phi^{-1} R_{s_{n}} \neq \emptyset, s_{n} \in \mathcal{A}, n \in \mathbb{Z}\right\} .
$$

This set coincides with the subsystem defined by 5.10 , which is easily seen as follows. On one hand, for $s \in \Sigma_{G}$, each of the closed sets

$$
\left\{\overline{\bigcap_{k=-n}^{n} \phi^{-k} R_{s_{k}}} \mid n=1,2 \ldots\right\}
$$

for any $n \geq 0$ is non-empty since the finite intersection under the closure sign is non-empty due to the Markov property. For increasing $n$ these closed intersections form a decreasing sequence of non-empty sets, and therefore by compactness $\bigcap_{n=0}^{\infty} \overline{\bigcap_{k=-n}^{n} \phi^{-k} R_{s_{k}}} \neq \emptyset$. On the other hand, if $\bigcap_{n=0}^{\infty} \overline{\bigcap_{k=-n}^{n} \phi^{-k} R_{s_{k}}} \neq \emptyset$, then each finite intersection under the closure sign is non-empty, which in turn implies that each pair of intersections $R_{s_{k}} \cap \phi^{-1} R_{s_{k}} \neq \emptyset$, for arbitrary $k \in \mathbb{Z}$.

\section{Main Theorem.}

Theorem 6.5. Suppose the dynamical system $(X, \phi)$ is expansive and has a Markov generator $\mathcal{R}=\left\{R_{0}, \ldots, R_{N-1}\right\}$. Then the map $\pi$, as defined by (5.11), is an essentially one-to-one finite factor map of the shift of finite type $\Sigma_{G}$, as defined by (6.1), onto $X$. Furthermore, if $(X, \phi)$ is irreducible, then so is $\left(\Sigma_{G}, \sigma\right)$.

Proof. We must establish (i) - (v) in Definition 4.6. That $\pi$ is a factor map-namely, it satisfies items (i), (ii), and (iii)-is the content of Theorem 5.12.

In order to establish (iv)-namely, a bound on the number of pre-images under $\pi$-we introduce the following concept.

Definition 6.6. A map $\pi$ from $\Sigma_{G}$ to $X$ is said to have a diamond if there are two sequences $s, t \in \Sigma_{G}$ for which $\pi(s)=\pi(t)$ and for which there exist indices $k<l<m$ such that $s_{k}=t_{k}, s_{l} \neq t_{l}, s_{m}=t_{m}$. See Figure 16 .

Lemma 6.7. If the number of pre-images of a point is more than $N^{2}$, then $\pi$ has a diamond. 


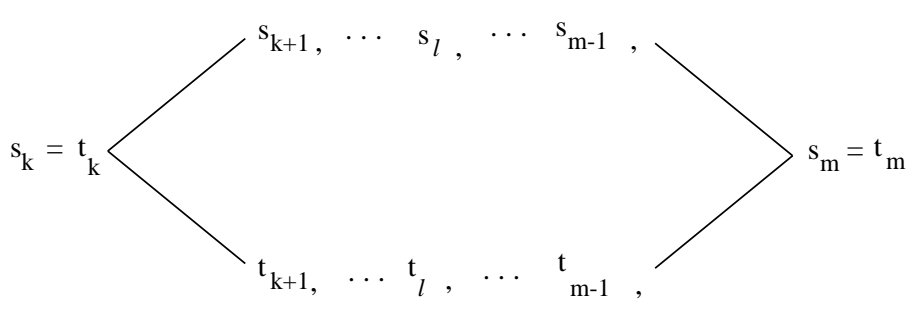

Figure 16. A diamond

Proof. We apply the familiar "pigeon hole" argument. Let $s^{(1)}, \ldots s^{\left(N^{2}+1\right)}$ be $N^{2}+1$ different sequences which map to the same point. Since the sequences are distinct, there are a pair of indices $k, m$ such that the allowable blocks $s_{[k, m]}^{(1)}, \ldots s_{[k, m]}^{\left(N^{2}+1\right)}$ are distinct. There are $N^{2}$ distinct choices of pairs of symbols $\left(s_{k}^{(i)}, s_{m}^{(i)}\right)$ : so by the "pigeon hole principle" there must be two allowable blocks $s_{[k, m]}^{(i)}, s_{[k, m]}^{(j)}$, such that $\left(s_{k}^{(i)}, s_{m}^{(i)}\right)=\left(s_{k}^{(j)}, s_{m}^{(j)}\right)$. But, since the blocks are different, there is an index $l$ such that $s_{l}^{(i)} \neq s_{l}^{(j)}$. Thus the two sequences $s^{(i)}, s^{(j)}$ map to the same point, agree at indices $k, m$, but differ at $l$ which is between $k, m$, which means there is a diamond.

Lemma 6.8. If there exists a bilaterally transitive point with two pre-images, then $\pi$ has a diamond.

Proof. Let a $B L T$ point p have two pre-images. As we have indicated in the remark following 5.8, there are two sets $R_{a}, R_{b} \in \mathcal{R}, a \neq b$, such that $\mathrm{p} \in \overline{R_{a}} \cap \overline{R_{b}}$. For each $n>0$, the family of sets

$$
\left\{\overline{\phi^{n} R_{s_{-n}} \cap \cdots \cap R_{s_{0}} \cap \ldots \phi^{-n} R_{s_{n}}}: s \in \Sigma_{G} \quad \text { where } \quad s_{0}=a\right\}
$$

covers $\overline{R_{a}}$, and the family

$$
\left\{\overline{\phi^{n} R_{t_{-n}} \cap \cdots \cap R_{t_{0}} \cap \ldots \phi^{-n} R_{t_{n}}}: t \in \Sigma_{G} \quad \text { where } \quad t_{0}=b\right\}
$$

covers $\overline{R_{b}}$. Thus, by compactness, there exists $s, t \in \Sigma_{G}$ with $s_{0}=a, t_{0}=b$ such that

$$
\mathrm{p} \in \bigcap_{n=0}^{\infty} \overline{\bigcap_{-n}^{n} \phi^{-k} R_{s_{k}}}
$$

and

$$
\mathrm{p} \in \bigcap_{n=0}^{\infty} \overline{\bigcap_{-n}^{n} \phi^{-k} R_{t_{k}}} .
$$

Since $\mathrm{p}$ is bilaterally transitive and $R_{0}$ is open, $\phi^{n} \mathrm{p} \in R_{0}$ for some positive $n$ and $\phi^{m} \mathrm{p} \in R_{0}$ for some negative $m$. Thus by the remark following (5.8), $s_{m}=t_{m}=$ $0, s_{0}=a \neq b=t_{0}, s_{n}=t_{n}=0$, which is a diamond for $\pi$.

Lemma 6.9. If $d(\mathcal{R})<c / 2$, then $\pi$ has no diamonds.

Proof. Since $\pi \sigma=\phi \pi$, we can assume without loss of generality that $k=1$ in the definition of a diamond. Assume that $\mathrm{p}=\pi(s)=\pi(t)$ where

$$
s=\left(\ldots, s_{-2}, a, b_{0}, b_{1}, \ldots, b_{m-1}, d, s_{m+1}, \ldots\right),
$$


ROY L. ADLER

$$
t=\left(\ldots, t_{-2}, a, c_{0}, c_{1}, \ldots, c_{m-1}, d, t_{m+1}, \ldots\right) .
$$

We must show that $b_{l}=c_{l}$ for $0 \leq l \leq m-1$. Because $\left[a, b_{0}, b_{1}, \ldots, b_{m-1}, d\right]$ is an allowable block in $\Sigma_{G}$,

$$
\phi R_{a} \cap R_{b_{0}} \cap \phi^{-1} R_{b_{1}} \cap \cdots \cap \phi^{-m+1} R_{m-1} \cap \phi^{-m} R_{d} \neq \emptyset .
$$

Choose a point $\mathrm{q}$ in this open set. Because $\pi$ is onto, there is a sequence

$$
u=\left(\ldots, u_{-2}, a, b_{0}, b_{1}, \ldots, b_{m-1}, d, u_{m+1}, \ldots\right) \in \Sigma_{G}
$$

such that $\pi(u)=\mathrm{q}$. Also since $\left[a, c_{0}, c_{1}, \ldots, c_{m-1}, d\right]$ is an allowable block and $\Sigma_{G}$ is a shift of finite type, there is a sequence $v \in \Sigma_{G}$ such that

$$
v=\left(\ldots, u_{-2}, a, c_{0}, c_{1}, \ldots, c_{m-1}, d, u_{m+1}, \ldots\right) .
$$

Thus

$$
\mathrm{r} \equiv \pi(v) \in \overline{\phi R_{a} \cap R_{c_{0}} \cap \phi^{-1} R_{c_{1}} \cap \cdots \cap \phi^{-m+1} R_{m-1} \cap \phi^{-m} R_{d}} .
$$

From $d\left(R_{i}\right)<c / 2$ and $\phi^{l}(x) \in \overline{R_{b_{l}}} \cap \overline{R_{c_{l}}}$ for $0 \leq l \leq m-1$, we conclude by the triangle inequality that $d\left(\phi^{l} \mathrm{q}, \phi^{l} \mathrm{r}\right)<c$. Furthermore, $d\left(\phi^{n} \mathrm{q}, \phi^{n} \mathrm{r}\right)<c / 2$ for $n<0$ and $n>m-1$. The expansive property then implies that $\mathrm{q}=\mathrm{r}$. Thus $R_{b_{l}} \cap \overline{R_{c_{l}}} \neq \emptyset$ which implies that $R_{b_{l}} \cap R_{c_{l}} \neq \emptyset$. However, elements of $\mathcal{R}$ are pairwise disjoint: so $b_{l}=c_{l}$.

(iv) There is a bound on the number of pre-images of $\pi$.

(v) A BLT point has a unique pre-image.

Because $\mathcal{R}$ is a generator, $n$ can be chosen so that $d\left(\bigvee_{-n}^{n} \phi^{k} \mathcal{R}\right)<c / 2$. By Proposition 6.2, $\bigvee_{-n}^{n} \phi^{k} \mathcal{R}$ is again a topological Markov partition. For this partition the associated shift of finite type of (6.4) is given by the higher edge graph $G^{(2 n+1)}$. Let $\pi^{(2 n+1)}$ be the map of $\Sigma_{G^{(2 n+1)}}$ onto $X$ according to (5.11). It has no diamonds: so by Lemma 6.7 a point has at most $N^{2(2 n+1)}$ pre-images, and by Lemma 6.8 a $B L T$ point has only one. The original $\pi$ satisfies $\pi=\pi^{(2 n+1)} \psi \sigma^{n}$ where $\psi$ is a conjugacy of $\Sigma_{G}$ onto $\Sigma_{G^{(2 n+1)}}$. Thus we have that under $\pi$ a point has at most $N^{2(2 n+1)}$ pre-images, and a $B L T$ point has a unique pre-image.

We defer the proof of irreducibility to Exercise 6.2.

Converse to the Main Theorem. Recall that we introduced in 3.1 the partition $\mathcal{C}=\left\{C_{i}: i=0, \ldots, N-1\right\}$ consisting of the elementary cylinder sets $C_{i}=\{s \in$ $\left.\Sigma_{G}: s_{0}=i\right\}$ for a dynamical system $\left(\Sigma_{G}, \sigma\right)$ where $\Sigma_{G}$ is a shift of finite type based on an alphabet $A=\{0,1, \ldots, N-1\}$. This partition is a topological Markov generator.

Theorem 6.10. Let $(X, \phi)$ be a dynamical system, $\left(\Sigma_{G}, \sigma\right)$ an irreducible shift of finite type based on $N$ symbols, and suppose there exists an essentially one-to-one factor map $\pi$ from $\Sigma_{G}$ to $X$. Then the partition $\mathcal{R}$ defined by $\mathcal{R}=\left\{R_{i}=\pi\left(C_{i}\right)^{o}\right.$ : $i=0, \ldots, N-1\}$ is a topological Markov generator.

Remark. Note we assume $\pi$ is a factor map which has a unique inverse for each bilaterally transitive point, but no bound is assumed on the number of pre-images of arbitrary points: i.e., $\pi$ satisfies (i), (ii), (iii), and (v) of Definition 4.6 but not (iv). However, in Corollary 6.12 we shall show that (iv) follows from the others under the hypothesis of expansivity. However, as Exercise 6.3 shows property (v) is essential: we cannot obtain it from expansivity and (i) through (iv). 
Proof. We must prove the following items:

1. Elements of $\mathcal{R}$ are disjoint.

2. The closure of elements of $\mathcal{R}$ cover $X$.

3. $\mathcal{R}$ is a generator.

4. $\mathcal{R}$ satisfies the Markov property.

(1) Elements of $\mathcal{R}$ are disjoint: i.e., $R_{i} \cap R_{j}=\emptyset, i \neq j$.

The idea of the proof is to use bilaterally transitive points to overcome a difficulty: namely, maps in general do not enjoy the property that the image of an intersection is equal to the intersection of images, but one-to-one maps do. Suppose $R_{i} \cap R_{j} \neq$ $\emptyset$ for $i \neq j$. Then, by Proposition $4.7 B L T\left(R_{i} \cap R_{j}\right) \neq \emptyset$. By Propositions 4.8 and $4.9, \pi^{-1}$ maps $B L T\left(R_{i}\right)$ and $B L T\left(R_{j}\right)$ homeomorphically onto $B L T\left(C_{i}\right)$ and $B L T\left(C_{j}\right)$ respectively. Therefore $\pi^{-1}$ maps $B L T\left(R_{i} \cap R_{j}\right)=B L T\left(R_{i}\right) \cap B L T\left(R_{j}\right)$ homeomorphically onto $B L T\left(C_{i} \cap C_{j}\right)=B L T\left(C_{i}\right) \cap B L T\left(C_{j}\right)$, which implies that $\emptyset \neq B L T\left(C_{i} \cap C_{j}\right) \subset C_{i} \cap C_{j}$, a contradiction.

(2) $X=\cup_{i=0}^{N-1} \overline{R_{i}}$.

$X=\pi\left(\Sigma_{G}\right)=\pi \cup_{i=0}^{N-1} C_{i}=\cup_{i=0}^{N-1} \pi\left(C_{i}\right)=\cup_{i=0}^{N-1} \overline{R_{i}}$, the last equality following from Proposition 4.9.

For the next two items we need a lemma.

Lemma 6.11. Under the hypothesis of $6.10, \pi\left(\bigcap_{m}^{n} \sigma^{-k} C_{s_{k}}\right)=\overline{\bigcap_{m}^{n} \phi^{-k} R_{s_{k}}}$ for $m<n$.

Proof. Once again we use the bilaterally transitive points to deal with images of intersections. We have the following string of equalities.

$$
\pi\left(\bigcap_{m}^{n} \sigma^{-k} C_{s_{k}}\right)=\pi\left(\overline{B L T\left(\bigcap_{m}^{n} \sigma^{-k} C_{s_{k}}\right)}\right)=\overline{\pi\left(\bigcap_{m}^{n} B L T\left(\sigma^{-k} C_{s_{k}}\right)\right)}
$$

which by injectivity of $\pi$ and shift-invariance of bilateral transitive points

$$
=\overline{\bigcap_{m}^{n} \pi\left(B L T\left(\sigma^{-k} C_{s_{k}}\right)\right)}=\overline{\bigcap_{m}^{n} \pi \sigma^{-k} B L T\left(\left(C_{s_{k}}\right)\right)}
$$

which by commutativity of $\pi$ and Proposition 4.8

$$
=\overline{\bigcap_{m}^{n} \phi^{-k} \pi\left(B L T\left(C_{s_{k}}\right)\right)}=\overline{\bigcap_{m}^{n} \phi^{-k}\left(B L T \pi\left(C_{s_{k}}\right)\right)}
$$

which by Proposition 4.9

$$
\begin{aligned}
& =\overline{\bigcap_{m}^{n} \phi^{-k}\left(B L T\left(R_{s_{k}}\right)\right)}=\overline{B L T\left(\bigcap_{m}^{n} \phi^{-k}\left(R_{s_{k}}\right)\right)} \\
& =\bigcap_{m}^{n} \phi^{-k}\left(R_{s_{k}}\right) .
\end{aligned}
$$

(3) $\mathcal{R}$ is a generator. 
Because $\mathcal{C}$ is a generator, $d\left(\bigcap_{-n}^{n} \sigma^{-k} C_{s_{k}}\right) \rightarrow 0$. By Lemma $6.11, \pi\left(\bigcap_{-n}^{n} \sigma^{-k} C_{s_{k}}\right)$ $=\overline{\bigcap_{-n}^{n} \phi^{-k} R_{s_{k}}}$. So, by continuity of $\pi$, we get

$$
d\left(\overline{\bigcap_{-n}^{n} \phi^{-k} R_{s_{k}}}\right)=d\left(\bigcap_{-n}^{n} \phi^{-k} R_{s_{k}}\right) \rightarrow 0 .
$$

(4) $\mathcal{R}$ satisfies the Markov property.

Suppose $R_{s_{i}} \cap \phi^{-1} R_{s_{i+1}} \neq \emptyset, 1 \leq k \leq n-1$. By Lemma 6.11 we have $\pi\left[C_{s_{i}} \cap\right.$ $\left.\sigma^{-1} C_{s_{i+1}}\right]=\overline{R_{s_{i}} \cap \phi^{-1} R_{s_{i+1}}} \neq \emptyset, 1 \leq k \leq n-1$. Thus $C_{s_{i}} \cap \sigma^{-1} C_{s_{i+1}} \neq \emptyset, 1 \leq$ $k \leq n-1$. Since $\mathcal{C}$ satisfies the Markov property, $\bigcap_{k=1}^{n} \phi^{-k} C_{s_{k}} \neq \emptyset$ for all $n>1$. So $\pi\left(\bigcap_{k=1}^{n} \phi^{-k} C_{s_{k}}\right)=\overline{\bigcap_{k=1}^{n} \phi^{-k} R_{s_{k}}} \neq \emptyset$ for all $n>1$. Therefore, $\bigcap_{k=1}^{n} \phi^{-k} R_{s_{k}} \neq \emptyset$ for all $n>1$.

Corollary 6.12. If in addition to the hypotheses of Theorem 4.18 the dynamical system $(X, \phi)$ is expansive, then $\pi$ is finite.

Proof. We derive 4.6(iv) from the assumption that the domain of $\pi$ is irreducible, $\pi$ satisfies 4.6(i), (ii), (iii), and (v), and $\phi$ is expansive. This is an immediate consequence of Theorems 6.10 and 6.5.

Remark. We remark that a dynamical system $(X, \phi)$ which is a factor of a subshift of finite type via an essentially one-to-one finite factor map, i.e. one that satisfies properties (i)-(v) of Definition 4.6, need not be expansive (see Exercise 8.4). On the other hand D. Fried has proven $[\mathrm{F}]$.

Theorem 6.13. An expansive dynamical system $(X, \phi)$ is a factor of a subshift of finite type $\left(\Sigma_{A}, \sigma\right)$ if and only if $\phi$ has a Markov partition.

Here the factor map need not be finite. To square this with what we have proved, we note that in the "only if" part of the theorem the subshift and factor map associated with the Markov partition will not be the ones of the assumption. We further remark that here expansivity has replaced the one-to-one condition of Theorem 6.10. The proof of Fried's theorem relies on a technique of Bowen which will be mentioned in the epilogue.

\section{Exercises.}

6.1 A topological partition $\mathcal{R}$ for a dynamical system $(X, \phi)$ is Markov if and only if

$$
\bigcap_{k=0}^{n} \phi^{-k} R_{s_{k}} \neq \emptyset, \bigcap_{k=-n}^{0} \phi^{-k} R_{s_{k}} \neq \emptyset \Rightarrow \bigcap_{k=-n}^{n} \phi^{-k} R_{s_{k}} \neq \emptyset
$$

for $n \geq 0$.

6.2 Under the hypothesis of Theorem 6.5 show that if $(X, \phi)$ is irreducible, then so is $\left(\Sigma_{G}, \sigma\right)$.

6.3 Show that in Theorem 6.10 , condition (v) cannot be replaced by condition (iv). Hint: Consider the following example. Let

$$
A=\left(\begin{array}{lll}
1 & 1 & 0 \\
1 & 0 & 1 \\
1 & 1 & 0
\end{array}\right)
$$




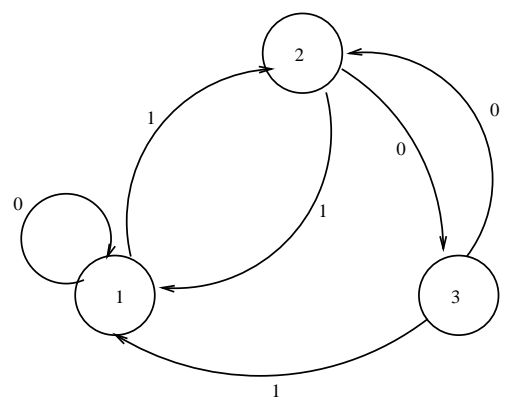

Figure 17. $\pi$ indicated by edge-labels

and $\pi: X_{\mathrm{A}} \rightarrow X_{2}$ be defined, as depicted in Figure 17, by

$$
\begin{aligned}
& \pi[1,1]=\pi[2,3]=\pi[3,2]=0 \\
& \pi[1,2]=\pi[2,1]=\pi[3,1]=1
\end{aligned}
$$

Show disjointness is violated in the image partition of the elementary cylinder sets.

6.4 Let $\pi$ be a factor map from a topological Markov shift $\left(\Sigma_{\mathrm{A}}, \sigma\right)$ to an expansive dynamical system $(X, \phi)$ with expansive constant $c$, and let the partition $\mathcal{R}$ defined by $\mathcal{R}=\left\{R_{i}=\pi\left(C_{i}\right)^{o}: i=0, \ldots, N-1\right\}$ satisfy $d(\mathcal{R})<c / 2$. Show that if $\pi$ has a diamond, then there exists a point $\mathrm{p} \in X$ with a continuum of pre-images.

\section{Product structure}

The Markov property for a topological partition is an infinite set of conditions. It is the crucial one for obtaining a topological Markov shift representation of a dynamical system, but it could be difficult to verify. However, there is another more useful criterion for getting it to which we now turn our attention. It involves exchanging one infinite set of conditions for another of a different sort that is more readily checkable. Once more we look to our concrete systems as a guide. The sets of partitions in Examples 3.1 and 3.3 have a product structure whose behavior with respect to the action of a mapping is intimately tied up with the Markov property.

A general notion of partition without regard to any other consideration is the following.

Definition 7.1. A partition of a set $R$ is defined to be a family $\mathcal{H}=\{h(\mathrm{p}): \mathrm{p} \in R\}$ of subsets of $R$ such that for $\mathrm{p}, \mathrm{q} \in R$

1. $\mathrm{p} \in h(\mathrm{p})$,

2. $h(\mathrm{p}) \cap h(\mathrm{q}) \neq \emptyset \Rightarrow h(\mathrm{p})=h(\mathrm{q})$.

Definition 7.2. We call two partitions

$$
\begin{gathered}
\mathcal{H}=\{h(\mathrm{p}): \mathrm{p} \in R\} \\
\mathcal{V}=\{v(\mathrm{p}): \mathrm{p} \in R\}
\end{gathered}
$$

of $R$ transverse if $h(\mathrm{p}) \cap v(\mathrm{q}) \neq \emptyset$ for every $\mathrm{p}, \mathrm{q} \in R$. 

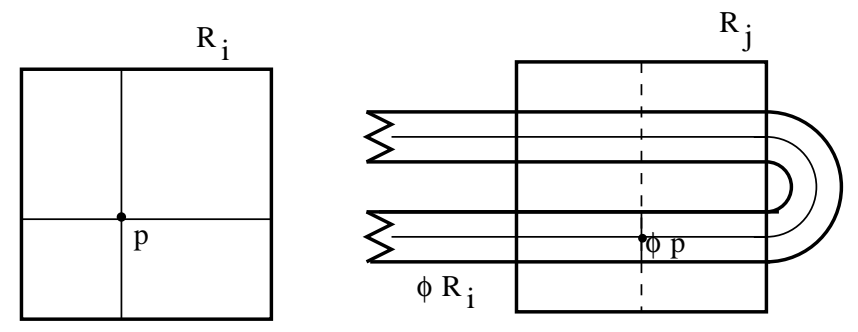

Figure 18. Property M

A set $R$ with two transverse partitions $\mathcal{H}, \mathcal{V}$ can be viewed as having a product structure something like that of a rectangle, which suggests the following graphic terminology: we shall refer to the elements $\mathcal{H}$ as horizontals and those of $\mathcal{V}$ as verticals. When we are dealing with elements $R_{i}$ of a topological partition $\mathcal{R}=$ $\left\{R_{i}: i=1, \ldots, N\right\}$, each having a pair of transverse partitions $\mathcal{H}_{i}, \mathcal{V}_{i}$, we refer to $h_{i}(\mathrm{p})$ as the horizontal through $\mathrm{p}$ in $R_{i}$ and to $v_{i}(\mathrm{p})$ as the vertical.

Next we introduce notions concerned with the behavior of horizontals and verticals under the map associated with a dynamical system. We shall stick to the convention that under the action of a map verticals seem to contract and horizontals seem to expand. While we do not insist that the diameters of the images of these sets actually increase or decrease, this will generally be the case. In fact there usually is uniform geometric expansion and contraction. In the literature one encounters the term stable set for what we call a vertical and unstable set for a horizontal.

Definition 7.3. Suppose a dynamical system $(X, \phi)$ has a topological partition $\mathcal{R}$ $=\left\{R_{i}\right\}$, each member of which has a pair of transverse partitions. We say alignment of verticals and horizontals are respectively maintained by $\phi$ and $\phi^{-1}$ if for all $i, j$

1. $\mathrm{p} \in R_{i} \cap \phi^{-1} R_{j} \Rightarrow R_{j} \cap \phi v_{i}(\mathrm{p}) \subset v_{j}(\phi \mathrm{p})$,

2. $\mathrm{p} \in R_{i} \cap \phi R_{j} \Rightarrow R_{j} \cap \phi^{-1} h_{i}(\mathrm{p}) \subset h_{j}\left(\phi^{-1} \mathrm{p}\right)$.

We actually require something stronger.

Definition 7.4. In a dynamical system $(X, \phi)$ we say a topological partition $\mathcal{R}$ $=\left\{R_{i}\right\}$ has property $M$ if each set $R_{i}$ has a pair of transverse partitions such that alignments of horizontals and verticals are maintained by $\phi$ and its inverse respectively in such a manner that the image of any vertical and the pre-image of any horizontal are contained in a unique element of $\mathcal{R}$. In other words, 7.3 (1) and (2) are replaced by:

1. $\mathrm{p} \in R_{i} \cap \phi^{-1} R_{j} \Rightarrow \phi v_{i}(\mathrm{p}) \subset v_{j}(\phi \mathrm{p})$,

2. $\mathrm{p} \in R_{i} \cap \phi R_{j} \Rightarrow \phi^{-1} h_{i}(\mathrm{p}) \subset h_{j}\left(\phi^{-1} \mathrm{p}\right)$.

See Figure 18. Also see Figure 19 for violations of alignment and property $M$.

We remark that with respect to horizontals 7.6 (2) can be expressed alternatively as follows:

$$
\mathrm{p} \in R_{i} \cap \phi^{-1} R_{j} \Rightarrow \phi h_{i}(\mathrm{p}) \supset h_{j}(\phi \mathrm{p}) .
$$

Proposition 7.5. If $\mathcal{R}$ has property $M$, then so does $\mathcal{R}^{(2)}$. (See Figure 20.) 


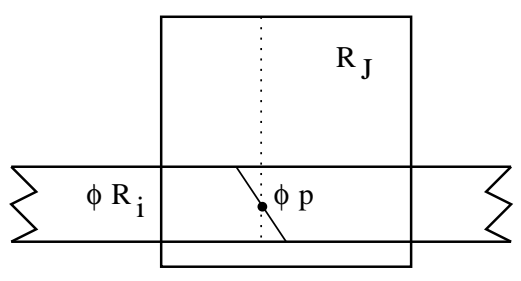

alignment

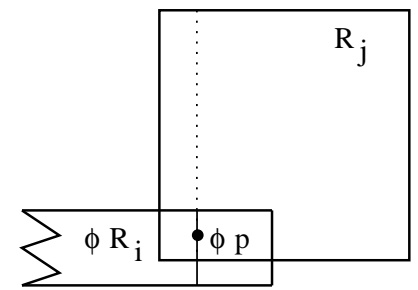

property $\mathrm{M}$

Figure 19. Violations

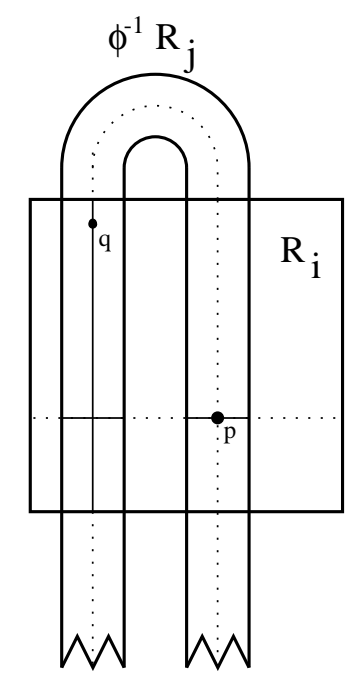

Figure 20. Property $\mathrm{M}$ on $\mathcal{R}^{(2)}$

Proof. Let $R_{i} \cap \phi^{-1} R_{j} \neq \emptyset$ be a member of $\mathcal{R}^{(2)}$. Since a partition of a set induces a partition of a subset, the horizontals and verticals of $R_{i}$ induce corresponding partitions of $R_{i} \cap \phi^{-1} R_{j}$ : namely,

1. $\mathcal{H}\left(R_{i} \cap \phi^{-1} R_{j}\right)=\left\{h_{i j}(\mathrm{p})=h_{i}(\mathrm{p}) \cap \phi^{-1} R_{j}: \mathrm{p} \in R_{i} \cap \phi^{-1} R_{j}\right\}$,

2. $\mathcal{V}\left(R_{i} \cap \phi^{-1} R_{j}\right)=\left\{v_{i j}(\mathrm{p})=v_{i}(\mathrm{p}): \mathrm{p} \in R_{i} \cap \phi^{-1} R_{j}\right\}$.

First, to verify that this pair of partitions is transverse, we observe that if $\mathrm{p}, \mathrm{q} \in$ $R_{i} \cap \phi^{-1} R_{j}$, then by $7.4(1)$

$$
v_{i}(\mathrm{q}) \subset \phi^{-1} v_{j}(\phi \mathrm{q}) \subset \phi^{-1} R_{j} .
$$

From definition (1) we have

$$
h_{i j}(\mathrm{p}) \cap v_{i j}(\mathrm{q})=h_{i}(\mathrm{p}) \cap \phi^{-1} R_{j} \cap v_{i}(\mathrm{q})=h_{i}(\mathrm{p}) \cap v_{i}(\mathrm{q}) \neq \emptyset .
$$

Second, we show that $\phi$ and its inverse map verticals and horizontals so as to satisfy property $\mathrm{M}$. Let $\mathrm{p} \in R_{i} \cap \phi^{-1} R_{j} \cap \phi^{-1}\left(R_{j} \cap \phi^{-1} R_{k}\right)$. On one hand, it is immediate from the definition that

$$
\phi v_{i j}(\mathrm{p})=\phi v_{i}(\mathrm{p}) \subset v_{j}(\phi \mathrm{p})=v_{i j}(\phi \mathrm{p}) .
$$




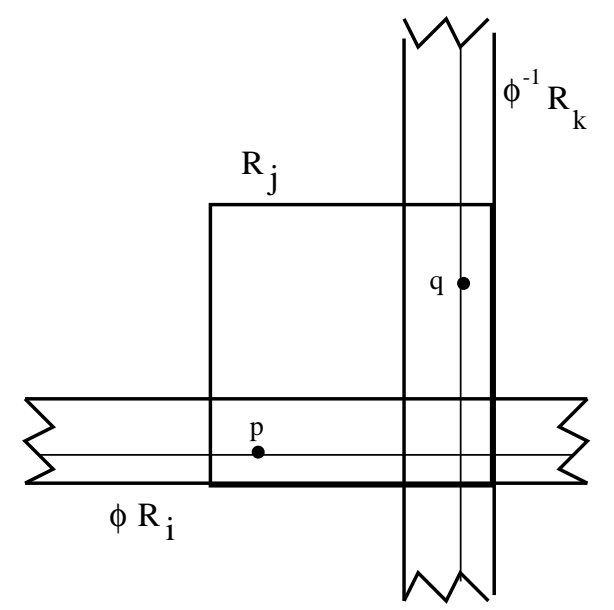

FigURE 21. 3-fold intersection property

On the other,

$$
\phi h_{i j}(\mathrm{q})=\phi\left(h_{i}(\mathrm{q}) \cap \phi^{-1} R_{j}\right) \supset h_{j}(\phi \mathrm{q}) \supset h_{j}(\phi \mathrm{q}) \cap \phi^{-1} R_{k}=h_{j k}(\phi \mathrm{q}) .
$$

Corollary 7.6. If $\mathcal{R}$ has property $M$, then so does $\mathcal{R}^{(n)}$ for $n=1,2, \ldots$.

Proof. Repeated use of Proposition 7.5 using the identity $\left(\mathcal{R}^{(n)}\right)^{(2)}=\mathcal{R}^{(n+1)}$.

Proposition 7.7. For a dynamical system $(X, \phi)$ if a topological partition $\mathcal{R}$ has property $M$, then $\mathcal{R}$ satisfies the 3-fold intersection property. (See Figure 21.)

Proof. Let $\mathrm{p} \in \phi R_{i} \cap R_{j} \neq \emptyset$ and $\mathrm{q} \in R_{j} \cap \phi^{-1} R_{k} \neq \emptyset$. Then by transversality $v_{j}(\mathrm{q}) \cap h_{j}(\mathrm{p}) \neq \emptyset$. Futhermore, $v_{j}(\mathrm{q}) \cap h_{j}(\mathrm{p}) \subset R_{j}$ and $v_{j}(\mathrm{q}) \cap h_{j}(\mathrm{p}) \subset \phi^{-1} v_{k}(\phi \mathrm{q}) \cap$ $\phi h_{i}\left(\phi^{-1} \mathrm{p}\right) \subset \phi^{-1} R_{k} \cap \phi R_{i}:$ so $\phi R_{i} \cap R_{j} \cap \phi^{-1} R_{k} \neq \emptyset$. Thus we have

$$
R_{i} \cap \phi^{-1} R_{j} \neq \emptyset, R_{j} \cap \phi^{-1} R_{k} \neq \emptyset \Rightarrow R_{i} \cap \phi^{-1} R_{j} \cap \phi^{-2} R_{k} .
$$

Corollary 7.8. Given a dynamical system $(X, \phi)$, if a topological partition $\mathcal{R}$ has property $M$, then $\mathcal{R}^{(n)}$ satisfies the 3-fold intersection property for $n=1,2, \ldots$

Proof. Follows from Corollary 7.6 and Proposition 7.7.

Theorem 7.9. Given a dynamical system $(X, \phi)$, if a topological partition $\mathcal{R}$ has property $M$, then $\mathcal{R}$ is Markov.

Proof. Follows from Corollaries 7.6, 7.8, and the Bootstrap Lemma 6.3. For instance, $\mathcal{R}^{(n-1)}$ and $\mathcal{R}^{(n)}$ satisfy the 3 -fold intersection property. So $\mathcal{R}^{(n-1)}$ satisfies the 4 -fold one. Working our way back, we get $\mathcal{R}^{(n-2)}$ satisfies the 5 -fold one, etc. Finally, we get that $\mathcal{R}$ satisfies the $(n+2)$-fold intersection property, but this is true for any $n$. 


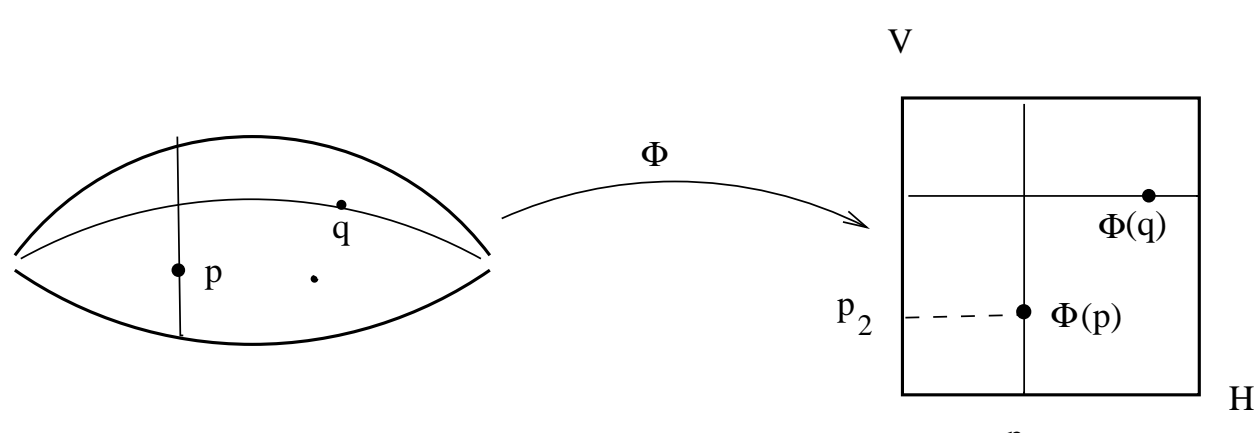

$\mathrm{p}_{1}$

FiguRE 22. Abstract rectangle

We conclude this section with a theorem which is useful in applications to dynamical systems having smooth manifolds as phase spaces. For that theorem boundaries of partition members will play a role. In addition we shall need more topological structure than that provided by mere existence of a pair of transverse partitions.

First we turn our attention to boundaries. In certain problems the burden of establishing the Markov property for a partition via property $M$ can be eased by merely verifying a similar property for boundaries. The reader will get a good illustration of this when we discuss in detail Markov partitions for automorphisms of the two torus.

Employing the usual notation, we have that the boundary of an element $R_{i}$ in a topological partition $\mathcal{R}$ is given by $\partial R_{i} \equiv \overline{R_{i}}-R_{i}$. We denote the union of all boundaries of elements of $\mathcal{R}$ by $\partial \mathcal{R} \equiv \bigcup_{i} \partial R_{i}$. Suppose the boundary $\partial R_{i}$ of each element of $\mathcal{R}$ is the union of two subsets: one, $\partial_{V} R_{i}$, which we shall call the vertical boundary of $R_{i}$, the other, $\partial_{H} R_{i}$, the horizontal boundary of $R_{i}$. We denote the union of all vertical boundaries of elements of $\mathcal{R}$ by $\partial_{V} \mathcal{R} \equiv \bigcup_{i} \partial_{V} R_{i}$, and the union of all horizontal ones by $\partial_{H} \mathcal{R} \equiv \bigcup_{i} \partial_{H} R_{i}$.

Definition 7.10. We say that a topological partition $\mathcal{R}$ has boundaries satisfying property $M$ if the following hold for each $i$ :

1. $\partial R_{i}=\partial_{V} R_{i} \cup \partial_{H} R_{i}$

2. $\overline{v_{i}(\mathrm{p})} \cap \partial R_{i} \subset \partial_{H} R_{i}$,

3. $\overline{h_{i}(\mathrm{p})} \cap \partial R_{i} \subset \partial_{V} R_{i}$,

4. $\phi \partial_{V} \mathcal{R} \subset \partial \mathcal{R}$

5. $\phi^{-1} \partial_{H} \mathcal{R} \subset \partial \mathcal{R}$.

We introduce the additional topological structure needed for the next theorem.

Definition 7.11. We call a metric space $R$ an abstract rectangle if it is homeomorphic to the Cartesian product of two metric spaces- i.e. there exist two metric spaces $H, V$ and a homeomorphism $\Phi$ of the Cartesian product $H \times V$ onto $R$. (See Figure 22.)

Sets with a pair of transverse partitions usually arise in this way. Let $\Phi\left(\mathrm{p}^{1}, \mathrm{p}^{2}\right)=$ $\mathrm{p}$, where $\mathrm{p} \in R$ and $\left(\mathrm{p}^{1}, \mathrm{p}^{2}\right) \in H \times V$. Define the following horizontal and vertical sets of $R$ :

$$
h(\mathrm{p}) \equiv \Phi\left\{\left(x, p^{2}\right): x \in H\right\}
$$



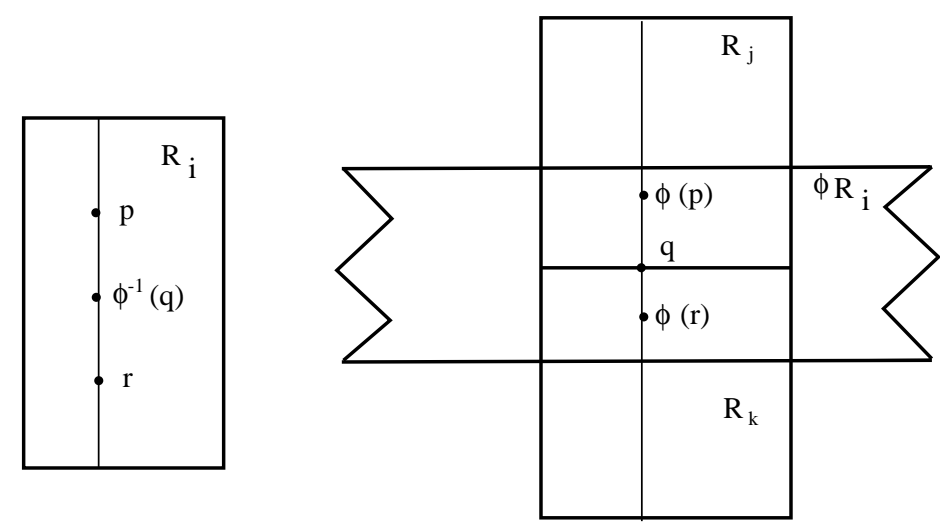

FiguRE 23. Impossible boundary picture

$$
v(\mathrm{p}) \equiv \Phi\left\{\left(p^{1}, y\right): y \in V\right\}
$$

Naturally the two partitions

$$
\begin{gathered}
\mathcal{H}=\{h(\mathrm{p}): \mathrm{p} \in R\} \\
\mathcal{V}=\{v(\mathrm{p}): \mathrm{p} \in R\}
\end{gathered}
$$

of $R$ are transverse since

$$
v(\mathrm{p}) \cap h(\mathrm{q})=\left\{\Phi\left(\mathrm{p}^{1}, \mathrm{q}^{2}\right)\right\} \neq \emptyset .
$$

In addition, for each pair of points $\mathrm{p}, \mathrm{q} \in R$ the map (p, q) $\mapsto v(\mathrm{p}) \cap h(\mathrm{q})$ is continuous, onto, and maps $h(\mathrm{p}) \times v(\mathrm{q})$ homeomorphically onto $R_{i}$. Thus we could have assumed that $H, V$ were subsets of $R$ in the first place. We designate these subsets with letters meant to suggest horizontal and vertical lines.

Theorem 7.12. In a dynamical system $(X, \phi)$, if each element of a topological partition $\mathcal{R}$ is a connected abstract rectangle, the alignments of which are maintained by $\phi$ and its inverse respectively, and if $\mathcal{R}$ has boundaries with property $M$, then $\mathcal{R}$ itself has property $M$ - i.e. $\mathcal{R}$ is a Markov partition.

Proof. We give the proof only for verticals, which consists of proving

$$
p \in R_{i} \cap \phi^{-1} R_{j} \Rightarrow \phi v_{i}(p) \subset R_{j} .
$$

See Figure 23. Our proof involves one proof by contradiction established by means of a second. The main one is a contradiction to the assumption that $\phi v_{i}(p) \nsubseteq R_{j}$. The other one contradicts the connectivity of $\phi v_{i}(p)$, which is a consequence of the following.

Since $R_{i}$ is homeomorphic to $h_{i}(p) \times v_{i}(p)$, the vertical $v_{i}(p)$ is connected: for otherwise $R_{i}$ would not be. Therefore, the homeomorphic image $\phi v_{i}(p)$ is connected as well. Thus, if $\phi v_{i}(p) \nsubseteq R_{j}$, then we would have that $\phi v_{i}(p) \cap \partial R_{j} \neq \emptyset$. Hence there would exist a point $q \in \phi v_{i}(p) \cap \partial R_{j}$ which is also in $\overline{\phi v_{i}(p) \cap R_{j}}$ : for if not, then there would be an open set $U \supset \phi v_{i}(p) \cap \partial R_{j}$ such that $U \cap \phi v_{i}(p) \cap R_{j}=\emptyset$, and the open sets $R_{j}$ and $U \cup\left(X-\overline{R_{j}}\right)$ would disconnect $\phi v_{i}(p)$. 
By preservation of alignments, we have $\phi v_{i}(p) \cap R_{j} \subset v_{j}(\phi p)$ so that $q \in \overline{v_{j}(\phi p)}$. Thus $q \in \partial_{H} \mathcal{R}$, from which follows by property $\mathrm{M}$ for boundaries that $\phi^{-1}(q) \in \partial \mathcal{R}$. However, $\phi^{-1}(q) \in v_{i}(p) \subset R_{i}$, which contradicts $R_{i} \cap \partial R_{i}=\emptyset$.

We now turn our attention to 2-dimensional toral automorphisms in generality. While there exist non-measurable automorphisms, for us toral automorphisms will mean continuous ones.

\section{MARKov PARTITIONS For AUtOMORPhisms OF THE 2-TORUS}

Let $X=\mathbb{R}^{n} / \mathbb{Z}^{n}$ be the n-dimensional torus and A a $n \times n$ matrix with integer entries and determinant \pm 1 . Such a matrix defines an automorphism of the $n$-torus in the manner described in Section 2.3. The set of such matrices forms a group called the general linear group $G L(n, \mathbb{Z})$. Both a matrix A and the automorphism $\phi$ it defines are called hyperbolic if $A$ has no eigenvalue of modulus one.

We shall devote the rest of this section to the two dimensional case; i.e. $n=2$. Let

$$
\mathrm{A}=\left(\begin{array}{ll}
a & b \\
c & d
\end{array}\right) \in G L(2, \mathbb{Z}) .
$$

Eigenvalues of $A$ are the solutions of the quadratic equation

$$
x^{2}-(\operatorname{trace} \mathrm{A}) x+\operatorname{det} \mathrm{A}=0 .
$$

Here hyperbolicity means that $A$ has two distinct eigenvalues, say $\lambda$ and $\mu$, which are irrational numbers. Since $\lambda \mu=\operatorname{det} A= \pm 1$, we can assume that $|\lambda|>1$ and $|\mu|<1$. An easy calculation shows that the row vectors

$$
\begin{aligned}
& \mathrm{v}_{\lambda}=(c, \lambda-a) \\
& \mathrm{v}_{\mu}=(c, \mu-a)
\end{aligned}
$$

are eigenvectors associated with $\lambda$ and with $\mu$ respectively. The action of $\mathrm{A}$ on a vector $\mathrm{v}$ is to contract its $\mathrm{v}_{\mu}$-component by $\mu$ and expand its $\mathrm{v}_{\lambda}$-component by $\lambda$. Directions may or may not be reversed depending on the signs of the eigenvalues. We refer to the direction of $\mathrm{v}_{\lambda}$ as the expanding direction and that of $\mathrm{v}_{\mu}$ as the contracting one. Finally, let $\ell_{\lambda}$ be a line through the origin in the expanding direction and $\ell_{\mu}$ the one in the contracting direction. See Figure 24 . We call these lines, which are invariant under the action of $\mathrm{A}$ on the plane, the expanding and contracting eigen-line respectively. The slopes of these lines are $m_{\lambda}=(\lambda-a) / c$ and $m_{\mu}=(\mu-a) / c$. From these formulae one sees that these lines pass through no lattice points other than the origin: for if they did then the slopes $m_{\lambda}$ and $m_{\mu}$ would be rational numbers and so would $\lambda$ and $\mu$.

Theorem 8.1. A hyperbolic toral automorphism is expansive.

Proof. Let $\phi$ be an automorphism and $\mathrm{p}, \mathrm{q} \in X$ be any two different points of the two dimensional torus. Let $c=|\mu| / 8$. We shall show that there exists $n \in \mathbb{Z}$ such that $d\left(\phi^{n} \mathrm{p}, \phi^{n} \mathrm{q}\right)>c$. By translation invariance of the metric we have $d(\mathrm{p}, \mathrm{q})=$ $d(\{0\}, \mathrm{p}-\mathrm{q})$ : so it suffices to show that $d\left(\{0\}, \phi^{n} \mathrm{r}\right)>c$, for any $\mathrm{r} \neq\{0\}$ in $X$.

We take the torus to be given by the fundamental region

$$
X=\{(x, y):|x| \leq 1 / 2,|y| \leq 1 / 2\}
$$




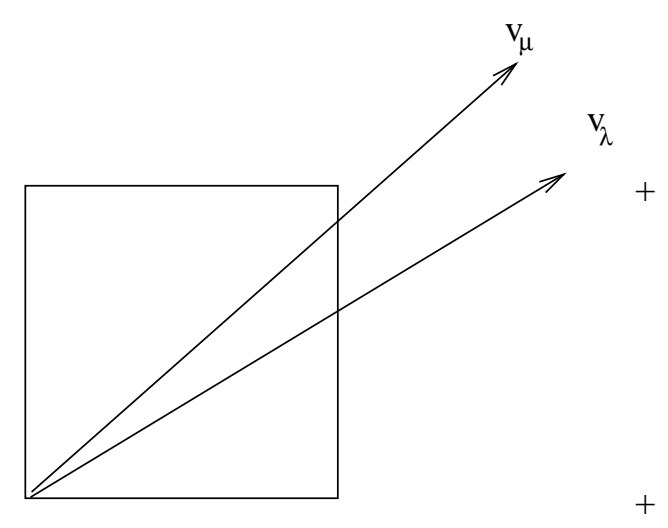

Figure 24. A fundamental region and eigen-directions of $A$

with the appropriate boundary identifications. In this region the metric on the torus coincides with the Euclidean one: namely, $d(\mathrm{p}, \mathrm{q})=\|\mathrm{p}-\mathrm{q}\|$ where $\|(x, y)\|=$ $\sqrt{x^{2}+y^{2}}$. Let $\mathrm{r}=\mathrm{p}-\mathrm{q} \neq(0,0)$. Let $\mathrm{r}_{\lambda}$ and $\mathrm{r}_{\mu}$ be the $\mathrm{v}_{\lambda}-$ component and the $\mathrm{v}_{\mu}-$ component of $\mathrm{r}$ respectively. Then from the triangle inequality

$$
\left|\lambda^{n} \mathrm{r}_{\lambda}\right|-\left|\mu^{n} \mathrm{r}_{\mu}\right| \leq\left\|\phi^{n} \mathrm{r}\right\| \leq\left|\lambda^{n} \mathrm{r}_{\lambda}\right|+\left|\mu^{n} \mathrm{r}_{\mu}\right|
$$

One of the components $r_{\lambda}, r_{\mu}$ is not zero. We can assume that $r_{\lambda} \neq 0$ : otherwise replace $\phi$ by $\phi^{-1}$ in the argument. We can also assume that $|\mu|<\frac{1}{2}$ : for, if not, replace $\phi$ by $\phi^{k}$ for large enough $k$. If $\|\mathrm{r}\|>\frac{|\mu|}{4}$, then $\left\|\phi^{n} \mathrm{r}\right\|>c$ for $n=0$. If $\|\mathrm{r}\| \leq \frac{|\mu|}{4}$, choose $n \geq 1$ such that

$$
\frac{|\mu|^{n+1}}{4} \leq\left|r_{\lambda}\right| \leq \frac{|\mu|^{n}}{4}
$$

Then the following inequalities show that $\phi^{n} \mathrm{r}$ is in the fundamental region and that $\left\|\phi^{n} \mathrm{r}\right\|>c$.

$$
\begin{gathered}
\left|\lambda^{n} \mathrm{r}_{\lambda}\right|+\left|\mu^{n} \mathrm{r}_{\mu}\right| \leq \frac{1}{4}+\frac{|\mu|^{n+1}}{4}<\frac{1}{2} \\
\frac{|\mu|}{8} \leq \frac{|\mu|}{4}-\frac{|\mu|^{n+1}}{4} \leq\left|\lambda^{n} \mathrm{r}_{\lambda}\right|-\left|\mu^{n} \mathrm{r}_{\mu}\right| .
\end{gathered}
$$

Remark. We now have all the ingredients for a formal proof that the toral automorphism of Example 3.3 enjoys the conclusions of Theorem 6.5 about representing dynamical systems by topological Markov shifts. In this case the automorphisms $\phi$ can be given by the matrix

$$
\mathrm{A}=\left(\begin{array}{ll}
1 & 1 \\
1 & 0
\end{array}\right)
$$

and the topological Markov shift $\left(\Sigma_{G}, \sigma\right)$ by the edge-graph $G$ of Figure 2. Theorem 8.1 shows that $\phi$ is expansive, a necessary item in the hypothesis of the main theorem, Theorem 6.5. Theorem 7.12 can be applied to show that the partition in Example 3.3 is Markov, another necessary item. Finally in Section 3.3 we have already shown that the partition in question is a generator, the remaining requirement of the main theorem. 
Returning our attention to the general case, we shall exploit one of the properties of a hyperbolic automorphism: namely, a matrix in the group $G L(2, \mathbb{Z})$ specifying a hyperbolic automorphism is conjugate to another one, all of whose entries bear the same sign. More specifically we have the following theorem of Williams [W], proved by entirely elementary methods of plane geometry.

Theorem 8.2. Let $\mathrm{A} \in G L(2, \mathbb{Z})$ be hyperbolic. Then there exists $\mathrm{C}, \mathrm{P} \in G L(2, \mathbb{Z})$ such that $\mathrm{CAC}^{-1}=\epsilon \mathrm{P}$ where $\epsilon= \pm 1$, the choice of sign being the same as that of $\lambda$, and the entries of the matrix

$$
\mathrm{P}=\left(\begin{array}{ll}
p & q \\
r & s
\end{array}\right)
$$

are non-negative.

Proof. Choose a pair of lattice points $(\alpha, \beta),(\gamma, \delta)$ such that

(i) the angle $(\alpha, \beta)(0,0)(\gamma, \delta)$ is acute and $\ell_{\lambda}$ lies in it, but $\ell_{\mu}$ does not;

(ii) the closed parallelogram with vertices at the origin, the two lattice points, and the lattice point $(\alpha+\gamma, \beta+\delta)$ contains no other lattice point. (See Figure 25.)

This can always be done. One way is to use continued fractions to approximate slopes of lines, a discussion of which shall be deferred to a remark. An even more elementary way is the following.

First, choose initial lattice points $(\alpha, \beta)$ and $(\gamma, \delta)$ so close to $\ell_{\lambda}$ that (i) is satisfied and no other lattice points lie between them and the origin on a direct line. Then if the parallelogram in (ii) contains another lattice point in its interior, connect it to the origin with a line segment. Form a new pair of lattice points by taking the closest lattice point to the origin on this segment and selecting the one

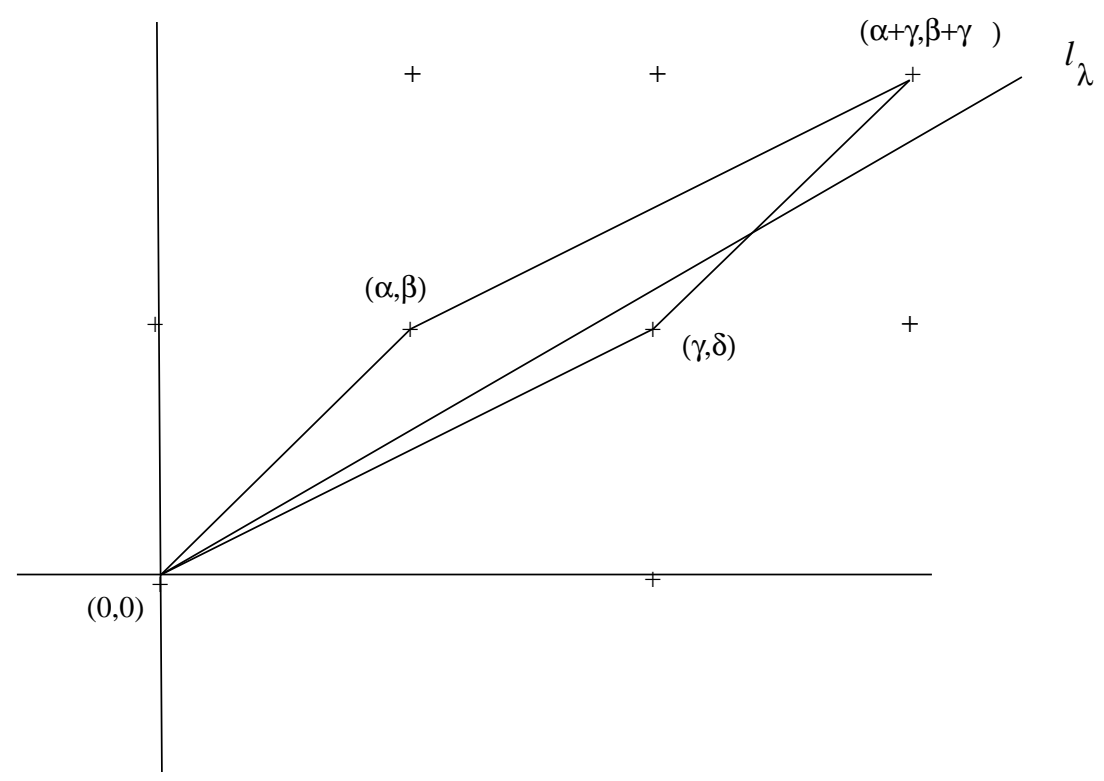

Figure 25. Parallelogram and expanding direction 
ROY L. ADLER

from the previous pair for which (i) holds. Continue this process until condition (ii) is satisfied.

Condition (ii) is equivalent to the area of the parallelogram equalling 1 .

Consider the linear map given by the matrix

$$
\mathrm{C}=\left(\begin{array}{ll}
\gamma & \delta \\
\alpha & \beta
\end{array}\right)
$$

Since

$$
\begin{aligned}
& (0,1) \mathrm{C}=(\alpha, \beta), \\
& (1,0) \mathrm{C}=(\gamma, \delta),
\end{aligned}
$$

$\mathrm{C}$ maps the principal fundamental region-namely, the closed unit square-onto the parallelogram. We shall show that $\mathrm{C}$ provides the sought-after conjugating transformation.

The two lines $\bar{\ell}_{\lambda}, \bar{\ell}_{\mu}$ that are the images under $C^{-1}$ of $\ell_{\lambda}, \ell_{\mu}$ are the expanding and contracting eigen-lines for each of the transformations $\pm \mathrm{CAC}^{-1}$. Choose $P$ to be the one that preserves the orientation of $\bar{\ell}_{\lambda}$, the choice of sign being that of $\lambda$. This means that the matrix $\mathrm{P}$ has $|\lambda|$ as the expanding eigenvalue and either $\pm \mu$ as the contracting. Furthermore, because $\mathrm{C}^{-1}$ maps the parallelogram onto the unit square and $\ell_{\lambda}$ passes through the parallelogram while $\ell_{\mu}$ does not, the line $\bar{\ell}_{\lambda}$ passes through the first quadrant and the line $\bar{\ell}_{\mu}$ the second.

To prove that $\mathrm{P}$ is non-negative, or equivalently, that $\mathrm{P}$ maps the first quadrant into itself, we must just show that $(0,1) \mathrm{P}$ and $(1,0) \mathrm{P}$ lie in the first quadrant. We shall give the proof only for $(0,1) \mathrm{P}$ : the arguments which follow work equally well for the other lattice point $(1,0) \mathrm{P}$.

There are two cases depending on whether the contracting eigenvalue $\mu$ of $\mathrm{P}$ is positive or negative. In the second case $(\mu<0)$, the linear map $\mathrm{P}$ reflects the first quadrant about the eigen-line $\bar{\ell}_{\lambda}$, while in the first case $(\mu>0)$ no reflection takes place. Let $\mathrm{c}$ denote the origin, a the lattice point $(0,1)$, and $\mathrm{a}^{\prime}$ its image under $P$. Let $\mathrm{b}$ be the projection of a on the line $\bar{\ell}_{\lambda}$ in the direction parallel to $\bar{\ell}_{\mu}$ and $\mathrm{b}^{\prime}$ its image under P. See Figure 26. The point $\mathrm{b}^{\prime}$ is also the projection of $\mathrm{a}^{\prime}$ on the line $\bar{\ell}_{\lambda}$.

We deal first with the case without reflection. Let $\bar{\ell}_{\lambda}^{\prime}, \bar{\ell}_{\mu}^{\prime}$ be the lines through $a$ parallel to $\bar{\ell}_{\lambda}, \bar{\ell}_{\mu}$ respectively. The notation $|\mathrm{pq}|$ stands for the length of the line segment with end-points p, q. On one hand, since $\left|a^{\prime} b^{\prime}\right|=|\mu|^{-1} \cdot|a b|<|a b|$, the point $\mathrm{a}^{\prime}$ lies between the lines $\bar{\ell}_{\lambda}^{\prime}, \bar{\ell}_{\lambda}$. On the other, since $\left|\mathrm{cb}^{\prime}\right|=|\lambda| \cdot|\mathrm{cb}|>|\mathrm{cb}|$, the point $a^{\prime}$ lies to the left of $\bar{\ell}_{\mu}^{\prime}$. The region bounded by these three lines, in which $a^{\prime}$ thus lies, is contained in the first quadrant.

For the case with reflection, let $c^{\prime}$ be the intersection of the line $\bar{\ell}_{\lambda}$ and the vertical through $a^{\prime}$. See Figure 27. Suppose that $a^{\prime}$ belongs to the fourth quadrant but not the first. The point $a^{\prime}$ being a lattice point implies that $\left|\mathrm{a}^{\prime} \mathrm{c}^{\prime}\right|>1$. However, because triangle $\mathrm{c}^{\prime} \mathrm{a}^{\prime} \mathrm{b}^{\prime}$ is similar to cab, $\left|\mathrm{a}^{\prime} \mathrm{c}^{\prime}\right|=|\mu| \cdot|\mathrm{ac}|<|\mathrm{ac}|<1$, a contradiction.

Remark. By means of continued fractions we can somewhat augment the conclusion of Theorem 8.2: namely, we can conjugate so that the following two conditions hold simultaneously.

1. $\bar{m}_{\mu}<-1$, 


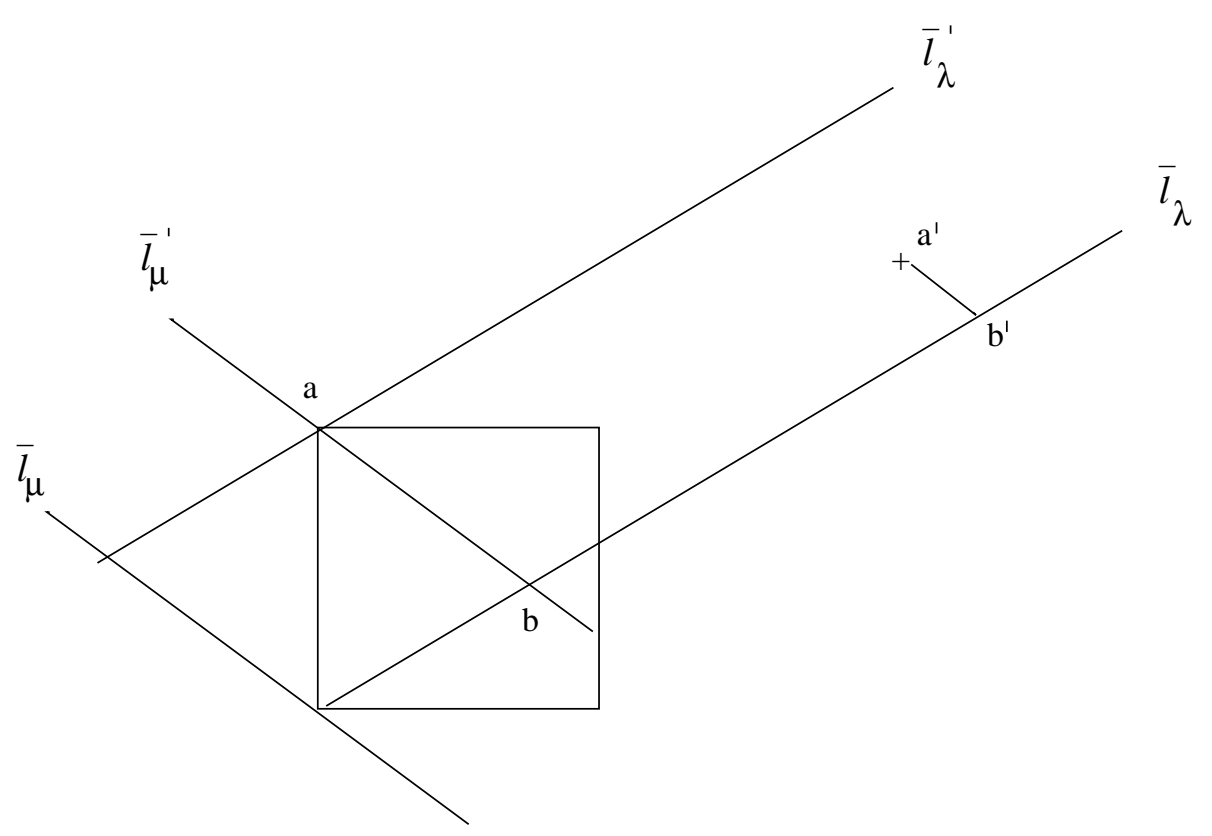

FiguRE 26. Geometrical figure for proof without reflection

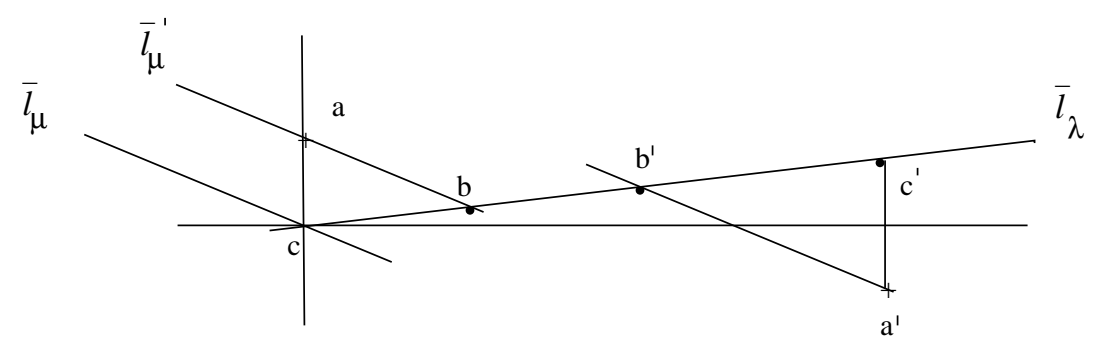

FiguRE 27. Geometrical figure for proof with reflection

2. $0<\bar{m}_{\lambda}<1$,

where $\bar{m}_{\mu}$ is the slope of the contracting eigen-line $\bar{\ell}_{\mu}$ and $\bar{m}_{\lambda}$ is the slope of the expanding one $\bar{\ell}_{\lambda}$ for $\mathrm{P}$.

The first inequality indicates that the contracting eigen-line for $\mathrm{P}$ passes through the second quadrant between the lattice points $(0,1)$ and $(-1,1)$, and the second that the expanding one passes through the first quadrant under the lattice point $(1,1)$. For Theorem 8.4, the main one of this section, one does not need more than what is provided by Theorem 8.2. These extra properties make life a little less difficult. The first one makes a key figure, Figure 29 on page 42, easier to draw. The second one obviates repeating proofs covering slightly different geometrical figures. Not taking advantage of it multiplies the number of cases in the proof, and we shall have enough of them as it is. Since we shall not be using the full strength of this remark, one can skip the remainder of it and proceed directly to Theorem 8.4. We shall be using the second property, which is very easy to achieve by itself. 
ROY L. ADLER

From the theory of continued fractions, we know that every irrational number can be written uniquely as an infinite continued fraction $\left[a_{0}, a_{1}, \ldots\right]=a_{0}+1 /\left(a_{1}+\right.$ $1 / \ldots)$ where $a_{n} \in \mathbb{Z}$ for all $n$ and $a_{n}>0$ for $n>0$. In addition, the continued fraction of a quadratic surd has a periodic tail: namely, the tail can be written as $\left[\overline{b_{1}, \ldots, b_{m}}\right]>1$, where the overbar means infinite repetition of $b_{1}$ through $b_{m}$. In [ATW] the following was proved.

Theorem 8.3. Let $\mathrm{A} \in G L(2, \mathbb{Z})$ be hyperbolic. The slope $m_{\lambda}$, being a quadratic surd, can be written $m_{\lambda}=\left[a_{0}, a_{1}, \ldots, a_{n}, \overline{b_{1}, \ldots, b_{m}}\right]$, where $m$ is as small as possible. If

$$
\mathrm{C}=\left(\begin{array}{cc}
0 & 1 \\
1 & a_{0}
\end{array}\right)\left(\begin{array}{cc}
0 & 1 \\
1 & a_{1}
\end{array}\right) \cdots\left(\begin{array}{cc}
0 & 1 \\
1 & a_{n}
\end{array}\right)
$$

then $\mathrm{CAC}^{-1}=\epsilon \mathrm{P}$ where

$$
\mathrm{P}=\left(\left(\begin{array}{cc}
0 & 1 \\
1 & b_{1}
\end{array}\right) \ldots\left(\begin{array}{cc}
0 & 1 \\
1 & b_{m}
\end{array}\right)\right)^{N}
$$

for some positive integer $N$ and $\epsilon$ is the same as in Theorem 8.2. Furthermore, the slopes of the eigen-lines of $\mathrm{P}$ satisfy $\bar{m}_{\lambda}=\left[\overline{b_{1}, \ldots, b_{m}}\right]>1$ and $-1 / \bar{m}_{\mu}=$ $\left[\overline{b_{m}, \ldots, b_{1}}\right]>1$.

The matrix

$$
\left(\begin{array}{ll}
0 & 1 \\
1 & 0
\end{array}\right) P\left(\begin{array}{ll}
0 & 1 \\
1 & 0
\end{array}\right)
$$

achieves the above two conditions in the remark.

Theorem 8.4. Let $\phi$ be a toral automorphism whose defining matrix is either $\mathrm{P}$ or $-\mathrm{P}$ where

$$
\mathrm{P}=\left(\begin{array}{ll}
p & q \\
r & s
\end{array}\right)
$$

is a hyperbolic matrix in $G L(2, \mathbb{Z})$ with non-negative entries. Then there exists a Markov generator $\mathcal{R}^{*}$ for $\phi$, the members of which are parallelograms. The associated Markov shift is given by a directed graph also specified by $\mathrm{P}$ : i.e., the edge graph with connections given by $\mathrm{P}$ consists of two vertices labelled I and II with $p$ directed edges from I to itself, $q$ from I to II, $r$ from II to I, and $s$ from II to itself. See Figure 28.

Proof. We shall assume that the expanding eigen-line of $\mathrm{P}$, the matrix given by Theorem 8.2 , passes under the point $(1,1)$; if not, conjugate $\mathrm{P}$ by the matrix

$$
\mathrm{E}=\left(\begin{array}{ll}
0 & 1 \\
1 & 0
\end{array}\right)
$$

which reflects the first quadrant about the line $y=x$.

Before proceeding in earnest, we need some notation. Dropping the bars, we now let $\mathrm{v}_{\lambda}, \mathrm{v}_{\mu}$ be the expanding and contracting eigen-vectors of $\mathrm{P}$ and $\ell_{\lambda}, \ell_{\mu}$ the corresponding eigen-lines through the origin. We denote lines parallel to these through a point $\mathrm{p}$ by assigning $\mathrm{p}$ as a superscript. For example, $\ell_{\lambda}^{(0,1)}$ denotes the line through $(0,1)$ parallel to $\ell_{\lambda}$, etc. 


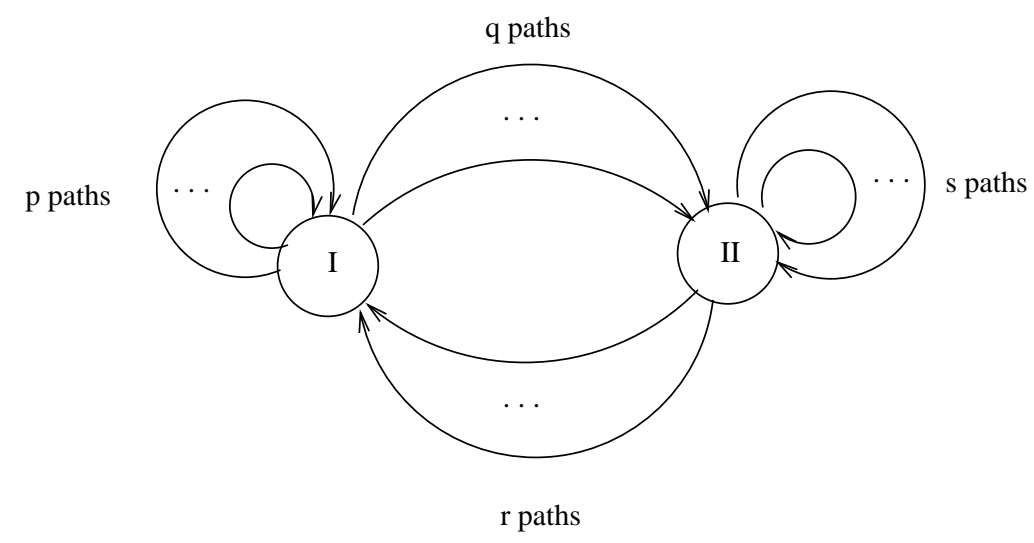

Figure 28. Edge graph defined by $\mathrm{P}$

We define the following points as depicted in Figure 29:

$$
\begin{aligned}
\mathrm{o} & \equiv(0,0) \\
\mathrm{o}^{\prime} & \equiv(1,0) \\
\mathrm{o}^{\prime \prime} & \equiv(1,1) \\
\mathrm{o}^{\prime \prime \prime} & \equiv(0,1) \\
\mathrm{a} & \equiv \ell_{\mu} \cap \ell_{\lambda} \\
\mathrm{a}^{\prime} & \equiv \ell_{\mu}^{(1,0)} \cap \ell_{\lambda}^{(1,0)} \\
\mathrm{a}^{\prime \prime} & \equiv \ell_{\mu}^{(1,1)} \cap \ell_{\lambda}^{(1,1)} \\
\mathrm{a}^{\prime \prime \prime} & \equiv \ell_{\mu}^{(0,1)} \cap \ell_{\lambda}^{(0,1)} \\
\mathrm{b} & \equiv \ell_{\mu} \cap \ell_{\lambda}^{(-1,0)} \\
\mathrm{b}^{\prime} & \equiv \ell_{\mu}^{(1,0)} \cap \ell_{\lambda}=b+(1,0) \\
\mathrm{b}^{\prime \prime} & \equiv \ell_{\mu}^{(1,1)} \cap \ell_{\lambda}^{(0,1)}=\mathrm{b}+(1,1) \\
\mathrm{c} & \equiv \ell_{\mu} \cap \ell_{\lambda}^{(0,1)} \\
\mathrm{c}^{\prime} & \equiv \ell_{\mu}^{(1,0)} \cap \ell_{\lambda}^{(1,1)} \\
\overline{\mathrm{c}} & \equiv \ell_{\mu}^{(0,-1)} \cap \ell_{\lambda} \\
\overline{\mathrm{d}} & \equiv \ell_{\mu} \cap \ell_{\lambda}^{(-1,1)} \\
\mathrm{d}^{\prime} & \equiv \ell_{\mu}^{(1,0)} \cap \ell_{\lambda}^{(0,1)}=\overline{\mathrm{d}}+(1,0) \\
\mathrm{d}^{*} & \equiv \mathrm{d}^{\prime}-(0,1) .
\end{aligned}
$$

We have drawn Figure 29 as if the first statement in the remark following Theorem 8.2 holds. This places the point $\mathrm{c}^{\prime}$ in the unit square. Since we are not using this condition, $\mathrm{c}^{\prime}$ could appear anywhere to the left of the line $x=1$ in the strip between the lines $y=0$ and $y=1$ and above the line $y=x$.

Let $R_{I}$ be the interior of parallelogram acd' $\mathrm{b}^{\prime}$ and $R_{I I}$ the interior of parallelogram $c^{\prime} d^{\prime} b^{\prime \prime} a^{\prime \prime}$. 
ROY L. ADLER

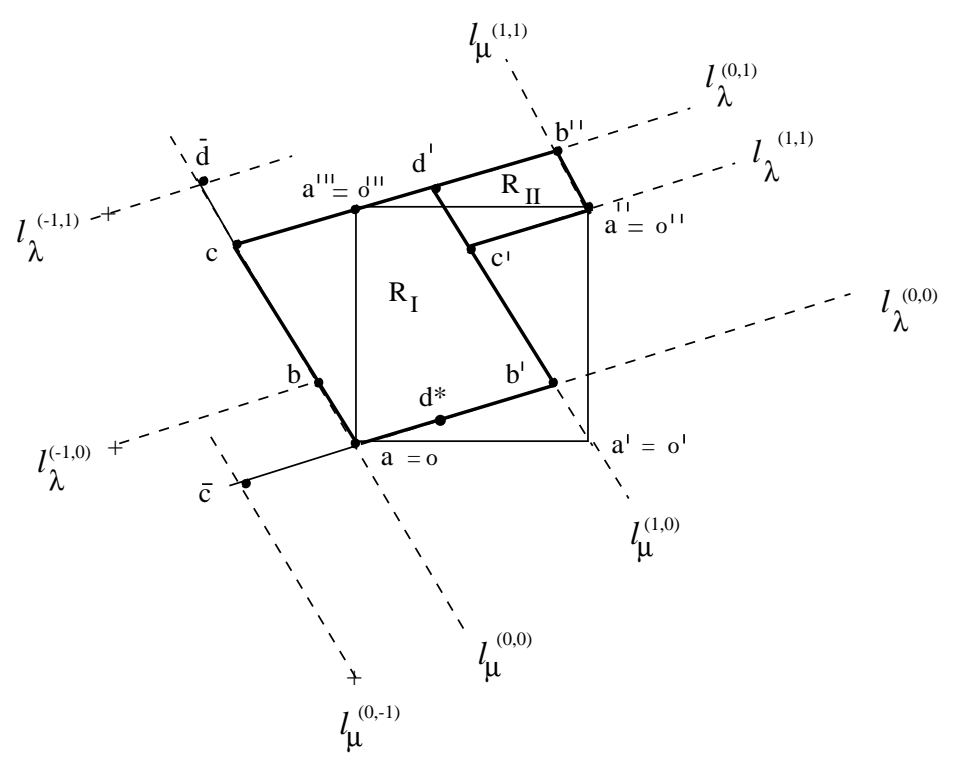

FiguRE 29. A remarkable fundamental region

The closed set $\overline{R_{I}} \cup \overline{R_{I I}}$, as we shall show, is a fundamental region, which we shall call the principal Markov one. As drawn in Figure 29, this set is equivalent modulo $\mathbb{Z}^{2}$ to the unit square by sliding $\triangle \mathrm{aa}^{\prime \prime \prime} \mathrm{c}$ one unit to the right and $\triangle \mathrm{a}^{\prime \prime \prime} \mathrm{b}^{\prime \prime} \mathrm{a}^{\prime \prime}$ one unit down. But in general we need a slightly more elaborate proof.

First, no two points in the interior of $\overline{R_{I}} \cup \overline{R_{I I}}$ are equivalent because $R_{I} \cup R_{I I}$ is disjoint from its four neighboring unit translates which totally bound it. Second, the set of all $\mathbb{Z}^{2}$-translates of $\overline{R_{I}} \cup \overline{R_{I I}}$ covers the plane because all integral horizontal translates of the union of this set with its unit downward vertical translate covers the infinite strip between the lines $y=0$ and $y=1$ and all integral vertical translates of the strip cover the plane.

Thus we can view the torus to be the set $X=\overline{R_{I}} \cup \overline{R_{I I}}$ with points on the boundary identified by lattice translations.

The proof of the theorem involves four cases: two subcases arise for each matrix $\pm \mathrm{P}$ depending on whether $\mu$ is positive or negative. These amount to the four possible combinations,

$$
(\lambda, \mu)=( \pm|\lambda|, \pm|\mu|),
$$

of signs for the eigenvalues of the matrix representing $\phi$. The simplest case is when both $\lambda$ and $\mu$ are positive. Things are more difficult when either is negative, especially $\mu$. So that the proof appears less tedious, we divide each case into five steps.

Case I: $\lambda>0, \mu>0$.

Step 1. The family $\mathcal{R}=\left\{R_{I}, R_{I I}\right\}$ is a Markov partition.

Our aim is to show that $\mathcal{R}$ satisfies the hypothesis of Theorem 7.12.

By virtue of their construction as parallelograms the members $R_{I}, R_{I I}$ of $\mathcal{R}$ are connected. These open parallelograms are obviously abstract rectangles, each being homeomorphic to the Cartesian product of two open intervals. The two in question 
are a pair of intersecting sides of a parallelogram minus endpoints. Horizontals and verticals of $R_{i}, i=I, I I$, are given by

$$
\begin{aligned}
& v_{i}(\mathrm{p}) \equiv \ell_{\mu}^{\mathrm{p}} \cap R_{i}, \\
& h_{i}(\mathrm{p}) \equiv \ell_{\lambda}^{\mathrm{p}} \cap R_{i} .
\end{aligned}
$$

Since $\mathrm{P}$ defines a linear transformation of the plane, the image of a line parallel to an eigen-line is another line parallel to the same eigen-line: so alignment of verticals and horizontals is maintained by $\phi$ and $\phi^{-1}$ : namely,

$$
\begin{aligned}
& \mathrm{p} \in R_{i} \cap \phi^{-1} R_{j} \Rightarrow R_{j} \cap \phi v_{i}(\mathrm{p}) \subset v_{j}(\phi p), \\
& \mathrm{p} \in R_{i} \cap \phi R_{j} \quad \Rightarrow R_{j} \cap \phi^{-1} h_{i}(\mathrm{p}) \subset h_{j}\left(\phi^{-1} p\right)
\end{aligned}
$$

for $i, j=I, I I$.

Next we verify that the boundaries of these members satisfy property $M$ of Definition 7.10 which entails five items. The first of these concerns dividing $\partial R_{I}$ and $\partial R_{I I}$ into vertical and horizontal pieces. As shown in Figure 29 we have

$$
\begin{gathered}
\partial R_{I}=\partial_{V} R_{I} \cup \partial_{H} R_{I}, \\
\partial R_{I I}=\partial_{V} R_{I I} \cup \partial_{H} R_{I I},
\end{gathered}
$$

where

$$
\begin{aligned}
\partial_{V} R_{I} & \equiv \mathrm{ac} \cup \mathrm{b}^{\prime} \mathrm{d}^{\prime}, \\
\partial_{H} R_{I} & \equiv \mathrm{ab}^{\prime} \cup \mathrm{cd}^{\prime}, \\
\partial_{V} R_{I I} & \equiv \mathrm{c}^{\prime} \mathrm{d}^{\prime} \cup \mathrm{a}^{\prime \prime} \mathrm{b}^{\prime \prime}, \\
\partial_{H} R_{I I} & \equiv \mathrm{c}^{\prime} \mathrm{a}^{\prime \prime} \cup \mathrm{d}^{\prime} \mathrm{b}^{\prime \prime},
\end{aligned}
$$

each being the union of two line segments. Utilizing the boundary identifications, we have that $\partial \mathcal{R}$ consists of two transverse line segments intersecting at the origin: namely,

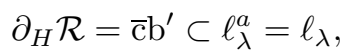

$$
\begin{aligned}
& \partial_{V} \mathcal{R}=\operatorname{ad} \subset \ell_{\mu} .
\end{aligned}
$$

It is clear from Figure 29 that (2) and (3) of 7.10 are satisfied: namely,

$$
\begin{aligned}
& \overline{v_{i}(\mathrm{p})} \cap \partial R_{i} \subset \partial_{H} R_{i}, \\
& \overline{h_{i}(\mathrm{p})} \cap \partial R_{i} \subset \partial_{V} R_{i} .
\end{aligned}
$$

From (8.6) and the property that the restrictions of $\mathrm{P}$ and $\mathrm{P}^{-1}$ respectively to the lines $\ell_{\lambda}$ and $\ell_{\mu}$ are contractions we get

$$
\begin{aligned}
\overline{\mathrm{c}} \mathrm{b}^{\prime} & \subset\left(\overline{\mathrm{c}} \mathrm{b}^{\prime}\right) P \\
(\mathrm{a} \overline{\mathrm{d}}) P & \subset \mathrm{a} \overline{\mathrm{d}} .
\end{aligned}
$$

We can restate (8.7) as items (4) and (5) of 7.10: namely,

$$
\begin{array}{r}
\phi \partial_{V} \mathcal{R} \subset \partial \mathcal{R}_{V} \subset \partial \mathcal{R}, \\
\phi^{-1} \partial_{H} \mathcal{R} \subset \partial_{H} \mathcal{R} \subset \partial \mathcal{R} .
\end{array}
$$

Thus we have established that $\mathcal{R}$ satisfies the hypothesis of Theorem 7.12: so it is a Markov partition. 
Remark. At this point it may be instructive to remark on our definition of topological partition. This example illustrates the advantage of using open sets over their closures as members of such a partition. For one thing, $R_{I} \neq{\overline{R_{I}}}^{\circ}$ and $R_{I I} \neq{\overline{R_{I I}}}^{\circ}$. For another, while $R_{I}$ and $R_{I I}$ are abstract rectangles, their closures are not. For instance, $R_{I}$ is homeomorphic to a Cartesian product: namely, the product of the line segment $\mathrm{ab}^{\prime}$ minus the endpoints with the segment $a c$ minus the endpoints. However, $\overline{R_{I}}$ is not homeomorphic to the Cartesian product of ab' with ac, since part of the segment $\mathrm{ab}^{\prime}$ is in the boundary and part is not. Furthermore, $\partial_{V} \mathcal{R}$ and $\partial_{H} \mathcal{R}$ are connected line segments which get mapped into themselves under $\phi$ and $\phi^{-1}$ respectively. This would not be the case if $\mathcal{R}=\left\{\overline{R_{I}}, \overline{R_{I I}}\right\}$. If one's definition of topological partition involves closures of open sets as members rather than open sets, then one is forced into somewhat greater contortions in order to achieve the same results.

The Markov partition $\mathcal{R}$ is not necessarily a generator. The trouble is that, while the members of $\mathcal{R}$ are connected, those of $\mathcal{R}^{(2)}=\mathcal{R} \vee \phi^{-1} \mathcal{R}$ may not be, in which case non-empty sets of the form $\bigcap_{n=0}^{\infty} \overline{\bigcap_{-n}^{n} \phi^{-k} R_{s_{k}}}$ may consist of more than one point. To overcome this let us examine the sets $R_{i} \cap \phi^{-1} R_{j} \in \mathcal{R} \vee \phi^{-1} \mathcal{R}$. As we shall see each consists of a union of disjoint open parallelograms, the number of which is given by the matrix $P$. A remedy is immediately suggested.

Step 2. The family $\mathcal{R}^{*}=\left\{R_{k}^{*}: 1 \leq k \leq N^{*}\right\}$ consisting of all connected components of the sets $R_{i} \cap \phi^{-1} R_{j} \in \mathcal{R} \vee \phi^{-1} \overline{\mathcal{R}}$ is a Markov partition.

Once again we must show that the members of $\mathcal{R}^{*}$ satisfy the five items of Definition 7.10.

In the universal cover the image $\left(R_{i}\right) \mathrm{P}$ is an open parallelogram that has been stretched by a factor of $\lambda$ in the $v_{\lambda}$-direction and shrunk by a factor $\mu$ in the $v_{\mu^{-}}$ direction. This parallelogram passes through various Markov fundamental regions, and in each one when it intersects a parallelogram equivalent to $R_{j}$, the intersection is a parallelogram. No two of these intersections share an equivalent point: for otherwise a violation of 2.1.1(3) with respect to the fundamental region $\left(\overline{R_{I}} \cup \overline{R_{I I}}\right) \mathrm{P}$ would be committed. While the set $\phi h_{i}(\mathrm{p}) \cap R_{j}$ may consist of several horizontals, the set $\phi h_{i}(\mathrm{p}) \cap R_{k}^{*}$ is either empty or a single one. Furthermore, if $\phi h_{i}(\mathrm{p}) \cap R_{k}^{*} \neq \emptyset$ for one horizontal $h_{i}(\mathrm{p})$ of $R_{i}$, then $\phi h_{i}\left(\mathrm{p}^{\prime}\right) \cap R_{k}^{*} \neq \emptyset$ for any other horizontal $h_{i}\left(\mathrm{p}^{\prime}\right)$ in $R_{i}$. Back on the torus the various non-equivalent parallelograms represent disjoint connected sets which we have labelled $R_{k}^{*}, 1 \leq k \leq N^{*}$. Being parallelograms, these sets are abstract rectangles.

Alignment of verticals and horizontals for these members of $\mathcal{R}^{*}$ is maintained by $\phi$ and $\phi^{-1}$ for the same reason it is for members of $\mathcal{R}$.

Regarding boundaries we have that

$$
\begin{aligned}
& \partial_{H} \mathcal{R}^{(2)}=\partial_{H} \mathcal{R}^{*}=\left(\overline{\mathrm{c}} \mathrm{b}^{\prime}\right) \mathrm{P} \\
& \partial_{V} \mathcal{R}^{(2)}=\partial_{V} \mathcal{R}^{*}=\mathrm{a} \overline{\mathrm{d}} .
\end{aligned}
$$

Thus, just as for $\mathcal{R}$, we have

$$
\begin{aligned}
& \phi \partial_{V} \mathcal{R}^{*}=(\mathrm{a} \overline{\mathrm{d}}) P \subset \mathrm{a} \overline{\mathrm{d}}=\partial \mathcal{R}_{V}^{*} \subset \partial \mathcal{R}^{*},
\end{aligned}
$$

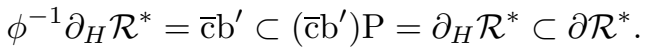

The partition $\mathcal{R}^{*}$ satisfies the hypothesis of Theorem 7.12: so it is Markov. By reasoning as before, a parallelogram of the form $\left(R_{k}^{*}\right) \mathrm{P}$ passes through various 


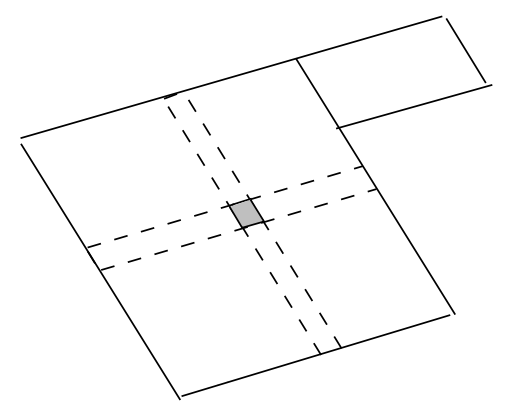

FIGURE 30. A non-empty parallelogram of the form $\bigcap_{-n}^{n} \phi^{-k} R_{s_{k}}^{*}$

fundamental regions; and in each one when it intersects a parallelogram equivalent to $R_{l}^{*}$, the intersection is a parallelogram, and no two of these intersections share an equivalent point. Hence, a non-empty set of the form $\phi R_{i}^{*} \cap R_{j}^{*}$ is a single connected parallelogram.

Step 3. If $R_{k}^{*}$ is one of the parallelograms in $\phi R_{i} \cap R_{j}$, then the length of its $v_{\lambda^{-}}$ dimension is the same as that of $R_{j}$, while the length of its $\mathrm{v}_{\mu}$-dimension is $|\mu|$ times that of $R_{i}$. Similarly, a non-empty set of the form $\phi R_{i}^{*} \cap R_{j}^{*}$ is a single connected open parallelogram, the length of its $\mathrm{v}_{\lambda}$-dimension being the same as that of $R_{j}^{*}$ and the length of its $\mathrm{v}_{\mu}$-dimension $\mu$ times that of $R_{i}^{*}$.

Likewise, a non-empty set of the form $\bigcap_{-n}^{n} \phi^{-k} R_{s_{k}}^{*}$ is a single connected open parallelogram (as depicted by the shaded area of Figure 30), the length of its $\mathrm{v}_{\lambda^{-}}$ dimension being $|\mu|^{n}$ times that of $R_{s_{-n}}^{*}$ and the length of its $\mathrm{v}_{\mu}$-dimension $|\mu|^{n}$ times that of $R_{s_{n}}^{*}$. From this we have

$$
d\left(\bigvee_{-n}^{n} \phi^{k} \mathcal{R}^{*}\right)=d(\mathcal{R}) /|\mu|^{n+1} \rightarrow 0, \text { as } n \rightarrow \infty
$$

In other words, $\mathcal{R}^{*}$ is a generator.

Step 4. Let $\#\left(\phi R_{i} \cap R_{j}\right)$ denote the number of disjoint parallelograms in the intersection $\phi R_{i} \cap R_{j}$.

$$
\begin{aligned}
\#\left(\phi R_{i} \cap R_{I}\right) & \equiv \text { number of lines } x=0, x=1, \ldots, \text { traversed by }\left(R_{i}\right) P \\
\#\left(\phi R_{i} \cap R_{I I}\right) & \equiv \text { number of lines } y=1, y=2, \ldots, \text { traversed by }\left(R_{i}\right) P,
\end{aligned}
$$

which leads to

$$
\begin{aligned}
\#\left(\phi R_{I} \cap R_{I}\right) & =x \text {-coordinate of }(0,1) \mathrm{P}=p ; \\
\#\left(\phi R_{I I} \cap R_{I}\right) & =x \text {-coordinate of }(1,1) \mathrm{P}-(1,0) \mathrm{P}=q ; \\
\#\left(\phi R_{I} \cap R_{I I}\right) & =y \text {-coordinate of }(0,1) \mathrm{P}=r ; \\
\#\left(\phi R_{I I} \cap R_{I I}\right) & =y \text {-coordinate of }(1,1) \mathrm{P}-(1,0) \mathrm{P}=s
\end{aligned}
$$

To establish Step 4 it is important to understand how $\left(R_{I}\right) P$ and $\left(R_{I I}\right) P$ intersect various Markov fundamental regions, particularly how they begin and end. We must show that Figure 31 truly represents the situation: namely, the segment $\left(b^{\prime} d^{\prime}\right) \mathrm{P}$ lies within the segment $\tilde{a} \tilde{b}$. First, if a parallelogram $\left(R_{i}\right) \mathrm{P}$ intersects a lattice translate of $R_{j}$, then it stripes all the way across. As it does do, it passes strictly 


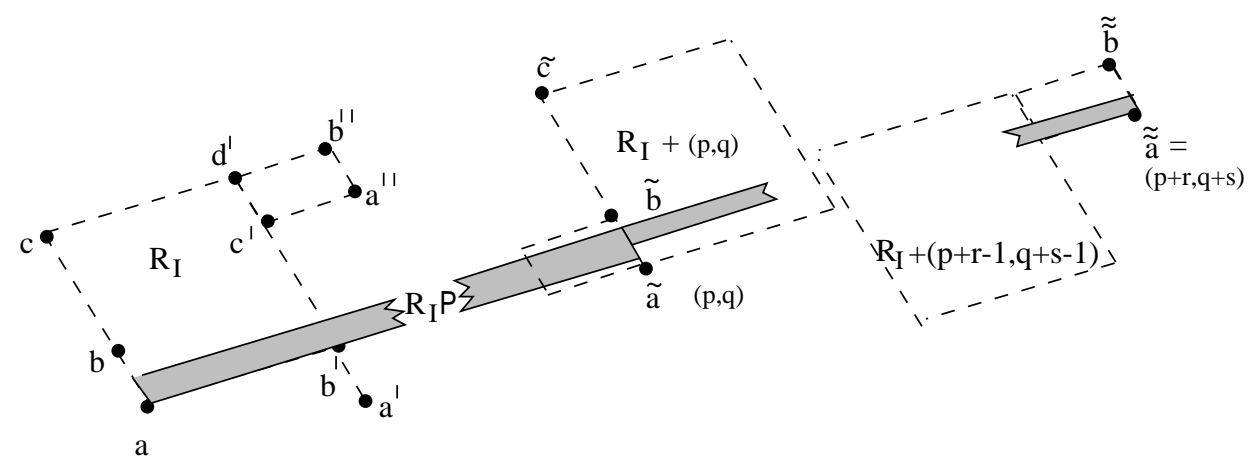

Figure 31. How $\left(R_{I}\right) \mathrm{P}$ and $\left(R_{I I}\right) \mathrm{P}$ intersect various fundamental regions

through the lattice translate without ever straddling any part of a horizontal boundary: for otherwise there would be a violation of the property that $\left(\overline{R_{I}} \cup \overline{R_{I I}}\right) \mathrm{P}$ is a fundamental region because $\partial_{H}(\mathcal{R P}) \supset \partial_{H} \mathcal{R}$.

Since $\mathrm{P}$ is a contraction on the line $\ell_{\mu}$, we have $|(\mathrm{ab}) \mathrm{P}|<|\mathrm{ab}|$; and since the point $\mathrm{a}=(0,0)$ is fixed, the point $(\mathrm{b}) \mathrm{P}$ lies inside the segment ab. It then follows that the point $\left(\mathrm{b}^{\prime}\right) \mathrm{P}=(\mathrm{b}) \mathrm{P}+(p, q)$ lies inside a lattice translate of ab-that is, it lies on the line $\ell_{\mu}^{(p, q)}$ strictly between $\tilde{\mathrm{a}} \equiv(p, q)=\left(\mathrm{a}^{\prime}\right) \mathrm{P}$ and $\tilde{\mathrm{b}} \equiv(p, q)+\mathrm{b}$. The same is true for $\left(\mathrm{d}^{\prime}\right) \mathrm{P}$ : for otherwise $\left(R_{I}\right) \mathrm{P}$ would straddle part of its own boundary on the line $\ell_{\lambda}^{(p-1, q)}$. Thus

$$
(\mathrm{ac}) \mathrm{P} \subset(\mathrm{a} \overline{\mathrm{d}}) \mathrm{P} \subset \mathrm{ab}
$$

and

$$
\left(\mathrm{b}^{\prime} \mathrm{d}^{\prime}\right) \mathrm{P} \subset \tilde{a} \tilde{b}
$$

So the parallelogram $\left(R_{I}\right) \mathrm{P}$ begins (as shown in the leftmost figure of Figure 31) with its left vertical boundary contained in the segment ab on the line $\ell_{\mu}$ and ends (as shown in the middle figure of Figure 31) with its right vertical boundary contained in the segment $\tilde{\mathrm{a}} \tilde{\mathrm{b}}$ on the line $\ell_{\mu}^{(p, q)}$. We see that the $\left(R_{I}\right) \mathrm{P}$ begins by striping across $R_{I}$. Its lower horizontal boundary lies on the line $\ell_{\lambda}$ and covers the lower horizontal boundary of $R_{I}$. Not shown in the figure is the manner by which $\left(R_{I}\right) \mathrm{P}$ stripes across the top of the fundamental region consisting of $R_{I}+(r, s-1)$ and $R_{I I}+(r, s-1)$ with its upper horizontal boundary contained in the line $\ell_{\lambda}^{\left(\mathrm{a}^{\prime \prime \prime}\right) \mathrm{P}}=$ $\ell_{\lambda}^{(r, s)}$. The parallelogram $\left(R_{I}\right) \mathrm{P}$ ends by striping through $R_{I I}+(p-1, q-1)$.

The other parallelogram $\left(R_{I I}\right) \mathrm{P}$ begins where $\left(R_{I}\right) \mathrm{P}$ leaves off. Its left vertical boundary is contained in $\tilde{a} \tilde{b}$, and its right one in $\tilde{\tilde{a}} \tilde{\tilde{b}}$ where $\tilde{\tilde{a}} \equiv(p+r, q+s)$ and $\tilde{\tilde{\mathrm{b}}} \equiv \mathrm{b}+(p, q)$, as shown in the middle figure of Figure 31 . The first set that $\left(R_{I I}\right) \mathrm{P}$ stripes through is $R_{I}+(p, q)$ and the last $R_{I I}+(p+r-1, q+s-1)$. It stripes through $R_{I}+(p, q)$ on top of $R_{I} \cap\left(R_{I}\right) \mathrm{P}+(p, q)$ and ends at the bottom of $R_{I I}+(p+r-1, q+s-1)$, as shown in Figure 31 .

As drawn in Figure 29, the line segment aa"' lies totally in $\overline{R_{I}}$ connecting its bottom horizontal boundary with its top: so $\left(R_{i}\right) \mathrm{P}$ has the property that it stripes across $R_{I}+(m, n)$ if and only if it passes through the interior of the line segment $\mathrm{aa}^{\prime \prime \prime}+(m, n)$. But one must bear in mind that, unless we have arranged for $\mathrm{c}^{\prime}$ to 
be located in the principal unit square, the line segment aa" $a^{\prime \prime \prime}$ may not lie totally in $\overline{R_{I}}$. Nevertheless, because of the way $\left(R_{I}\right) \mathrm{P}$ begins and ends it obeys this property when $(m, n)=(0,0)$ and $(m, n)=(p-1, q)$. Consequently, it also obeys this property for all intermediary lattice points. By intermediary we mean at $(m, n)$ where $0 \leq m \leq p-1$ and $0 \leq n \leq q$. Similarly for $\left(R_{I I}\right) \mathrm{P}$ it satisfies the property for $(m, n)=(p, q),(m, n)=(p+r-1, q+s-1)$, and thus for all intermediary $(m, n)$.

The number of times $\left(R_{i}\right) P$ passes through a lattice translate aa ${ }^{\prime \prime \prime}$ equals the number of different lines $x=$ integer crossed by $\left(R_{i}\right) P$, which equals $p$ for $\left(R_{I}\right) \mathrm{P}$ and $r$ for $\left(R_{I I}\right) \mathrm{P}$.

Similarly, a parallelogram $\left(R_{i}\right) \mathrm{P}$ intersects lattice translate of $R_{I I}$ if and only if $\left(R_{i}\right) \mathrm{P}$ crosses the same lattice translate of $\mathrm{a}^{\prime \prime \prime} \mathrm{a}^{\prime \prime}$, and the number of times this happens equals the number of lines $y=$ integer crossed by $\left(R_{i}\right) \mathrm{P}$, which equals $q$ for $\left(R_{I}\right) \mathrm{P}$ and $s$ for $\left(R_{I I}\right) \mathrm{P}$.

Remark. A consequence of the fact that the right contracting boundary of $\left(R_{I}\right) \mathrm{P}$ is contained in a lattice translate of ab is that $\phi R_{I} \cap R_{I I} \neq \emptyset$, which means $q>0$. But this we already know from the property that $P$ is hyperbolic. Similarly, $r>0$. However, either $p$ or $s$ could be 0 , but not both.

Step 5. The transition matrix associated with mapping of the Markov generator $\mathcal{R}^{*}$ by $\phi$ coincides with a matrix which specifies the automorphism-namely, $\mathrm{P}$ - the edge graph of which is illustrated in Figure 28.

From what we have established there are $N^{*}=p+q+r+s$ parallelograms $R_{i}^{*}$ in $\mathcal{R}^{*}$. We separate the subscripts into four sets:

$$
\begin{gathered}
\{1, \ldots, p\} \\
\{p+1, \ldots, p+q\} \\
\{p+q+1, \ldots, p+q+r\} \\
\{p+q+r+1, \ldots, p+q+r+s\} .
\end{gathered}
$$

We label the members of $\mathcal{R}^{*}$ accordingly:

$$
\begin{aligned}
R_{1}^{*} \cup \cdots \cup R_{p}^{*} & =R_{I} \cap \phi R_{I}, \\
R_{p+1}^{*} \cup \cdots \cup R_{p+q}^{*} & =R_{I I} \cap \phi R_{I}, \\
R_{p+q+1}^{*} \cup \cdots \cup R_{p+q+r}^{*} & =R_{I} \cap \phi R_{I I}, \\
R_{p+q+r+1}^{*} \cup \cdots \cup R_{p+q+r+s}^{*} & =R_{I I} \cap \phi R_{I I} .
\end{aligned}
$$

As we have said before: if $\phi h_{i}(\mathrm{p}) \cap R_{k}^{*} \neq \emptyset$ for one horizontal $h_{i}(\mathrm{p})$ of $R_{i}$, then $\phi h_{i}(\mathrm{q}) \cap R_{k}^{*} \neq \emptyset$ for any other horizontal $h_{i}$ (q) of $R_{i}$. Therefore, the image of each parallelogram $R_{k}^{*}$ contained in an $R_{i}$ intersects the same elements of $\mathcal{R}^{*}$. From this we get that $\phi R_{k}^{*} \cap R_{l}^{*} \neq \emptyset$, equivalently $R_{k}^{*} \cap \phi^{-1} R_{l}^{*} \neq \emptyset$, whenever either

$$
k \in(1) \cup(3) \text { and } l \in(1) \cup(2),
$$

or

$$
k \in(2) \cup(4) \text { and } l \in(3) \cup(4) .
$$

Case II: $\lambda>0, \mu<0$. 


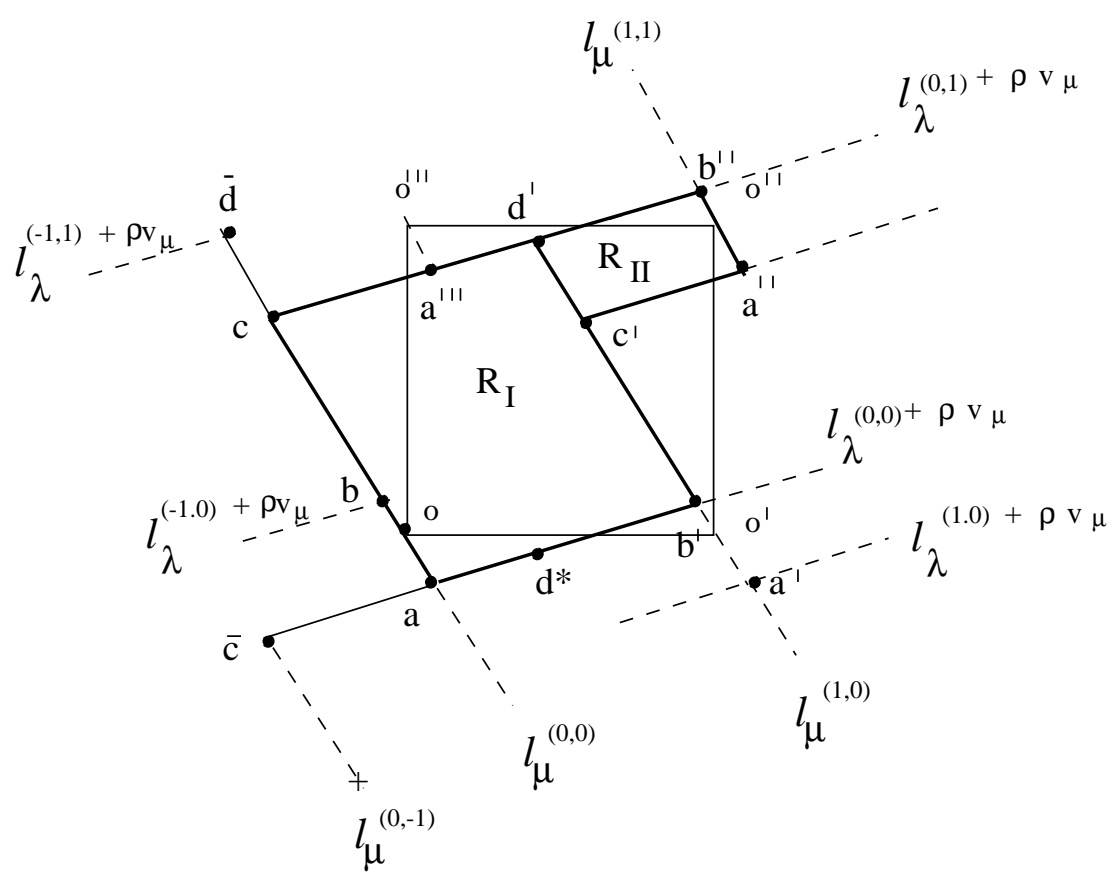

Figure 32(A). New principal Markov fundamental region, a translation of the previous one with o between $\mathrm{a}$ and $\mathrm{b}$

Except for the lattice points of the unit square labelled by o's we redefine the other ones in Figure 29 by translating them by $\rho \mathrm{v}_{\mu}$ with $\rho$, chosen so that

$$
(\overline{\mathrm{d}}) \mathrm{P}=\mathrm{a} \text {. }
$$

For example, $a_{\text {new }}=a_{\text {old }}+\rho \mathrm{v}_{\mu}$. Whereas before point a was the fixed point $\mathrm{o}$, now it is not. Figures $32(\mathrm{~A})$ and $32(\mathrm{~B})$ depict new principal Markov fundamental regions in which the old one of Figure 29 has been translated by $\rho \mathrm{v}_{\mu}$. The difference in the two figures is the location of o with respect to $\mathrm{b}$. In a moment we shall argue that Figure 32(B) cannot occur.

Step 1. The new partition $\mathcal{R}=\left\{R_{I}, R_{I I}\right\}$ is Markov.

The proof of this is the same as in Step 1 of Case I except the verification of

$$
\begin{aligned}
\phi \partial_{V} \mathcal{R} & \subset \partial \mathcal{R}_{V} \subset \partial \mathcal{R}, \\
\phi^{-1} \partial_{H} \mathcal{R} & \subset \partial_{H} \mathcal{R} \subset \partial \mathcal{R}
\end{aligned}
$$

is more involved and is as follows.

For this new partition, (8.6) remains valid: namely,

$$
\begin{gathered}
\partial_{H} \mathcal{R}=\overline{\mathrm{cb}^{\prime} \subset \ell_{\lambda}^{a},} \\
\partial_{V} \mathcal{R}=\mathrm{a} \overline{\mathrm{d}} \subset \ell_{\mu} .
\end{gathered}
$$

Before there was a common fixed point on $\partial_{H} \mathcal{R}$ and $\partial_{V} \mathcal{R}$ : namely, origin $=$ $\partial_{H} \mathcal{R} \cap \partial_{V} \mathcal{R}$, but now the intersection $\partial_{H} \mathcal{R} \cap \partial_{V} \mathcal{R}$ is no longer a fixed point. The line segment a $\bar{d}$ still contains the origin as a fixed point. The line segment $\bar{c} b$ also 


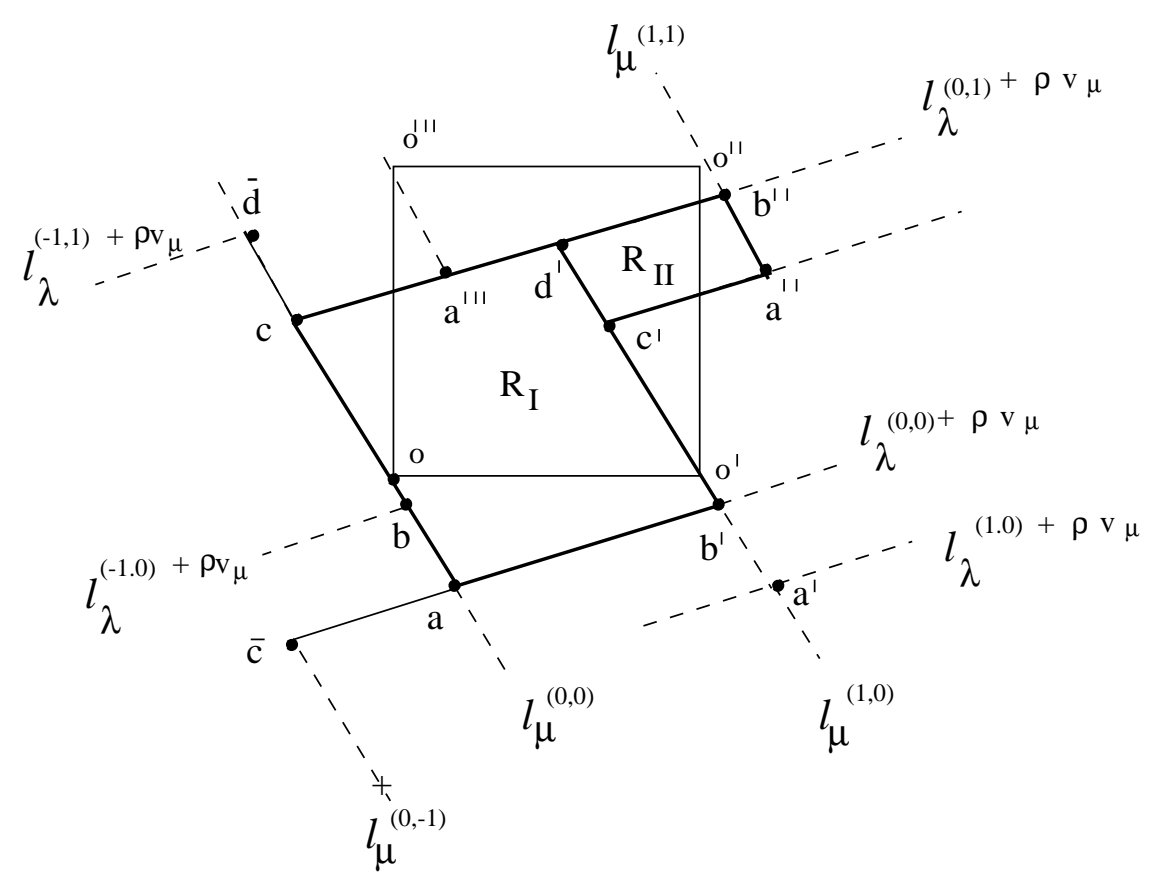

Figure 32(B). Hypothetical new principal Markov fundamental region, with o above $\mathrm{b}$

contains a fixed point, but this is not so obvious. This will be a consequence of the following version of (8.7):

$\left(\overline{\mathrm{c}} \mathrm{b}^{\prime}\right) \mathrm{P} \supset$ lattice translate of $\overline{\mathrm{cb}}{ }^{\prime}$

$(\mathrm{a} \overline{\mathrm{d}}) \mathrm{P} \subset \mathrm{a} \overline{\mathrm{d}}$.

Verification of the first relation is more difficult than before. That the second relation holds is immediate because the translation was chosen to make it so. The first relation rests on the property of the partition that a, b, c, d are the only points on the contracting boundary which have equivalent ones on the expanding. There are no others!

Recall $\mathrm{d}^{*}=\mathrm{d}^{\prime}-(0,1)$. Now because $(\overline{\mathrm{d}}) \mathrm{P}=\mathrm{a}$, the point $\hat{\mathrm{a}} \equiv\left(\mathrm{d}^{*}\right) \mathrm{P}$ is a lattice translate of a and the line $\ell_{\lambda}^{\mathrm{a}}$ is mapped to the line $\ell_{\lambda}^{\hat{a}}$ by P. See Figure 33. Let $\hat{\mathrm{c}}, \hat{\mathrm{b}}$, and $\hat{\mathrm{d}}$ be the points on the line $\ell_{\lambda}^{\hat{\mathrm{a}}}$ that are lattice translates of the points $\overline{\mathrm{c}}$, $\mathrm{b}^{\prime}$, and $\mathrm{d}^{*}$. We must show that $\left(\overline{\mathrm{c}} \mathrm{b}^{\prime}\right) \mathrm{P}$ contains $\hat{\mathrm{c}} \hat{\mathrm{b}}$. Because the point $\left(\mathrm{d}^{*}\right) \mathrm{P}$ lies on the line $\ell_{\lambda}^{\hat{a}}$, so do the points $(\overline{\mathrm{c}}) \mathrm{P}$ and $\left(\mathrm{b}^{\prime}\right) \mathrm{P}$. Since $(\mathrm{b}) \mathrm{P}$ and $(\mathrm{c}) \mathrm{P}$ are contained in the segment $\mathrm{a} \overline{\mathrm{d}}$ and the pair of points $\left(\mathrm{b}^{\prime}\right) \mathrm{P}$ and $(\overline{\mathrm{c}}) \mathrm{P}$ are lattice translates of neither a, b, c, nor $\bar{d}$, this pair lies outside the segment $\hat{b} \hat{c}$. Since $\left(\mathrm{d}^{*}\right) \mathrm{P}$ lies inside, the segment $\left(\bar{c} b^{\prime}\right) \mathrm{P}$ overlaps $\hat{c} \hat{b}$.

Now we are in a position to prove that Figure 32(B) cannot occur. Let $\hat{\hat{a}}=$ $(p, q)+\rho \mathrm{v}_{\mu}$. Since $(\overline{\mathrm{d}}) \mathrm{P}=\mathrm{a}$, we have that $\tilde{\mathrm{a}}=\mathrm{d}^{\prime} \mathrm{P}$. Let $\hat{\hat{\mathrm{b}}}$ be the point equivalent 


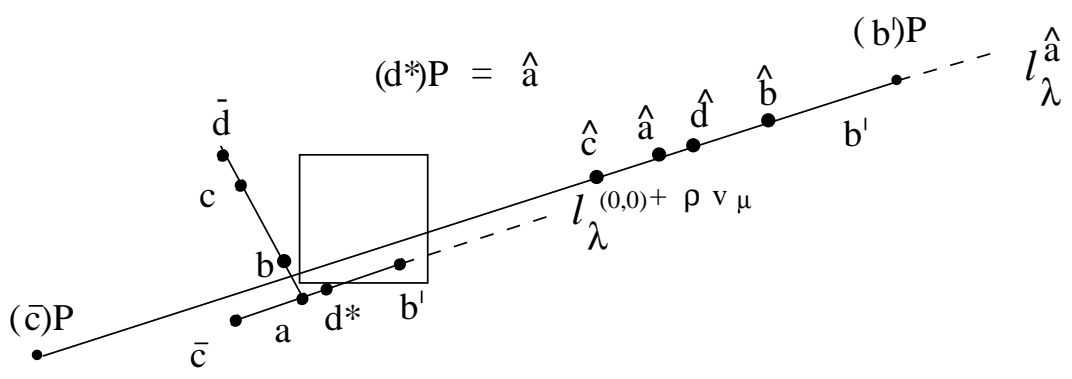

FiguRE 33. Image of expanding boundary of new principal Markov fundamental region

to $\mathrm{b}^{\prime}$ on the line $\ell_{\mu}^{(p, q)}$, upon which ã lies, and let $\hat{\mathrm{c}}$ be the lattice translate of $\bar{c}$ on the line $\ell_{\lambda}^{(p, q)+\rho v_{\mu}}$. The segment $\hat{\hat{c}} \hat{\hat{b}}$ is a lattice translate of the horizontal boundary of $\overline{R_{I}} \cup \overline{R_{I I}}$. If $\mathrm{b}$ were below o on the line $\ell_{\mu}^{(0,0)}$, then by virtue of the fact that $\mu$ is negative the point $\mathrm{b}^{\prime} \mathrm{P}$ would lie above $(p, q)$ on this line, and hence above $\hat{\mathrm{b}}$. Since $\mathrm{d}^{\prime} \mathrm{P}=\tilde{\mathrm{a}}$ lies below $\hat{\hat{b}}$, the parallelogram $R_{I} \mathrm{P}$ would straddle part of its own horizontal boundary: namely, the segment $\hat{\mathrm{c}} \hat{\mathrm{b}}$. This would contradict the fact that $R_{I} \mathrm{P}$ is a subset of a fundamental region.

Step 2. The family $\mathcal{R}^{*}=\left\{R_{k}^{*}: 1 \leq k \leq N^{*}\right\}$ consisting of all connected components of the sets $R_{i} \cap \phi^{-1} R_{j} \in \mathcal{R} \vee \phi^{-1} \mathcal{R}$ is a Markov partition.

Proof is the same as Step 2 of Case I.

Step 3. The Markov partition $\mathcal{R}^{*}$ is a generator.

Proof, same as Case I, Step 3.

Step 4. Let \# $\left(\phi R_{i} \cap R_{j}\right)$ denote the number of disjoint parallelograms in the intersection $\phi R_{i} \cap R_{j}$.

$$
\begin{aligned}
\#\left(\phi R_{i} \cap R_{I}\right) & \equiv \text { number of lines } x=0, x=1, \ldots, \text { traversed by }\left(R_{i}\right) P \\
\#\left(\phi R_{i} \cap R_{I I}\right) & \equiv \text { number of lines } y=1, y=2, \ldots, \text { traversed by }\left(R_{i}\right) P
\end{aligned}
$$

which leads to

$$
\begin{aligned}
\#\left(\phi R_{I} \cap R_{I}\right) & =x \text {-coordinate of }(0,1) \mathrm{P}=p ; \\
\#\left(\phi R_{I I} \cap R_{I}\right) & =x \text {-coordinate of }(1,1) \mathrm{P}-(1,0) \mathrm{P}=q ; \\
\#\left(\phi R_{I} \cap R_{I I}\right) & =y \text {-coordinate of }(0,1) \mathrm{P}=r ; \\
\#\left(\phi R_{I I} \cap R_{I I}\right) & =y \text {-coordinate of }(1,1) \mathrm{P}-(1,0) \mathrm{P}=s
\end{aligned}
$$

Argument similar to Case I, Step 4, but based on Figure 34. There is a slight difference here. The vertical line segment aa ${ }^{\prime \prime \prime}$ in Figure $32(\mathrm{~A})$ is offset from the line $x=0$, and $\mathrm{a}^{\prime \prime \prime} \mathrm{a}^{\prime \prime}$ does not lie on $y=1$. If $\rho v_{\mu}=(\xi, \eta)$, then

$$
\begin{aligned}
\#\left(\phi R_{i} \cap R_{I}\right) & \equiv \text { number of lines } x-\xi=0, x-\xi=1, \ldots, \text { traversed by }\left(R_{i}\right) P ; \\
\#\left(\phi R_{i} \cap R_{I I}\right) & \equiv \text { number of lines } y-\eta=1, y-\eta=2, \ldots, \text { traversed by }\left(R_{i}\right) P .
\end{aligned}
$$

To get the desired result we observe that the parallelogram $\left(R_{i}\right) P$ traverses the line $x-\xi=m$ if and only if it traverses the line $x=m$. Similarly the parallelogram traverses $y-\eta=m$ if and only if it traverses $y=m$. 


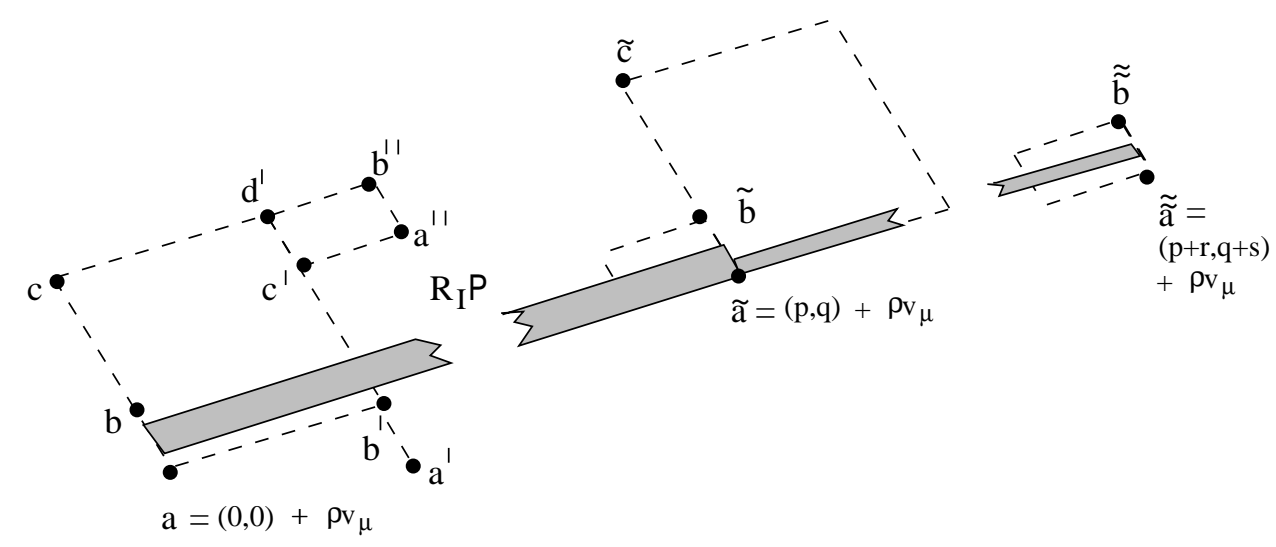

Figure 34 . How the new $\left(R_{I}\right) \mathrm{P}$ and $\left(R_{I I}\right) \mathrm{P}$ intersect various fundamental regions

Step 5. The transition matrix associated with mapping of the Markov generator $\mathcal{R}^{*}$ by $\phi$ coincides with the matrix which specifies the automorphism-namely, $\mathrm{P}-$ the edge graph of which is illustrated in Figure 28.

Argument same as Case I, Step 5.

Case III: $\lambda<0, \mu>0$.

The sets $R_{i}$ are the same as for Case I.

Step 1. The new partition $\mathcal{R}=\left\{R_{I}, R_{I I}\right\}$ is Markov.

This depends on establishing (8.6): namely,

$$
\begin{aligned}
\overline{\mathrm{c}} \mathrm{b}^{\prime} & \subset\left(\overline{\mathrm{c}} \mathrm{b}^{\prime}\right) P \\
(\mathrm{a} \overline{\mathrm{d}}) P & \subset \mathrm{a} \overline{\mathrm{d}} .
\end{aligned}
$$

The argument for the first relation is similar to that of Case II, Step 1. The argument for the second is the same as (8.7) of Case I.

Step 2. The family $\mathcal{R}^{*}=\left\{R_{k}^{*}: 1 \leq k \leq N^{*}\right\}$ consisting of all connected components of the sets $R_{i} \cap \phi^{-1} R_{j} \in \mathcal{R} \vee \phi^{-1} \mathcal{R}$ is a Markov partition.

Proof is the same as Step 2 of Case I.

Step 3. The Markov partition $\mathcal{R}^{*}$ is a generator.

Proof, same as Case I, Step 3.

Step 4. Let $\#\left(\phi R_{i} \cap R_{j}\right)$ denote the number of disjoint parallelograms in the intersection $\phi R_{i} \cap R_{j}$.

$$
\begin{aligned}
\#\left(\phi R_{i} \cap R_{I}\right) & \equiv \text { number of lines } x=0, x=-1, \ldots, \text { traversed by }\left(R_{i}\right) P \\
\#\left(\phi R_{i} \cap R_{I I}\right) & \equiv \text { number of lines } y=-1, y=-2, \ldots, \text { traversed by }\left(R_{i}\right) P,
\end{aligned}
$$

which leads to

$$
\begin{aligned}
\#\left(\phi R_{I} \cap R_{I}\right) & =x \text {-coordinate of }(0,1) \mathrm{P}=-p ; \\
\#\left(\phi R_{I I} \cap R_{I}\right) & =x \text {-coordinate of }(1,1) \mathrm{P}-(1,0) \mathrm{P}=-q ; \\
\#\left(\phi R_{I} \cap R_{I I}\right) & =y \text {-coordinate of }(0,1) \mathrm{P}=-r ; \\
\#\left(\phi R_{I I} \cap R_{I I}\right) & =y \text {-coordinate of }(1,1) \mathrm{P}-(1,0) \mathrm{P}=-s .
\end{aligned}
$$




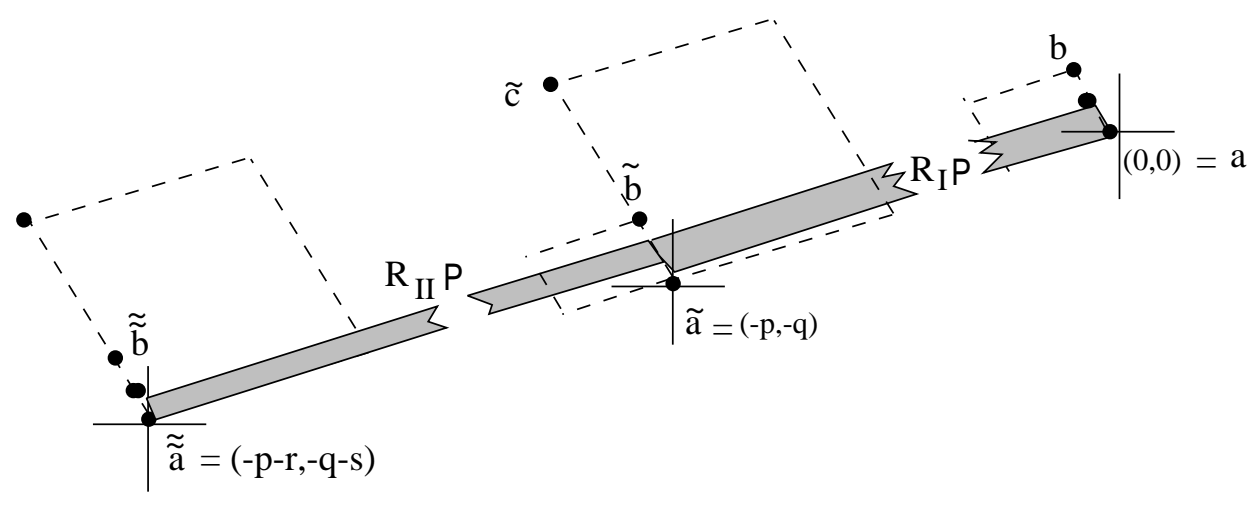

Figure 35. How $\left(R_{I}\right) \mathrm{P}$ and $\left(R_{I I}\right) \mathrm{P}$ intersect various fundamental regions for Case III

Argument the same as Case I, Step 4, but based on Figure 35.

Step 5. The transition matrix associated with mapping of the Markov generator $\mathcal{R}^{*}$ by $\phi$ coincides with matrix which specifies the automorphism-namely, $\mathrm{P}-$ the edge graph of which is illustrated in Figure 28.

Argument same as Case I, Step 5.

Case IV: $\lambda<0, \mu<0$.

The sets $R_{i}$ are the same as in Case II- i.e., translations of the ones of Case I.

Step 1. The new partition $\mathcal{R}=\left\{R_{I}, R_{I I}\right\}$ is Markov.

The argument is the same as in Case II, Step 1.

Step 2. The family $\mathcal{R}^{*}=\left\{R_{k}^{*}: 1 \leq k \leq N^{*}\right\}$ consisting of all connected components of the sets $R_{i} \cap \phi^{-1} R_{j} \in \mathcal{R} \vee \bar{\phi}^{-1} \overline{\mathcal{R}}$ is a Markov partition.

Proof same as Case I, Step 2.

Step 3. The Markov partition $\mathcal{R}^{*}$ is a generator.

Proof same as Case I, Step 3.

Step 4. Let \# $\left(\phi R_{i} \cap R_{j}\right)$ denote the number of disjoint parallelograms in the intersection $\phi R_{i} \cap R_{j}$.

$$
\begin{aligned}
\#\left(\phi R_{i} \cap R_{I}\right) & \equiv \text { number of lines } x=0, x=-1, \ldots, \text { traversed by }\left(R_{i}\right) P ; \\
\#\left(\phi R_{i} \cap R_{I I}\right) & \equiv \text { number of lines } y=-1, y=-2, \ldots, \text { traversed by }\left(R_{i}\right) P,
\end{aligned}
$$

which leads to

$$
\begin{aligned}
\#\left(\phi R_{I} \cap R_{I}\right) & =x \text {-coordinate of }(0,1) \mathrm{P}=-p ; \\
\#\left(\phi R_{I I} \cap R_{I}\right) & =x \text {-coordinate of }(1,1) \mathrm{P}-(1,0) \mathrm{P}=-q ; \\
\#\left(\phi R_{I} \cap R_{I I}\right) & =y \text {-coordinate of }(0,1) \mathrm{P}=-r ; \\
\#\left(\phi R_{I I} \cap R_{I I}\right) & =y \text {-coordinate of }(1,1) \mathrm{P}-(1,0) \mathrm{P}=-s .
\end{aligned}
$$

Argument the same as Case II, Step 4, but based on Figure 36.

Step 5. The transition matrix associated with mapping of the Markov generator $\mathcal{R}^{*}$ by $\phi$ coincides with matrix which specifies the automorphism-namely, $\mathrm{P}-$ the edge graph of which is illustrated in Figure 28. 


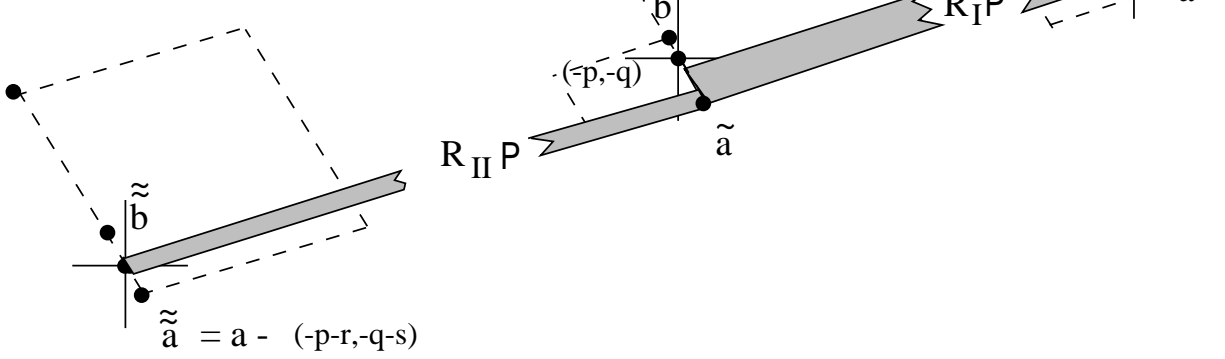

Figure 36 . How $\left(R_{I}\right) \mathrm{P}$ and $\left(R_{I I}\right) \mathrm{P}$ intersect various fundamental regions for Case IV

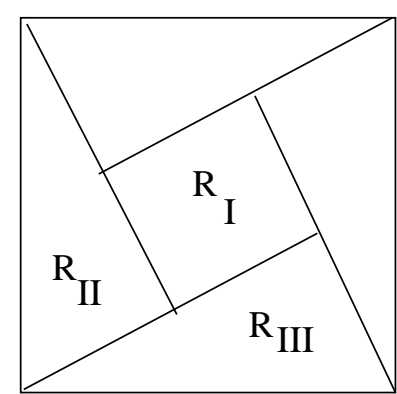

Figure 37. Partition from the "behold" proof of the Pythagorean theorem

Argument same as Case I, Step 5.

\section{Exercises.}

8.1 Let $(X, \phi)$ be the dynamical system and $\mathcal{R}=\left\{R_{i}: i=1,2,3\right\}$ the Markov partition appearing in Example 3.3. Show the partition $\mathcal{S}=\left\{S_{i}: i=0,1\right\}$, where $S_{0}=R_{1} \cup R_{2}$ and $S_{1}=R_{3}$ is Markov. Hint: $\mathcal{S}^{(2)}=\mathcal{R}$. Observe that associated directed graph $G$ is the vertex-labelled graph of Figure 2.

8.2 What is the maximum number of pre-images of $\pi$ in the last example? In Theorem 8.4?

8.3 Let $\phi$ be an automorphism of the $2 n$-torus given by a matrix $A$ such that $A^{m}$ is conjugate over $\mathbb{Q}$ to a block diagonal matrix, the blocks being $2 \times 2$ hyperbolic matrices. Construct a Markov partition for $\phi$.

8.4 Let $X=\mathbb{R}^{2} / \mathbb{Z}^{2}$ be the 2-dimensional torus, $\phi$ be the automorphism given by the matrix

$$
\mathrm{A}=\left(\begin{array}{ll}
1 & 1 \\
1 & 0
\end{array}\right)
$$

and $\mathcal{R}=\left\{R_{I}, R_{I I}, R_{I I I}\right\}$ the partition given in Figure 37. Consider the equivalence relation $(x, y) \sim\left(x \prime, y^{\prime}\right)$ when $(x, y)=(-x,-y)$. Let $\widetilde{X}=X / \sim$ be the space of equivalence classes, $\widetilde{\phi}$ the induced mapping, and $\widetilde{R}$ the induced 
partition. Show that $\widetilde{R}$ is a topological Markov generator, the map $\pi$ defined by (5.11) satisfies $4.6(\mathrm{i})-(\mathrm{v})$, and $\widetilde{\phi}$ is not expansive.

\section{EPILOGUe}

The main theorem of Section 6 and its converse exhibit a duality between factor maps and Markov partitions. We are thus presented with a type of "chicken verses egg" question: which are more fundamental for getting concrete symbolic representations for concrete dynamical systems, Markov partitions or factor maps? In Example 3.2, we have with equal ease defined a factor map arithmetically, producing from it a Markov partition, and constructed a Markov partition, thereby obtaining a factor map. However, in Section 8, we only constructed a Markov partition for an arbitrary hyperbolic automorphism of the two-dimensional torus. In trying to repeat the success of dimension two by drawing Markov partitions for hyperbolic toral automorphisms in dimension three, one is doomed to failure as Bowen proves in [Bo4]. He shows that at no point on the contracting boundary of a member of a Markov partition can there exist a tangent plane. The boundary is forced to be a fractal. Even if such a figure could be constructed, rendering is certainly difficult, let alone incorporating it in a tiling of three space in a manner suitable for viewing. And then what about four dimensions and higher? E. Cawley has shown $[\mathrm{C}]$ that smooth Markov partitions for automorphisms of n-dimensional tori can only occur in even dimensions and only for automorphisms related in a special way to the Cartesian product of hyperbolic automorphisms of 2 -tori. She proves that if an automorphism $\phi$ given by an $n \times n$ hyperbolic matrix $A$ has a smooth Markov partition, then $n$ is even and there exists an integer $m$ such that $A^{m}$ is conjugate over $\mathbb{Q}$ to a block diagonal matrix where each block is a $2 \times 2$ hyperbolic matrix (See Exercise 8.3). However, for most automorphisms in dimensions other than two an arithmetic method seems the only hope. Kenyon, Vershik [KV], and Praggastis [Pr1], [Pr2] attack the problem in this manner and obtain factor maps arithmetically for hyperbolic toral automorphisms.

Ultimately the answer to our chicken-egg question will probably turn out to be that neither takes precedence over the other. Bowen's proof of existence of Markov partitions and hence finite factor maps for general hyperbolic axiom A diffeomorphisms supports this point of view [Bo3]. In this work Bowen uses an argument employing a combination of both methods and bootstraps his way to the desired result. It starts with a cover whose members have small diameter with respect to the expansive constant. A topological Markov shift is constructed by using the labels of the members as symbols and defining transitions according to the rule that the $j$-th symbol follows the $i$-th if the image of the $i$-th member of the cover intersects the $j$-th. Using stable and unstable manifolds, one is able to define a factor map from the symbolic shift to the phase space. This symbolic extension is much too big. The cardinality of pre-images under this map can be non-denumerable. However, the images of cylinder sets form a second cover whose members are abstract rectangles obeying a Markovian property. This cover though may fail to form a topological partition for two reasons: some members may have no interior, and pairs of them may overlap in more than just boundary points. This cover is pared down by eliminating those sets with no interior. The overlap problem is solved by using some geometry of abstract rectangles: namely, two abstract rectangles overlapping in an open set can be partitioned into non-overlapping abstract rectangles. The last step 
in getting a topological partition is to partition the members of this last cover by abstract rectangles, no pair of which overlaps in an open set. Through all of this Markov property is still maintained by the members of this final partition. That this is a generator is gotten by the same argument as Proposition 5.8.

Returning to the line of investigation of Kenyon, Vershik, and Praggastis, there remain things to be understood, such as the connectivity of cylinder set images under arithmetically defined factor maps. Furthermore, the general case of Markov partitions for hyperbolic automorphisms of n-dimensional tori has not yet been treated.

\section{REFERENCES}

[ATW] Roy Adler, Charles Tresser, Patrick A. Worfolk, Topological conjugacy of linear endomorphisms of the 2-torus, Trans. Amer. Math. Soc. 349 (1997), 1633-1652. MR 97m:58181

[AW1] R.L. Adler and B. Weiss, Entropy, a complete metric invariant for automorphisms of the torus, Proc. Natl. Acad. Sci. 57 (1967), 1573-1576. MR 35:3031

[AW2] Roy L. Adler and Benjamin Weiss, Similarity of automorphisms of the torus, Memoirs American Math. Soc. 98 (1970). MR 41:1966

[Be] K. Berg, On the conjugacy problem for K-systems, Ph.D. Thesis, University of Minnesota, 1967.

[Bo1] R. Bowen, Markov partitions for Axiom A diffeomorphisms, Amer. J. Math. 92 (1970), 725-747. MR 43:2740

[Bo2] On Axiom A Diffeomorphisms, Regional Conference Series in Mathematics, No. 35, American Mathematical Society, Providence, Rhode Island, 1978. MR 58:2888

[Bo3] Equilibrium States and the Ergodic Theory of Anosov Diffeomorphisms, Lecture Notes in Mathematics, Vol. 470, Springer-Verlag, Berlin, Heidelberg, New York, 1975. MR 56:1364

[Bo4] , Markov partitions are not smooth, Proc. Amer. Math. Soc. 71 (1970), 130-132. MR 57:14055

[C] E. Cawley, Smooth Markov partitions and toral automorphisms, Ergodic Th. and Dynam. Sys. 11 (1991), 633-651. MR 92k:58199

[FS] A. Fathi and M. Shub, Some dynamics of pseudo-Anosov diffeomorphisms, Astérique 66-67 (1979), 181-207.

[F] D. Fried, Finitely presented dynamical systems, Ergodic Th. and Dynam Sys. 7 (1987), 489-507. MR 89h:58157

[H] J. Hadamard, Les surfaces á courbures opposées et leurs lignes geodésiques, Journal de Mathematiques. 5 série IV (1898), 27-73.

[HK] B. Hasselblatt and A. Katok, Introduction to the Modern Theory of Dynamical Systems, Cambridge University Press, Cambridge, New York, Melbourne, 1995. MR 96c:58055

[KV] R. Kenyon and A. Vershik, Arithmetic construction of sofic partitions of hyperbolic toral automorphisms, Prepublication ou Rapport de Recherce no. 178, Ecole Normale Superieure de Lyon, 1995.

[LM] D. Lind and B Marcus, An Introduction to Symbolic Dynamics and Coding, Cambridge University Press, Cambridge, New York, Melbourne, 1995. MR 97a:58050

[Pr1] B. Praggastis, Markov partitions for hyperbolic toral automorphisms, Ph.D. Thesis, University of Washington, 1994.

[Pr2] Numeration systems and Markov partitions from self-similar tilings, Trans. Amer. Math. Soc. (to appear).

[S] M. Shub, Global Stability of Dynamical Systems, Springer-Verlag, New York, Berlin, Heidelberg, London, Paris, Tokyo, 1987. MR 87m:58086

[Si1] Ya. G. Sinai, Markov partitions and c-diffeomorphisms, Functional Analysis and Its Applications 2 (1968), 61-82. MR 38:1361

[Si2] _ Construction of Markov partitions, Functional Analysis and Its Applications 2 (1968), 245-253. MR 40:3591

[Sm] S. Smale, Differentiable dynamical systems, Bull. Amer. Math. Soc. 73 (1967), 747-817. MR 37:3598 
ROY L. ADLER

[T] W. P. Thurston, On the geometry and dynamics of diffeomorphisms of surfaces, Bull. Amer. Math. Soc. (N.S.) 19 (1988), 417-431. MR 89k:57023

[W] R. F. Williams, The "DA" maps of Smale and structural stability, Proc. Symp. in Pure Math., vol. 14, Amer. Math. Soc., Providence, RI, 1970, pp. 329-334. MR 41:9296

Mathematical Sciences Department, iBM, Thomas J. Watson Research Center, YorkTOWn Heights, New York 10598

E-mail address: adler@watson.ibm.com 